University of Tennessee Health Science Center

UTHSC Digital Commons

$12-2008$

\title{
Controlled Release of Insulin and Modified Insulin from a Novel Injectable Biodegradable Gel
}

\author{
Om Anand \\ University of Tennessee Health Science Center
}

Follow this and additional works at: https://dc.uthsc.edu/dissertations

Part of the Pharmaceutics and Drug Design Commons

\section{Recommended Citation}

Anand, Om , "Controlled Release of Insulin and Modified Insulin from a Novel Injectable Biodegradable Gel" (2008). Theses and Dissertations (ETD). Paper 16. http://dx.doi.org/10.21007/etd.cghs.2008.0013.

This Dissertation is brought to you for free and open access by the College of Graduate Health Sciences at UTHSC Digital Commons. It has been accepted for inclusion in Theses and Dissertations (ETD) by an authorized administrator of UTHSC Digital Commons. For more information, please contact jwelch30@uthsc.edu. 


\title{
Controlled Release of Insulin and Modified Insulin from a Novel Injectable Biodegradable Gel
}

\author{
Abstract \\ The objective of the study was to develop a controlled release dosage form of \\ insulin, which can provide basal concentrations of insulin in diabetic rats for 1 to 2 weeks after a single \\ subcutaneous injection. \\ A biodegradable injectable drug delivery gel was prepared by dissolving a \\ biodegradable polymer, polylactic-co-glycolic acid (PLGA), in biocompatible \\ plasticizer(s), triethyl citrate (TEC) and/or acetyl triethyl citrate (ATEC). Insulin was \\ then loaded into the blank gel to form an insulin suspension in the polymer solution. \\ After the insulin-loaded gel was injected subcutaneously, the plasticizer(s) dissolved in the aqueous \\ media and were gradually taken away from the gel. The polymer precipitated after the plasticizer(s) were \\ extracted by the aqueous medium and a solid depot of insulin was formed. The insulin was released \\ slowly from the depot by a combination of drug diffusion and erosion of the polymer.
}

In the first part of this study, the effect of different water-soluble and waterinsoluble zinc salts on blood glucose lowering effect of insulin in type-2 diabetic ZDF rats was investigated. Insulin formulations containing varying concentrations of different water-soluble and waterinsoluble zinc salts were prepared and injected subcutaneously in type-2 ZDF rats and blood glucose concentration lowering effect was studied. Insulin in presence of water-soluble salts of zinc could suppress blood glucose concentrations in ZDF rats for up to 16 hours.

Insulin was loaded into different gel formulations (5\% PLGA (i.v. 0.09, acid end group), ATEC:TEC (3:1) and 4\% insulin) and tested in vivo. However, these insulin- loaded gel formulations only suppressed the blood glucose concentrations in the ZDF rats for 1 day after a single subcutaneous injection. In order to achieve longer control over the release of insulin from the gels, a water-soluble salt, zinc sulfate was incorporated in these insulin containing gels at different concentrations. A biodegradable injectable gel formulation prepared with zinc sulfate was able to maintain low blood glucose concentrations for up to 8 to 10 days following a single subcutaneous injection.

In order to achieve better glucose control after the release of insulin from the gels, insulin glargine particles were purified from commercially available Lantus ${ }^{\circledR}$ formulation. The freeze dried insulin glargine particles were then loaded into the blank gels and tested in vivo. The formulation prepared with 5\% PLGA (i.v. 0.09, acid end group), ATEC:TEC (3:1) and 4\% insulin glargine was able to suppress the blood glucose concentrations of the ZDF rats significantly for 10 days after a single subcutaneous injection. The concentration of insulin glargine was maintained between $260 \pm 134.9 \mathrm{mIU} / \mathrm{L}$ and $188 \pm 55.9 \mathrm{mIU} / \mathrm{L}$ until day 10 after single subcutaneous injection. The addition of zinc sulfate to the formulations prepared with purified insulin glargine particles further slowed down the drop in blood glucose concentrations.

\section{Document Type}

Dissertation

\section{Degree Name}

Doctor of Philosophy (PhD)

\section{Program}

Pharmaceutical Sciences 
Research Advisor

Atul J. Shukla, Ph.D.

\section{Keywords}

Controlled release, insulin, modified insulin, glargine, biodegradable gel

\section{Subject Categories}

Pharmaceutics and Drug Design | Pharmacy and Pharmaceutical Sciences 


\title{
CONTROLLED RELEASE OF INSULIN AND MODIFIED INSULIN FROM A NOVEL INJECTABLE BIODEGRADABLE GEL
}

\author{
A Dissertation \\ Presented for \\ The Graduate Studies Council \\ The University of Tennessee \\ Health Science Center
}

\author{
In Partial Fulfillment \\ Of the Requirements for the Degree \\ Doctor of Philosophy \\ From The University of Tennessee
}

\author{
By \\ Om Anand \\ December 2008
}


Copyright (C) 2008 Om Anand All rights reserved 


\section{DEDICATION}

This dissertation is dedicated to my parents, Mr. Naresh Chandra and Mrs. Roshni Devi and my wife Mrs. Geetanjali Dabas and my son Ishan, for their endless love and support. 


\section{ACKNOWLEDGEMENTS}

My sincerest gratitude goes to my major professor Dr. Atul J. Shukla, the finest advisor in this world, for the opportunities, valuable advice, motivation, guidance and support given to me during the course of this research work.

I would like to thank my other committee members, Dr. James R. Johnson, Dr. Abbas E. Kitabchi, Dr. Mitchell S. Steiner and Dr. Bernd Meibhom for their suggestions, advice and help.

I feel so lucky that I have been working with a nice and helpful group during the past five years, Dr. Yichun Sun, Dr. Yingxu Peng, Dr. Wen Qu, Dr. Quanmin Chen, Dr. Paras Jain, Dr. WeiQiang Cheng, Dr. Bo Jiang, Dr. Shipeng Yu, Dr. Maria Gerald Rajan, Dr. Chaoju Xio, Namrata, Sonia, Yinqi Zhou and Suresh, I am really grateful to the group and they have made my study and research at UT an enjoyable one.

My special thanks go to Dr. Quanmin Chen, who did extensive basic work to establish this project and taught me different techniques to be useful in the project project. I acknowledge his help and guidance throughout the projrct.

I would like to thank Dr. Timothy Mandrell for his help in animal work and Dr. Syamal K. Bhattacharya for his help in determination of zinc concentrations.

I would like to thank the University of Tennessee Health Science Center for providing the opportunity and financial assistance to pursue a Doctor of Philosophy degree, and all the faculty and staff in Department of Pharmaceutical Sciences for their help and support during my graduate study.

I would like to thank Suresh Potharaju, whose help enabled me to complete the formatting part of this work.

Nothing in words could have expressed my deepest appreciation to my parents, Mr. Naresh Chandra and Mrs. Roshni Devi, my wife Mrs. Geetanjali Dabas, my elder brother, Dinesh, my parent-in-laws, Dr. S.S. Dabas and Mrs. Santosh Dabas, my sister-in-law Sonal and brother-in-law Sunny, and my friend Harpreet Singh for their love and encouragement. I am here because of them. 


\begin{abstract}
The objective of the study was to develop a controlled release dosage form of insulin, which can provide basal concentrations of insulin in diabetic rats for 1 to 2 weeks after a single subcutaneous injection.

A biodegradable injectable drug delivery gel was prepared by dissolving a biodegradable polymer, polylactic-co-glycolic acid (PLGA), in biocompatible plasticizer(s), triethyl citrate (TEC) and/or acetyl triethyl citrate (ATEC). Insulin was then loaded into the blank gel to form an insulin suspension in the polymer solution. After the insulin-loaded gel was injected subcutaneously, the plasticizer(s) dissolved in the aqueous media and were gradually taken away from the gel. The polymer precipitated after the plasticizer(s) were extracted by the aqueous medium and a solid depot of insulin was formed. The insulin was released slowly from the depot by a combination of drug diffusion and erosion of the polymer.

In the first part of this study, the effect of different water-soluble and waterinsoluble zinc salts on blood glucose lowering effect of insulin in type-2 diabetic ZDF rats was investigated. Insulin formulations containing varying concentrations of different water-soluble and water-insoluble zinc salts were prepared and injected subcutaneously in type-2 ZDF rats and blood glucose concentration lowering effect was studied. Insulin in presence of water-soluble salts of zinc could suppress blood glucose concentrations in ZDF rats for up to 16 hours.
\end{abstract}

Insulin was loaded into different gel formulations (5\% PLGA (i.v. 0.09, acid end group), ATEC:TEC (3:1) and 4\% insulin) and tested in vivo. However, these insulinloaded gel formulations only suppressed the blood glucose concentrations in the ZDF rats for 1 day after a single subcutaneous injection. In order to achieve longer control over the release of insulin from the gels, a water-soluble salt, zinc sulfate was incorporated in these insulin containing gels at different concentrations. A biodegradable injectable gel formulation prepared with zinc sulfate was able to maintain low blood glucose concentrations for up to 8 to 10 days following a single subcutaneous injection.

In order to achieve better glucose control after the release of insulin from the gels, insulin glargine particles were purified from commercially available Lantus ${ }^{\circledR}$ formulation. The freeze dried insulin glargine particles were then loaded into the blank gels and tested in vivo. The formulation prepared with 5\% PLGA (i.v. 0.09, acid end group), ATEC:TEC (3:1) and $4 \%$ insulin glargine was able to suppress the blood glucose concentrations of the ZDF rats significantly for 10 days after a single subcutaneous injection. The concentration of insulin glargine was maintained between $260 \pm 134.9 \mathrm{mIU} / \mathrm{L}$ and $188 \pm$ $55.9 \mathrm{mIU} / \mathrm{L}$ until day 10 after single subcutaneous injection. The addition of zinc sulfate to the formulations prepared with purified insulin glargine particles further slowed down the drop in blood glucose concentrations. 


\section{TABLE OF CONTENTS}

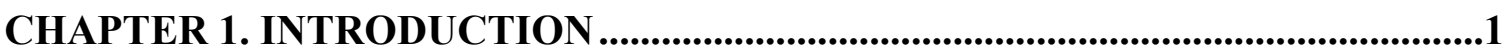

$1.1 \quad$ Diabetes mellitus ............................................................................... 1

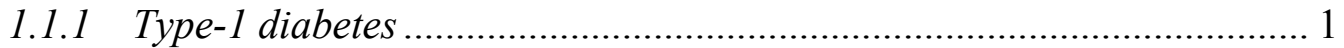

1.1.2 Type-2 diabetes ...................................................................... 2

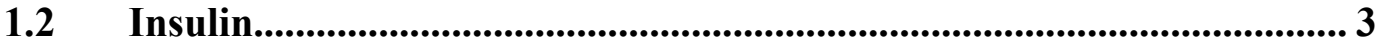

1.2.1 The structure of human insulin ………………….............................. 4

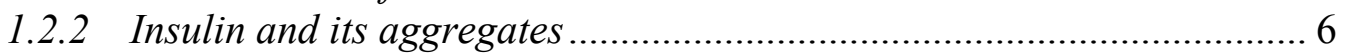

1.2.3 Insulin zinc and different state .......................................................... 7

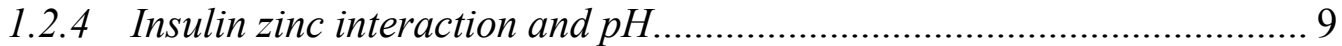

1.2.5 Insulin mechanism of action and degradation...................................... 9

$1.3 \quad$ Treatment of diabetes ............................................................................ 10

$1.4 \quad$ Non-invasive insulin delivery systems...................................................... 14

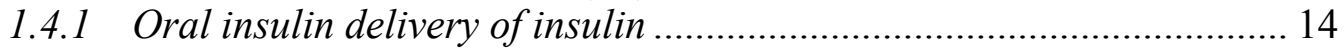

1.4.2 Nasal delivery of insulin .................................................................. 17

1.4.3 Pulmonary-lung delivery of insulin ................................................. 18

1.4.4 Ocular delivery of insulin .............................................................. 18

1.4.5 Rectal delivery of insulin ................................................................. 19

1.5 Controlled release of insulin ...................................................................... 19

1.5.1 Artificial pancreas ......................................................................... 19

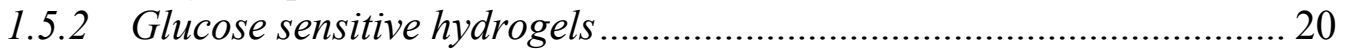

1.5.3 Extended release of insulin ............................................................... 20

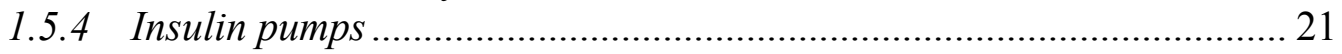

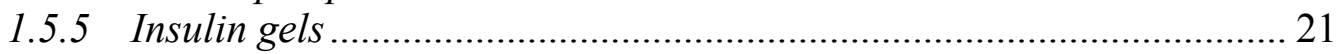

\section{CHAPTER 2. PROLONGED EFFECT OF INSULIN IN PRESENCE OF} WATER SOLUBLE AND WATER INSOLUBLE SOLUBLE ZINC SALTS..........23

$2.1 \quad$ Introduction................................................................................................. 23

2.2 Materials and methods ................................................................................ 24

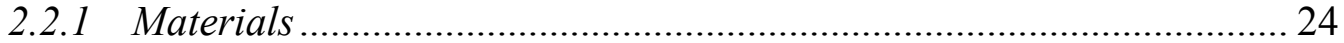

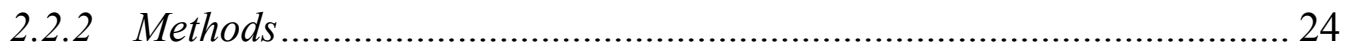

2.3 Results and discussion ................................................................................... 31

2.3.1 Pharmacodynamic studies of insulin in ZDF rats ............................. 31

2.3.2 In vitro release of insulin from insulin formulations with varying concentrations of zinc sulfate............................................................. 38

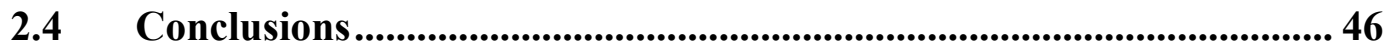

CHAPTER 3. CONTROLLED RELEASE OF INSULIN FROM NOVEL BIODEGRADABLE INJECTABLE GELS..................................................................49

3.1 Introduction ....................................................................................................... 49 
3.2 Materials and methods ............................................................................5 50

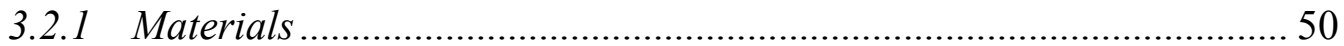

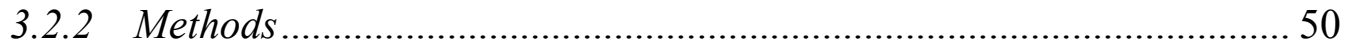

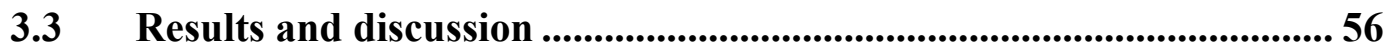

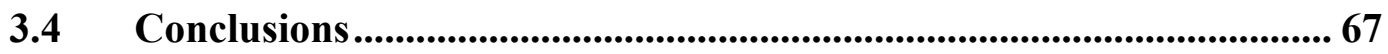

\section{CHAPTER 4. CONTROLLED RELEASE OF MODIFIED INSULIN}

GLARGINE FROM NOVEL BIODEGRADABLE INJECTABLE GELS ...............70

$4.1 \quad$ Introduction.................................................................................... 70

4.2 Materials and methods ....................................................................... 71

4.2.1 Materials ................................................................................... 71

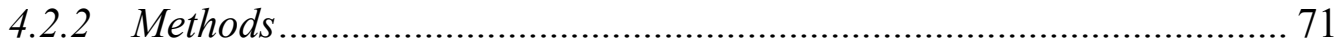

4.3 Results and discussion ................................................................................ 76

4.3.1 Purity of insulin glargine particles ...................................................... 76

4.3.2 Pharmacodynamic studies of insulin formulations in ZDF rats........... 79

4.3.3 In vivo studies of gel formulations loaded with insulin glargine

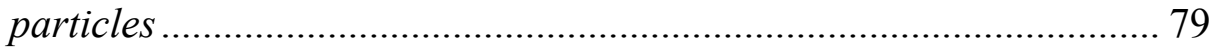

4.4 Conclusions ........................................................................................................ 95

LIST OF REFERENCES...................................................................................................97

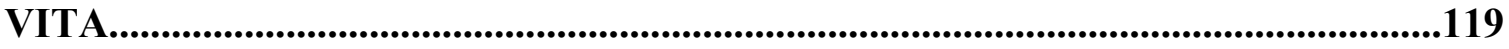




\section{LIST OF TABLES}

Table 1-1 Insulin and modified insulin preparations..............................................11

Table 2-1 Composition of insulin formulations containing zinc sulfate ......................25

Table 2-2 Composition of insulin formulations containing zinc chloride.....................26

Table 2-3 Composition of insulin formulations containing zinc carbonate ..................27

Table 2-4 Composition of insulin formulations containing zinc oxide .......................28

Table 3-1 Formulations of insulin suspensions …...........................................51

Table 3-2 Formulation composition of PLGA gel containing insulin and zinc .............52

Table 3-3 Formulation composition of PLGA gel containing insulin and zinc sulfate

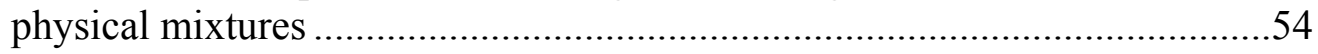

Table 3-4 Zinc contents in insulin-zinc co-precipitates ........................................55

Table 3-5 Formulation composition of PLGA gel of insulin ...................................57

Table 4-1 Composition of gel formulations used for in vivo studies ..........................74 


\section{LIST OF FIGURES}

Figure 1-1 Structure of human insulin ....................................................................

Figure 2-1 Blood glucose concentrations of ZDF rats after a single subcutaneous injection of insulin at a dose of $10 \mathrm{IU} / \mathrm{kg}($ Mean $\pm \mathrm{SEM}, \mathrm{n}=6)$

Figure 2-2 Comparison of blood glucose concentrations in ZDF rats after a single subcutaneous injection of insulin with varying concentrations of zinc sulfate (Insulin dose-10 U/kg) (Mean $\pm \mathrm{SEM}, \mathrm{n}=4)$....

Figure 2-3 Comparison of blood glucose concentrations in ZDF rats after a single subcutaneous injection of insulin with varying concentrations of zinc chloride (Insulin dose-10 U/kg) (Mean \pm SEM, $\mathrm{n}=4$ ).

Figure 2-4 Comparison of blood glucose concentrations in ZDF rats after subcutaneous injection of insulin, ultralente and insulin with varying concentrations of zinc carbonate (Insulin dose-10 U/kg) (Mean $\pm \mathrm{SEM}, \mathrm{n}$ $=4)$

Figure 2-5 Comparison of blood glucose concentrations in ZDF rats after a single subcutaneous injection of insulin with varying concentrations of zinc oxide (Insulin dose-10 U/kg) (Mean \pm SEM, $\mathrm{n}=4$ ).

Figure 2-6 Comparison of blood glucose concentrations in ZDF rats after a single subcutaneous injection of insulin with water-soluble and water-insoluble zinc salts (Insulin dose-10 U/kg) (Mean $\pm \mathrm{SEM}, \mathrm{n}=4)$

Figure 2-7 Comparision of in vitro insulin release from insulin suspension formulations with and without zinc sulfate

Figure 2-8 Comparision of in vitro insulin release from insulin suspension formulations with zinc sulfate.

Figure 2-9 Percentage of insulin remaining in the dialysis bags after completion of in vitro release study of insulin formulations with varying concentrations of zinc

Figure 2-10 Concentrations of insulin in the supernatant obtained from formulations of insulin alone or insulin with zinc sulfate $(0.24 \mathrm{mg} / \mathrm{mL})$ at different $\mathrm{pH}$ values

Figure 2-11 $\mathrm{pH}$ and concentration of insulin in the supernatant obtained from formulations of insulin (RHI) with varying concentrations zinc sulfate 
Figure 2-12 Concentration of insulin (RHI) in the supernatant obtained from formulations of insulin alone at different $\mathrm{pH}$, or formulations of insulin with varying concentrations zinc sulfate......

Figure 2-13 Possible mechanism of extended in vivo effect of formulations of insulin with varying concentrations zinc sulfate.

Figure 3-1 Changes in blood glucose concentrations of ZDF rats after a subcutaneous injection of PLGA (5\% and 10\%) gel formulations loaded with 4\% insulin (Formulations OA-3-1 and OA-3-2) (Mean \pm SEM, $n=$ 5)

Figure 3-2 Changes in body weights of ZDF rats after subcutaneous injections of PLGA (5 and 10\%) gel formulations loaded with 4\% insulin (Formulations OA-3-1 and OA-3-2) (Mean \pm SEM, $n=5)$

Figure 3-3 Changes in blood glucose concentrations of ZDF rats after subcutaneous injections of PLGA (5\%) gel formulations loaded with 4\% insulin alone (Formulation OA-3-1) or insulin-zinc co-precipitates (Formulations OA3-7 and OA-3-8) (Mean $\pm \mathrm{SEM}, \mathrm{n}=5)$

Figure 3-4 Changes in blood glucose concentrations of ZDF rats after subcutaneous injections of PLGA (5\%) gel formulations loaded with 4\% insulin alone (Formulation OA-3-1) or insulin-zinc co-precipitates (Formulations OA3-9 and OA-3-10) (Mean \pm SEM, $n=5)$.

Figure 3-5 Changes in body weights of ZDF rats after subcutaneous injections of PLGA (5\%) gel formulations loaded with 4\% insulin-zinc co-precipitates (Formulations OA-3-7, OA-3-8, OA-3-9 and OA-3-10) (Mean \pm SEM, $n$ $=5)$

Figure 3-6 Changes in blood glucose concentrations of ZDF rats after subcutaneous injections of insulin alone (Formulations OA-3-1) or physical mixtures of insulin (4\%) and zinc sulfate (1 or 2\%) loaded in PLGA (5\%) gel formulations (Formulations OA-3-11 and OA-3-12) (Mean \pm SEM, $\mathrm{n}=$ 5)

Figure 3-7 Changes in blood glucose concentrations of ZDF rats after subcutaneous injections of insulin loaded in PLGA (5\%) gel formulations with different concentrations of zinc sulfate $(2.8 \%$ and $5.4 \%)$ (Formulations OA-3-13 and OA-3-14) (Mean \pm SEM, $\mathrm{n}=5)$.

Figure 3-8 Changes in body weights of ZDF rats after subcutaneous injections of PLGA (5\%) gel formulations loaded with 4\% insulin and zinc sulfate mixtures (Formulations OA-3-11, OA-3-12, OA-3-13 and OA-3-14) $($ Mean $\pm \mathrm{SEM}, \mathrm{n}=5)$ 
Figure 4-1 HPLC chromatogram of placebo of Lantus ${ }^{\circledR}$ formulation

Figure 4-2 HPLC chromatogram of Lantus ${ }^{\circledR}$ formulation ...........................................78

Figure 4-3 HPLC chromatogram of insulin glargine particles dissolved in $\mathrm{HCl}$

Figure 4-4 Blood glucose profiles in ZDF rats after subcutaneous injections of insulin (RHI), Lantus ${ }^{\circledR}$ and insulin glargine particles suspended in saline $($ dose-10 U/Kg) $($ Mean $\pm \mathrm{SEM}, \mathrm{n}=5)$

Figure 4-5 Changes in blood glucose concentrations and body weights of ZDF rats after subcutaneous injections of $4 \%$ suspension of insulin glargine particles in PBS (Mean \pm SEM, $\mathrm{n}=5$ )

Figure 4-6 Changes in blood glucose concentrations and body weights of ZDF rats after subcutaneous injections of $4 \%$ suspension of insulin glargine particles in blends of ATEC:TEC (3:1) (Mean \pm SEM, $n=5)$.

Figure 4-7 Changes in blood glucose concentrations and body weights of ZDF rats after a subcutaneous injection of gel (5\% PLGA) formulation loaded with $2 \%$ insulin glargine particles (Mean $\pm \mathrm{SEM}, \mathrm{n}=5$ )

Figure 4-8 Changes in blood glucose concentrations and body weights of ZDF rats after a subcutaneous injection of gel (5\% PLGA) formulation loaded with $4 \%$ insulin glargine particles (Mean \pm SEM, $n=5$ )

Figure 4-9 Changes in blood glucose concentrations and body weights of ZDF rats after subcutaneous injections of gel $(10 \%)$ formulations formulation loaded with $4 \%$ insulin glargine particles (Mean \pm SEM, $n=5$ )

Figure 4-10 Comparison of blood glucose concentrations in ZDF rats after subcutaneous injections of insulin glargine (4\% loading) in different formulations (Mean $\pm \mathrm{SEM}, \mathrm{n}=5$ ).

Figure 4-11 Blood glucose concentrations and concentrations of insulin glargine in serum of ZDF rats after a single subcutaneous injection of Formulation OA-G-1 (Mean \pm SEM, $\mathrm{n}=7$ ).

Figure 4-12 Blood glucose concentrations and concentrations of insulin glargine in serum of ZDF rats after a single subcutaneous injection of Formulation OA-G-4 (Mean \pm SEM, $\mathrm{n}=6)$. 
Figure 4-13 Comparison of serum glargine concentrations in ZDF rats after subcutaneous injections of 4\% insulin glargine loaded in PLGA (5\%) gel formulation (Formulation OA-G-4) and PBS

Figure 4-14 Comparison of blood glucose concentrations in ZDF rats after injections of insulin glargine (4\% loading) in different formulations with various zinc sulfate concentrations (Mean $\pm \mathrm{SEM}, \mathrm{n}=5$ ) 


\section{CHAPTER 1. INTRODUCTION}

\subsection{Diabetes mellitus}

Diabetes mellitus is a chronic disorder. It may be defined as "a metabolic disorder of multiple etiologies characterized by chronic hyperglycemia with disturbances of carbohydrate, fat and protein metabolism resulting from defects in insulin secretion, insulin action, or both" (1).

Even though diabetes has been known to humans from early historical periods, it is only recently that the WHO (World Health Organization) has recognized it as first noninfectious, worldwide epidemic (2). The cause of diabetes was unknown for centuries. Then in 1889, Joseph Von Mering and Oskar Minkowski demonstrated in dogs that the disease was associated with pancreas (3). In 1908, a German scientist, Georg Zuelzer prepared a crude extract from animal pancreas and unsuccessfully tried to treat human diabetic subjects (4). In 1921, one of the most phenomenal discoveries of medical history was done by Frederick Banting and Charles Best. They isolated an antidiabetic substance, which was later named as "isletin", from dog pancreas and injected in two pancreatectomized, hyperglycemic dogs. The blood glucose concentrations of these 'isletin' injected dogs reduced significantly. John Macleod and James Collip further purified the extract from pancreas and named 'isletin' as insulin. They successfully used insulin to treat a 14 year old diabetic boy (4). This historical treatment marked the beginning of the use of insulin in the treatment of diabetes. In 1923, Frederick Banting and John Macleod were awarded the Nobel Prize in physiology for their discovery of insulin (5). Later, Banting shared his Nobel Prize with Best, and soon after Macleod shared his Noble Prize with Collip (1).

\subsubsection{Type-1 diabetes}

It is a well established fact now that diabetes mellitus is not a single disease but a combination of different types of diseases. It is broadly categorized as diabetes type-1, diabetes type-2 and gestational diabetes (6).

The diabetes type- 1 is caused by deficiency of insulin, and has more common juvenile onset which is active in nature and requires insulin delivery for survival (6). The deficiency of insulin is caused by autoimmune destruction of beta cells in the pancreas.

The type-1 diabetes may further be divided into two classes: type-1 A and type-1 B. Type 1 A disease is due to autoimmune destruction of the beta cells in pancreas, thus reducing the biosynthesis of insulin. Antibodies against the beta cells antigen have been found in those subjects. Type-1 B disease is idiopathic, i.e. no beta cell antibodies are found in the subjects (6).

1.1.1.1 Role of genetics toward susceptibility to type-1 diabetes. It is a well recognized fact that genetics plays an important role in type-1 diabetes. For example, the 
concordance rate in monozygotic twins is much higher than the dizygotic twins (6-8). Further, some ethnic groups are more susceptible to type-1 diabetes than others. The first degree relative of type- 1 subjects are 15 fold more prone to suffer from type- 1 diabetes $(6,9)$. Davis et al. reported that the major genetic determinant of susceptibility to type-1 diabetes is HLA region of chromosome 6 (10). The insulin gene region on chromosome 11 and other confounded genes have also been implicated.

1.1.1.2 Environmental factors. The concordance rate for the development of type1 diabetes in monozygotic twins is not $100 \%$. Further, there are reports suggesting seasonal peak in the case of type-1 diabetes detection (9). This indicates of some environmental factors or the possible interaction of gene and environmental factors are playing a role in the development of type -1 diabetes (9). One of the Scandinavian studies indicated a correlation between an increase in diabetes incidences and the decline in breast feeding. The study suggested that tolerance to the common antigen might not be developed if children were exposed to cow milk protein in their early ages (11). Certain virus infection, for example Poliovirus, entero virus, and echovirus, have been suggested as the possible reasons for the autoimmune responses against the beta cells of pancreas (12). Exposer to virus infection during early childhood or pregnancy have been associated with type-1 diabetes as well (13-16).

1.1.1.3 Prevention of type-1 diabetes. There are various prevention trial studies going on around the world. The trials may be primary, secondary or tertiary. The aim of these trials is slowing the progression of type-1 diabetes (6). The primary prevention trials are before development of autoimmunity and are conducted in patients who are more prone to diabetes due to their genetic make up. Secondary preventive trials are those which are conducted in subjects showing signs of autoimmunity, but are not diabetic yet. Cyclosporine, an immunosuppressant, insulin and nicotinamide, a vitamin $\mathrm{B}_{3}$ derivative, alone or in combinations have been injected in these subjects, and development of diabetes has been studied. Some of these trials have been effective, but only for an year (17).

\subsubsection{Type-2 diabetes}

Type-2 diabetes or non insulin dependent diabetes mellitus is also known as maturity onset diabetes mellitus (18). Type-2 diabetes is a complex disorder that might be due to one or more than one gene, environmental factors or interaction of both $(18,19)$. In type- 2 diabetes, there may be no loss or some loss of beta cells, and insulin concentrations in the blood may be high, normal or low. Type-2 diabetes subjects have a high degree of genetic predisposition, and the onset is generally late $(18,20)$. Type-2 diabetes, in most cases has a slow progression. The interaction between genetic and environmental factors affects the pathogenesis of type-2 diabetes (18). Genetically predisposed subjects follow a particular trend in the progression of diabetes. The normal subjects develop insulin resistance, hyperinsuleneimia, glucose desensitization, and insulin secretion defects leading to impaired glucose tolerance followed by hyperglycemia leading to type- 2 diabetes $(18,21)$. The type- 2 diabetes may also be due to various reasons such as, malfunction of gluco-receptor on the surface of cells, so that 
they start responding to only high glucose concentration in the blood. There may be down regulation of insulin receptors leading to reduction in frequency of these receptors. The presence of hyperglycemic hormones like glucagon and physical state like obesity may also result in insulin deficiency leading to type-2 diabetes.

Glucose concentrations in the blood are controlled by three mechanisms; hepatic glucose production, peripheral tissue glucose uptake (glucose uptake by muscles adipocytes and hepatic cells) and insulin secretion by beta cells $(18,22)$. There exists a critical balance between these three mechanisms. Factors affecting any of these biochemical processes may lead to type-2 diabetes. An increase in hepatic glucose production by increasing glycogenolysis or gluconeogenesis increases glucose concentrations (23). This may be due to a decrease in the effectiveness of insulin or low insulin concentrations, or an increase in the glucagon concentrations. A combination of the above reasons may also contribute to the increased blood glucose dumping by the liver, thus leading to diabetes type-2 (24-27).

Genetic components have a strong influence in the development of type-2 diabetes (18). It has been well recognized that type-2 diabetes is multi-genetic in nature. Different candidate genes that may effect the development and progression of type- 2 diabetes have been studied. These genes have been further divided into two categories. The first category contains candidate genes for insulin secretary defects, while the second category has candidate gene for insulin resistance (18). Although various candidate genes affecting the insulin secretion and resistance have been studied, no single gene mutation that is responsible for the cause and progression of type- 2 diabetes has been identified (18).

\subsection{Insulin}

Insulin is a metabolic hormone synthesized, stored, and secreted from beta cells of pancreas. In an important landmark, Abel et al. successfully crystallized insulin in 1927 (28). Scott and Fisher in 1935 studied the role of zinc in insulin crystallization (29-31). The crystallization techniques helped in improving the yields and purity of insulin. In 1936, Hagedon came out with insulin protamine suspensions, which increased the duration of antidiabetic effect of insulin and lowered the hypoglycemic reactions (32). However, the protamine insulin was not stable and had a tendency to stick to the walls of the vials, thus reducing its potency $(33,34)$. This problem was solved by Scott and Fisher by adding zinc salts to the insulin protamine formulation and forming protamine zinc insulin. This not only stabilized the insulin protamine formulation for more than six months, but also prolonged the absorption time of insulin $(33,34)$. In 1951, HallasMoller et al. came out with an idea of Lente insulins. Semilente insulin was a suspension of amorphous insulin and zinc particles, and had a longer action than normal insulin. Ultralente was a crystalline modification (crystallized in acetate buffer) with a longer action similar to protamine zinc insulin. Lente insulin formulation contained a mixture of semilente and ultralente for rapid onset and prolonged action (35-40). In 1956, Frederick Sanger and co workers elucidated the amino acid sequence of insulin, which was found to 
be a two chain polypeptide having 51 amino acids $(41,42)$. The A-chain has 21 amino acids and the B chain has 30 amino acids. In 1958, Sanger was awarded the Nobel Prize in Chemistry for this work (43). Until then, the main source of insulin was pork or beef. Pork and beef insulin are found to be slightly different from the human insulin. Pork insulin has alanine in place of threonine at the B-30 position, whereas, the beef insulin differs in three amino acids. Thus pork insulin is more homologous to human insulin. However, due to differences in the amino acid sequences and presence of other impurities, these insulin formulations were responsible for a lot of hypersensitivity reactions $(41,42)$.

The knowledge of amino acid sequence made it possible to chemically synthesize human insulin. In 1963, Meienhofer et al. and Katsoyannis et al. successfully synthesized human insulin (44-49). Kung et al. also synthesized human insulin in 1966 (50-53). However, the amounts were never sufficient for a clinical trial and therefore, pork and bovine insulin remained in continuous use. The development in analytical and purification techniques in the 1960s led to the production of a much purer form of these animal insulins, thus reducing the cases of hypersentivity and antigenisity in the human beings. In 1967, Steiner and Oyer published the biosynthesis of insulin and described that insulin is synthesized as proinsulin in the beta cells of pancreas, and the enzymatic conversion of proinsulin in the storage vessels produces insulin $(54,55)$. Dorothy Crowfoot Hodgkin, who had been awarded a Nobel Prize in Chemistry in 1964 for the development of crystallography, later elucidated the spatial conformation of the insulin molecule (43). X-ray crystallography was further used by Adams et al. to determine the structure of insulin crystals (56).

Radioimmunoassay, for determination of very low concentrations of insulin in diabetic subjects, was developed by Rosalyn Sussman Yalow and Solomon Berson. In 1977, Rosalyn Sussman Yalow was awarded the Nobel Prize in Medicine for her work on insulin (5). In 1979, Inouye et al. completed enzymatic semi-synthesis of human insulin by using porcine insulin as a starting material. This technique immobilized trypsin, which was helpful for continuous production of insulin (57-59). Hence, industrial production of human insulin was finally feasible.

The 1970s was also an era of rapid advances in recombinant DNA technology which helped in the development of new methods for biosynthesis of human insulin. Genentech Inc. in 1978 used genetically engineered Escherichia coli to produce two chains of insulin separately and then joined the two chains chemically to produce human insulin (60). Novo Nordisk in 1982 commercially launched the first human insulin which was produced by the semi synthetic method (61). However, soon after, in the same year, Eli Lilly used Genentech's method and got approval from the US FDA to market the human insulin - the first human protein produced by the recombinant DNA technology.

\subsubsection{The structure of human insulin}

The primary structure of human insulin consists of two polypeptides (Figure 1-1). The shorter chain A, which consists of 21 amino acids (A1-A21) and the longer chain B 


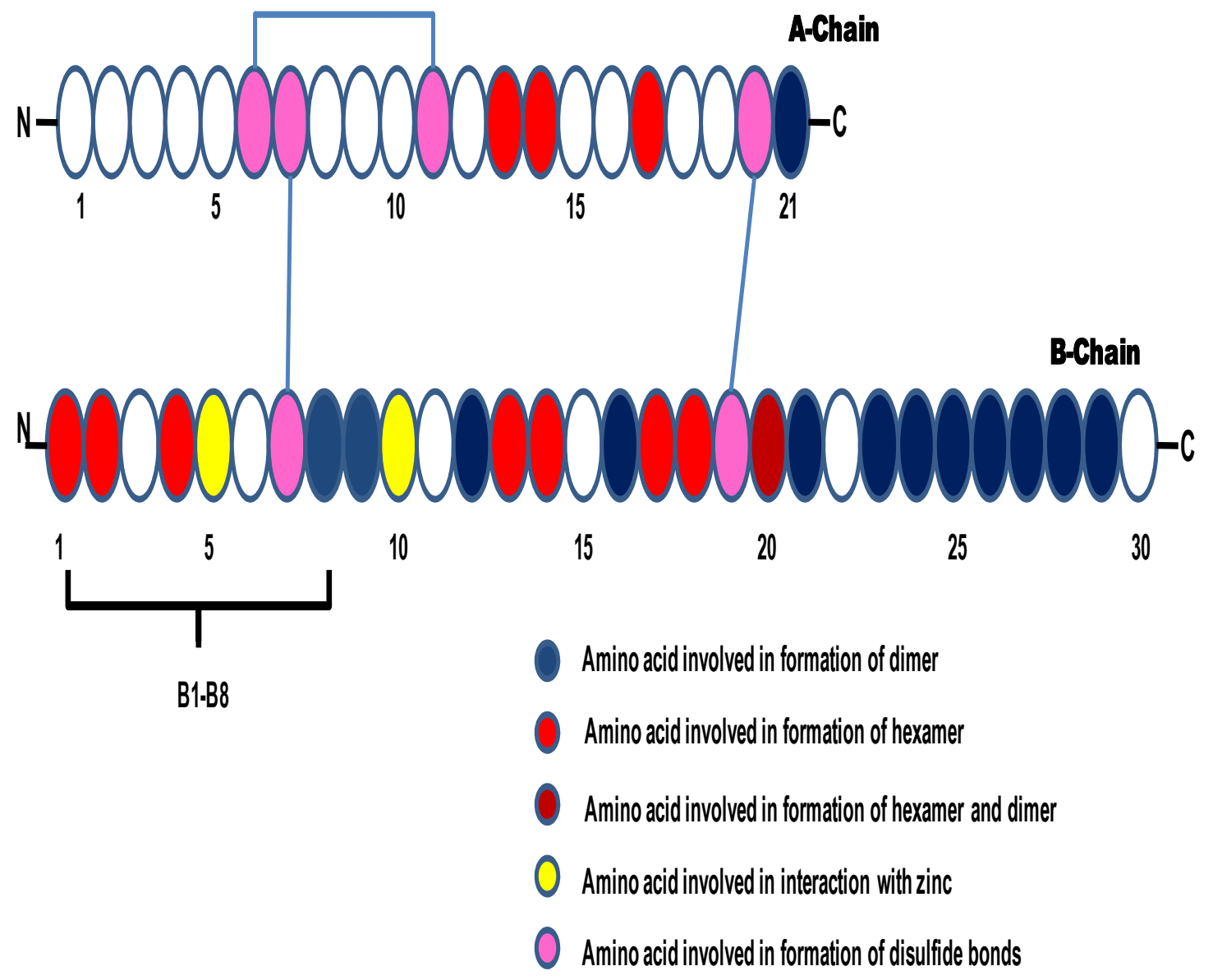

Figure 1-1 Structure of human insulin 
consists of 30 amino acids (B1-B30). The chains are connected together by two inter chain disulfide bonds between A7 and B7, and A20 and B19 cystines. In the A chain, an intra-chain disulfide bond is also present between A6 and A11 cystines. The amino acids of the two chains also interact with one another by non covalent bonds.

In three dimensional conformation of human insulin, the A chain contains two alpha helix segments-one from A2 to A8, and the another from A13 to A20. These two alpha helices are antiparallel. The N-terminal of B chain contains an alpha helix from B9 to B19, whereas, B21 to B30 forms a beta strand $(62,63)$. Wollmer et al. and Derewenda et al. explained that the A chain and the B9 to B19 structural units of B chain are stable, whereas, the conformations of $\mathrm{B} 1$ to $\mathrm{B} 8$ and $\mathrm{B} 25$ to $\mathrm{B} 30$ change in the presence of certain chemicals like phenols. In presence of phenol, the B1 to B8 segment extends to form the alpha helix (64-66).

Human insulin exists in different forms, depending on the insulin concentration, $\mathrm{pH}$ and presence of zinc ions. Monomers, i.e. single unit of insulin are only present at very low concentration $(<0.1 \mu \mathrm{M}$ or $0.6 \mathrm{mg} / \mathrm{L})(67)$.

\subsubsection{Insulin and its aggregates}

The insulin solution exits in a complex and dynamic state of equilibrium. Based on the insulin concentration, $\mathrm{pH}$ and presence of other ions, insulin may be present as monomers, dimers, tetramers or hexamers, which may further form insulin hexamer associates (67-71).

Insulin in solution at very low concentrations $(<0.1 \mu \mathrm{mol} \sim 0.6 \mu \mathrm{g} / \mathrm{ml})$ exists as monomers (single units) $(63,67,71)$. Insulin in blood is also found in predominantly monomeric form, which is also considered as the physiologically relevant form. Insulin monomer, due to its non-polar surface forces and hydrogen bond formation potentials, has a strong tendency to associate and form compact dimers (71). The dimers exist in a state of equilibrium with monomers and hexamers (71). The major interaction in monomers to form dimers occurs at B8, B9, B12, B13, B16, and C terminus segment B23 to $\mathrm{B} 28$. The two $\mathrm{COOH}$-terminal strands of $\mathrm{B}$ chain are arranged in the form of antiparallel beta sheets.

The major interaction in monomers to form dimers occurs at B8, B9, B12, B13, $\mathrm{B} 16$, and $\mathrm{C}$ terminus segment $\mathrm{B} 23$ to $\mathrm{B} 28$. The two $\mathrm{COOH}$-terminal strands of $\mathrm{B}$ chain are arranged in the form of antiparallel beta sheets. This allows close association of nonpolar forces and formation of four hydrogen bonds between B24 and B26 amino acids (71). In acidic solutions (below $\mathrm{pH} 4$ ), the insulin molecules are predominantly in dimeric state. The dimers are flexible in nature and are prone to degradation, deamidation and fibrillation in an acidic environment. This may be due to the interaction of exposed nonpolar surfaces which may interact and form heterogeneous aggregates and fibrils $(67,71)$.

The insulin hexamer, formed by the association of three dimers, is a nearly spherical structure with a height of approximately $35 \AA$ and a diameter of $50 \AA$ (71). The 
center of this spherical hexamer form is a polar channel. Two zinc ions form a coordination bond with the imidazole group of B10 histidine, which is present in the centrally located polar channel of the hexamer. The addition of zinc ions or other divalent transition metals promote the formation of hexamers $(72,73)$.

In solution, the insulin hexamers bind with metal ions and exist in three conformational states, namely, T6, T3R3 and R6. In solution, the three states of hexamers exist in a dynamic equilibrium. Addition of inorganic anions or adequate concentration of phenol like molecule shifts this dynamic equilibrium from T6 to T3R3 (72). Higher concentration of phenols further strengthens the hexamers by shifting the equilibrium completely toward the conformational state R6 (74). In all of these three conformations, the A chains folds into a helix-loop-helix motif, A2 to A8 and A12 to A19 forms two helical structures (74). The B chain can take two different conformations. The B chain in T state contains "an extended N-terminal arm", a central $\alpha$-helix (B9 to B 19), a type I turn (B20 to B23) and a C terminal B strand "In the R state, residue B1 to B9 takes up a helical conformation to form a region of alpha-helix contiguous from B1 to B9" (74).

\subsubsection{Insulin zinc and different state}

In T6 state, the six monomeric units exists in the T fold, whereas, in T3R3 state, three monomeric units exist in $\mathrm{T}$ fold and three exists in $\mathrm{R}$ fold. In the $\mathrm{R}$ state, the all six monomeric units exist in the R fold. Brange et al. reported that A13, A14, A17 residue of A chain are involved in hexamer formation (71). In B chain, B1, B2, B4, B10, B13, B14, $\mathrm{B} 17, \mathrm{~B} 18, \mathrm{~B} 19, \mathrm{~B} 20$ residues play a major role in hexamer formation. Zinc ions are key to formation of hexamers and further stabilizing them. Kadima et al. reported that T6 or two zinc "insulin hexamer is an assembly of three equivalent dimers related by 3 fold axis of symmetry" and an identical symmetry is expected in solution (75). In crystalline rhombohedral state of T6, there are two equivalent zinc binding sites. These two sites are present along the three fold axis of symmetry. In each of these two sites, there are three equivalent histidine B10 residues. Zinc forms coordinate bonds with nitrogen of imidazole side chain of these histidine. The other coordinate positions of the zinc are occupied by three water molecules or other ligands $(75,76)$. Hence, in T6 conformation state, each of the two zinc ions are coordinated with nitrogens from three His-B10 imidazole groups and three water molecules. However, Emdin et al. reported that T6 states are capable of binding more than two zinc ions. The middle of the hexamer also contains a calcium binding site. The calcium binding site is formed by the six carboxylate groups of B13 glutamate residue, and can bind three calcium ions (75).

The dimer in T6 hexamer also interacts through non-polar surfaces. The alanine B14 and leucine B17 residues of the dimer in T6 hexamer create non-polar surfaces, which further help in contact of dimers. The B1 to B8 residues are in an extended or nonhelical conformation. It has also been reported that phenylalanine B1 from one dimer interacts with leucine A13 and tyrosine A14 from the other dimer (74). Phenols and similar aromatic alcohols like cresols, and chloride ions in concentration above $6 \%$ dramatically change the structure of insulin hexamer $(67,76)$. 
Insulin crystals can be grown in the presence of chloride ions, and these crystals are similar to crystals of two zinc insulin. However, chloride ions induce a helical structure in extended residues, B1-B8 of three monomers in a hexamer, thus producing T3R3 conformation $(67,76)$. Some other lycotrophic anions like $\mathrm{SCN}^{-}, \mathrm{I}^{-}$. $\mathrm{Br}^{-}$(INS-28, 11) have also been reported to induce this $T$ to $R$ conformation in three of the six monomers, thus giving a T3R 3 conformation. This T3R3 conformation gives a new conformation to histidine at B5, which creates an additional zinc binding site (67). Two more zinc ions can coordinate with nitrogen of insulin hexamer in T3R3 conformation and hence T3R3 is also known as four-Zn insulin. However, Coffman et al. reported that in crystals, the four-Zn insulin hexamer or T3R3 state does not bind four zinc ions. They further reported an occupancy of 2.67 (77).

Monoclinic crystals are obtained on crystallization of insulin in the presence of $0.5 \%$ phenol (67). Phenols induce $\mathrm{T}$ to $\mathrm{R}$ conformation change in all six monomers of the hexamer, where the hexamer in these monoclinic crystals are in the R6 state. In helical ' $R$ ' state, all the insulin monomer subunits have the B1 to B19 helical structure (78). Since all the subunits are identical in R6 conformation, the two fold symmetry axis are restored (67). R6 conformation has two zinc ion binding sites, which lies on the symmetry axis as in T6. These two sites are again formed by His B10 imidazole, but interact with only one water molecule (78).

Phenol induced R6 hexamer also contains six identical hydrophobic pockets for binding of six phenol molecules (78). One side of the pocket is bound by B chain helix and A-chain bound the other three sides (78). Phenol molecules specifically bind to each of the six subunits at these pockets. The carbonyl oxygen of cystine A6 and amide nitrogen of A11 cystine forms hydrogen bond with hydroxyl group of phenol (67). Each of the His B5 of R6 interacts with the aromatic ring of phenols through Van der Waals forces. This blocks the axial zinc binding sites of His B5 in R6 conformation. Therefore, only two zinc binding sites are available (67).

It has been reported by several groups that $\mathrm{T}$ (extended) and $\mathrm{R}$ (helical) conformation interconvert in solution (78). Furthermore, these different conformations (T6, T3, R3, R6) exhibit different spectroscopic properties (78). Based on various studies, Kaarsholm et al. also believes that the structure of T6 and R6 are identical in solution and in the crystalline state (78).

In 1961, Schlichtkull reported that a number of metal ions such as $\mathrm{Cd}^{2+}, \mathrm{Co}^{2+}$, $\mathrm{Cu}^{2+}, \mathrm{Ni}^{2+}, \mathrm{Fe}^{2+}$, and $\mathrm{Mn}^{2+}$ can be accommodated by the zinc binding site of T6 (2-Zn insulin hexamer) (79). Roy et al. in 1990, investigated the existence of a zinc-free R6 hexamer in solution (80). Kadima et al. reported the formation of $\mathrm{Cd}^{2+}, \mathrm{Ni}^{2+}$ and $\mathrm{Fe}^{2+}$ substituted R state hexamers (75). In 1987, Wollmer et al. showed that placing nickel (II) in zinc sites impeded the transition of T to R conformation (81). In 1956, Schlichtkrull was studying the growth of very small insulin crystals, which could be injected for the treatment of diabetes, when he discovered - insulin hexamer (82-85). The monomer of insulin has 23 polar residues. All of these polar residues are present on the surface of the 
the hexamer, the two zinc ions are located on the 3-fold axis of symmetry. One is located $8 \AA$ above and the other $8 \AA$ below the 2 fold axis $(71,86)$.

\subsubsection{Insulin zinc interaction and $\mathrm{pH}$}

It is well established that insulin in the solution in neutral and alkaline $\mathrm{pH}$ range, and in the presence of zinc, predominantly exists as hexamers of molecular weight of approximately 36,000 (86). The proportion of hexamers also increases in this $\mathrm{pH}$ range as the zinc concentrate increases up to two zinc ions per hexamer (86). Hallas-Mollar et al. and Cunningham et al. reported that as the $\mathrm{pH}$ increases from $\mathrm{pH} 4.5$ to 8.0, additional seven ions of zinc can associate with a hexamer $(86,87)$. Blundell et al. reported that at neutral $\mathrm{pH}$, as the zinc concentration increases above two zinc atoms of zinc per hexamer, a 72,000 dalton unit starts appearing (86). They further reported that as the zinc amount increased above six atoms per hexamer, the distribution of insulin units became polydisperse and units with 200,000-3000,000 daltons occurred predominantly. As the $\mathrm{pH}$ was increased above $\mathrm{pH} 8.0$, even in high concentration of zinc, these polymers dissociated in 36,000 dalton hexamers in the $\mathrm{pH}$ range of 9-10 (86).

\subsubsection{Insulin mechanism of action and degradation}

Insulin binds to insulin receptors, which are located on cell membranes. The insulin receptors are present on all the cells; however, their density is highest on liver and the adipose cells. The insulin receptors are heterotetrameric glycoprotein consisting of two extracellular $\alpha$ and two transmembrane $\beta$ subunits. These subunits are linked together by disulfide bonds. The insulin binding sites are present on $\alpha$ subunits, while the have tyrosine protein kinase activity. The insulin binds to $\alpha$ subunits and induces aggregation and internalization of receptor and the bound insulin molecules. This activates a cascade of reactions resulting in the metabolic activities of insulin (88-92).

The internalized receptor insulin complex is either degraded intracellularly by insulin-degrading enzyme (IDE) or this complex is translocated back to the surface of the cell membrane, from where it may be released in systemic circulation. Insulin has plasma half life of approximately 4 to 5 minutes $(93,94)$. The liver and the kidneys are two major sites for insulin clearance from the systemic circulation (95-99). The receptor mediated clearance of insulin by hepatic and renal cells follow similar cellular mechanisms $(100,101)$. The insulin-degrading enzyme (IDE) is the major enzyme responsible for degradation of insulin. The IDE mediated degradation of insulin generates various products which have been well characterized $(93,102-108)$. It has been established that IDE recognizes the three-dimensional configuration of the insulin rather than specific peptide bonds $(93,106,108)$. Stentz et al. further reported that IDE recognizes $\alpha$-helical region around leucine-tyrosine bonds, and the major peptide bond cleavage sites of insulin are $\mathrm{A}^{13-14}, \mathrm{~A}^{14-\mathrm{I} 6}, \mathrm{~B}^{9-10}, \mathrm{~B}^{13-14}$ and $\mathrm{B}^{16-17}$ (109). These insulin fragments may not only contribute to immunoreactivity, but may also bind to insulin receptors to produce biological activity (110). 


\subsection{Treatment of diabetes}

Diabetes mellitus, depending on the type and stage of the disease, is treated with physical exercise, dietary control, oral hypoglycemics agents alone, or a combination therapy of insulin and oral hypoglycemics agents, or insulin therapy alone (111-114). Diabetics who suffer from the type-1 disease require insulin therapy for their survival. A new class of drug called amylin analogues, e.g. pramlantide acetate has also been recommended as an adjunct to insulin injections for the management of blood glucose concentrations in type-1 diabetic patients (115-117).

Depending on the stage at which the disease is diagnosed, diabetics who suffer from type- 2 disease can be treated with an appropriate diet and proper exercise, or a monotherapy or a combination therapy of oral hypoglycemic agents (114). However, most of the type- 2 diabetes patients may need insulin at some stage of their treatment for achieving the targeted glycosylated hemoglobin (HbA1c) concentrations of less than 7\% (i.e. mean plasma glucose concentration equal to $170 \mathrm{mg} / \mathrm{dL}$ or $9.5 \mathrm{mmol} / \mathrm{L})(118,119)$.

The target of insulin therapy (either alone or in combination with other antidiabetic drugs) is to mimic the physiological release and concentrations of insulin. However, the tendency of regular human insulin to self associate after a subcutaneous injection, cannot allow it to mimic physiological required insulin secretion following food intake $(113,114)$. This is because of formation of hexamer from regular human insulin at the site of injection. These hexamers cannot cross the capillary barriers due to their size. The hexamers have to dissociate first into dimers and then into monomers to cross the capillary barrier and get absorbed into the blood stream prior to their action on insulin receptors. This process of dissociation of hexamers into monomers leads to a delay in the onset of action (114).

The onset of action with a formulation of regular human insulin is reported to be from 30 min to 1 hour and the duration of action of insulin in the body is reported to be 2 to 6 hours (114). Since the duration of action of insulin is not short, it cannot mimic the rapid decline of the physiological insulin concentrations after the meals. Table 1-1 details various regular human insulin preparations. Intermediate acting and long acting insulin formulations (NPH+ Lente) have been dispensed for basal insulin requirements.

However, these formulations are associated with slow onset of action because of further stabilization of slow dissociating hexamers, and these formulations also show a pronounced peak leading to hypoglycemia in diabetic subjects. Furthermore, since the formulation like Lente and Ultralente are suspensions, the duration of action of these formulations varies significantly within and between individuals (114).

The goal of treating diabetes is to maintain a tight control on the blood glucose concentrations. It is difficult to achieve the tight glycemic control in diabetic subjects who are on regular human insulin therapy. This may lead to poor management and further progression of the disease (114). There is a strong need to develop an insulin formulation which can mimic physiological insulin action. The interest in producing the time action profile of injected insulin as per requirement of the diabetic patient is the 
Table 1-1 Insulin and modified insulin preparations

\begin{tabular}{|c|c|c|c|c|}
\hline \multirow[t]{2}{*}{ Type } & \multirow[t]{2}{*}{ Description } & \multirow[t]{2}{*}{ Examples } & \multicolumn{2}{|c|}{ Action (hours) } \\
\hline & & & Onset & Duration \\
\hline Rapid-acting & Modified insulin & $\begin{array}{c}\text { Lispro (Humalog) } \\
\text { Aspart (Novolog) } \\
\text { Glulisine (Apidra) } \\
\text { Humulin R }\end{array}$ & $>15 \mathrm{~min}$ & $3-5$ \\
\hline Short-acting & Regular insulin & Novolin R & $0.5-1$ & $5-8$ \\
\hline Intermediate-acting & $\begin{array}{l}\text { NPH or Isophane } \\
\text { Lente } \\
\text { Modified Insulins }\end{array}$ & $\begin{array}{l}\text { Humulin N } \\
\text { Novolin N } \\
\text { Humulin L } \\
\text { Glargine } \\
\text { Detemir }\end{array}$ & $\begin{array}{c}1-3 \\
1-2.5\end{array}$ & $\begin{array}{r}14+ \\
14+ \\
20-24\end{array}$ \\
\hline
\end{tabular}

Source: Chen Q. Controlled release of insulin from a novel biodegradable injectable gel system.2006, Doctoral Dissertation; Electronic Resources: http://sunzi1.lib.hku.hk. Last accessed: October 30th, 2007. (113). Used with permission. 
driving force for the development of modified insulins or insulin analogues (114).

NPH: Neutral Protamine Hagedorn insulin. The US FDA has approved five modified human insulin or insulin analogues. Table 1-1 lists these insulin analog preparations. All the formulations are to be administered via a subcutaneous injection. These insulin analogues can further be divided into two categories: Rapid acting insulin analogues, which attempt to reproduce the rapid secretion of insulin after meals, and the long acting insulin analogues (basal Insulin), which attempt to reproduce the basal concentrations of insulin that is secreted between meals during fasting and nocturnal phases $(120,121)$.

There are three rapid acting insulin analogues approved by the US FDA. Insulin Lispro (Humulog ${ }^{\circledR}$, Elli Lilly), Insulin aspart (Novolg ${ }^{\circledR}$, Novo Nordisk) and Insulin glulisine (Apidra ${ }^{\circledR}$, Sanofi-Aventis). These insulin analogues bind to the receptor of the native human insulin and produce physiological effects similar to the endogenous insulin (122). These insulin analogues have been generated and produced by using recombinant DNA technology (122). The structure of insulin in the above mentioned analogues has been modified to achieve the desired pharmacokinetic and pharmacodynamic effects (122).

Lispro was the first rapid-acting human insulin analogue that was approved by the US FDA for treating type- 1 and type-2 diabetes in humans $(113,123)$. It is a rapid acting insulin analogue which is effective for a short duration of time. The glucose lowering effect onset is from 5 to 15 minutes, and peaks in 30 to 90 minutes, and lasts for 4 to 6 hours $(32,113)$. The structure of Lispro is similar to endogenous human insulin except at two amino acid of the B chain. Lispro has been generated by inversion of amino acid lysine (B29) with proline, and proline B28 with lysine (123-125).

These modifications have affected the binding of Lispro with insulin receptor, but only slightly. However, the effects of insulin Lispro on biochemical signaling, glucose and amino acid uptake are similar to regular human insulin $(113,123)$. The minor change in the structure sequence destabilize the dimers by disrupting hydrophobic interaction and hydrogen bonding between the monomers (123). Hence Lispro monomers have a low propensity to self-associate and form dimers. It has been shown that in the presence of zinc and phenol, which are required to stabilize the Lispro formulation at neutral $\mathrm{pH}$, Lispro forms hexamers (125). However, unlike human insulin where up to six phenol molecules bind to the hexamer, only 3 phenol molecules bind to hexamers of Lispro (125). The association of monomers in Lispro hexamer has been described as relatively weaker than that of regular human insulin hexamers. Therefore, after injection, the phenols diffuse away leading to conversion of loosely associated hexamer directly into monomer $(123,125)$. The dissociation of hexamer into monomer has been described as the rate limiting step in the absorption of insulin from the site of injection (123). The elimination half-life of Lispro after a subcutaneous injection has been reported as 60 minutes. This is significantly shorter than the half-life of regular human insulin (90 minutes) after a subcutaneous injection (123). 
Lispro is recommended for type- 1 and type- 2 diabetic subjects, and should be injected within 15 minutes before meals and if necessary immediately after meals. Intravenous Lispro injection can be given in case of an emergency to deal with ketoacidosis or surgery (123). In different trials, it has been shown that Lispro is safe for fetus and new born, if it is injected in gestational diabetic subjects; however, its use is not yet recommended. Lispro can be mixed with NPH to form premixed Lispro NPH. The combination of Lispro-NPH addresses the need of both bolus and basal therapy of diabetic subjects (123).

Insulin Aspart is the second rapid acting insulin analogue approved by the US FDA. Similar to Lispro, it has faster onset and shorter duration of action $(125,126)$. Insulin Aspart is created by substitution of the proline at B28 with aspartic acid (113, $124,126)$. At the physiological $\mathrm{pH}$, a negative charge is generated on the insulin molecule. This negative charge helps in repulsion of the monomers and reduces the tendency of the monomer to self associate into hexamers. Replacement of proline with aspartic acid also leads to a loss of Van der Waals forces, necessary for self association. This leads to rapid dissociation of hexamer to monomer resulting in rapid absorption. Like Lispro, Insulin Aspart is recommended for the treatment of both type- 1 and type- 2 diabetic subjects. It is recommend to be injected immediately before meals and its duration of action lasts for approximately 2 to 4 hours (126).

Insulin glulisine (Apidra ${ }^{\circledR}$, Sanofi-Aventis) is another rapid-acting insulin analogue. In insulin glulisine, the asparagine at position B3 is replaced by lysine, and the lysine at position B29 has been replaced by glutamic acid (127). It has also a fast onset and short duration of action. One more modified insulin; is insulin glargine (Lantus ${ }^{\circledR}$ ). However, it recommended for maintaining basal insulin concentrations $(121,128)$. Insulin glargine has been altered at two different positions. In A-chain, at position 21, the asparagine has been substituted with glycine, thus imparting more resistance to deamidation in an acidic environment, and providing more stability $(128,129)$. The $\mathrm{C}$ terminus of the B-chain has elongated by the addition of two arginine molecules. This addition of two positive charges by the addition of arginines shifted the isoelectric point of the modified insulin glargine from $\mathrm{pH} 5.4$ to 6.7 (129). These modifications have made it possible to formulate insulin glargine into a slightly acidic stable solution, which is easy to inject and improve dose reproducibility. This insulin glargine solution forms stable hexamers, which precipitate at neutral $\mathrm{pH}$ at the subcutaneous site of injection, thus forming a depot of microprecipitates of the modified insulin glargine which then dissolves at a steady rate for prolonged period of time $(121,129)$.

Insulin detemir (Levemir ${ }^{\circledR}$, Novo Nordisk) is another modified recombinant human insulin whose structure has been altered such that upon subcutaneous injection, it gives a slow and an extended effect. In insulin detemir; the B-30 threonine of human insulin has been deleted $(130,131)$. A C-14 carbon fatty acid, i.e. myristic acid has been attached to B-29 lysine residue $(130,131)$. At neutral $\mathrm{pH}$, insulin detemir is soluble and does not precipitate during storage, administration or absorption (132-135). It has been reported that insulin detemir, due to its hydrophopic nature, has a tendency to bind with proteins like albumin, which are present at injection sites and in blood. This reduces its 
clearance and increases the half-life; thus leading to the extension in duration of action. Hence, insulin detemir is recommended for basal insulin therapy. It should be injected once or twice daily (132-135). Both insulin detemir and insulin glargine are recommended for the treatment of both type-1 and type-2 diabetic subjects (130).

\subsection{Non-invasive insulin delivery systems}

Subcutaneous injection of insulin has been a satisfactory mode of insulin therapy for a vast majority of diabetes subjects. However, the use of these subcutaneous injections may cause hyperinsulinemia, which may lead to hypoglycemia and diabetic micro and macro angiopathy $(136,137)$. Further, these subcutaneous injections of insulin are also associated with pain, inconvenience, physiological stress, high cost, difficulty in handling insulin, localized insulin deposition leading to local hypertrophy, and fat deposition at the site of injection $(136,138)$. Hence, during recent years, a great deal of interest has been shown for the development of novel ways to deliver insulin noninvasively.

Scientists in academic institutions as well as in the pharmaceutical industry are working on different drug delivery technologies including oral, nasal, buccal, pulmonary, transdermal, rectal and ocular to get a viable and feasible noninvasive insulin dosage form $(136,139,140)$.

\subsubsection{Oral insulin delivery of insulin}

The conventional oral route is the method of choice for delivery of most drugs because of its acceptability by patients and ease of manufacturing. Further, in the case of successful oral insulin delivery, it may mimic physiological pattern of insulin leading to better glucose homeostasis $(136,141)$. However, efficient oral insulin delivery is challenging because of enzymatic and acidic degradation, thus leading to poor and unpredictable absorption and bioavailability. Therefore, for successful oral delivery, insulin should be protected against acidic and enzymatic degradation. Moreover, its epithelial permeation should be enhanced and bioactivity should be conserved during the formulation process (136). Various groups have tried different pharmaceutical approaches to maximize insulin stability and bioavailability for effective oral insulin delivery. Some of these approaches include enzyme inhibitors, absorption enhancers, mucoadhesive polymer systems, particulate carrier delivery systems and targeted delivery systems.

Enzyme inhibitors: Insulin is extensively degraded by gastrointestinal enzymes such as trypsin, $\alpha$ chemotrypsin and elastase $(136,142)$. The effects of intestinal degradation of insulin in rats have been evaluated in the presence of different enzyme inhibitors like sodium glycocholate, camostate mesilate, bacitracin, soybean trypsin inhibitor, and aprotinin. None of these enzyme inhibitors have been reported to be effective in the small intestine, and only some of these like sodium glycocholate, camostate mesilate, and bacitracin were found to be effective in showing some 
improvement in absorption and bioavailability enhancement from the large intestine of rats. However, in general, these enzyme inhibitors are considered highly toxic and they may also affect the normal degradation and digestion of food proteins.

Absorption enhancers: Absorption enhancers improve the paracellular and transcellular transport of drugs in the gastrointestinal tract (GIT) by changing the membrane fluidity, decreasing mucus viscosity, opening tight junctions, and leakage of proteins from the membranes $(136,143)$. For improving the absorption of insulin, various non-specific permeation enhancers like bile salts, fatty acids, surfactants, salicylates, chelators, and zonula occluden toxin have been tried (144-147). However, it has been reported by Morishita et al. that the absorption enhancers increase insulin efficacy more effectively in the colon than in small intestine (147). Furthermore, since absorption enhancers increase permeation of cell membrane and open the tight junctions not only for insulin and other peptides, but also for undesirable molecules which are present in the GIT, their use may not be safe for chronic use (136).

Emisphere Technology, Inc, has developed oral capsules, called Eligen ${ }^{\mathrm{TM}}$, which use non-acylated amino acids as the carriers for transport of insulin. This oral insulin delivery system is currently undergoing Phase II clinical trials $(148,149)$.

Mucoadhesive systems for oral insulin delivery have also been developed. These systems increase the residence time of insulin at the site of absorption, strengthen the contact with the mucus, and localize the drug delivery system thus increasing the drug concentration gradient (136). Morishita et al. studied poly (methacrylic acid-g-ethylene glycol) [P(MAA-g-EG) polymers for oral insulin delivery and found that these mucoadhesive systems with smaller sized microparticular insulin loaded polymer system showed approximately $10 \%$ bioavailability of insulin after oral administration in diabetic rats (150). In a similar study, lecithin conjugated alginate microparticles, mucoadhesive chitosan nanoparticles of insulin and insulin tablets formulated with mucoadhesive thiolated (thiomers) seemed to increase the absorption of insulin via rat intestinal mucosa (151-154). These mucoadhesive delivery systems are considered to be effective in increasing the absorption of drug molecules susceptible to proteolytic degradation, however, their long term safety data in humans is warranted (155).

Colloid carrier delivery systems like microemulsions (156) liposomes (157) polymeric micelles (158) and polymeric nanoparticles and polymeric microparticles have been studied to improve the insulin delivery via oral route using specific sites like payer's patches. Other systems for oral administration of insulin that have been studied are dry insulin emulsions, solid-in-oil-in water emulsion of insulin, and microemulsion of insulin (159-162). These systems have demonstrated an improved efficacy of the orally administered insulin.

Cortecs International, Inc. in collaboration with Provalis PLC has developed oral microemulsions of insulin called Macrulin ${ }^{\mathrm{TM}}$, This system is in Phase II clinical trials (162-165). 
Different types of liposomes have also been studied for their potential as carrier for oral administration of insulin. For example, Katayama et al. reported double liposomes of insulin with aprotinin, whereas Degim et al. reported similar liposomes of insulin with sodium taurocholate and found that blood glucose concentrations decreased significantly after oral administration in rats $(166,167)$. Goto et al. prepared fusogenic liposomes of insulin with envelope glycoprotein of Sendri virus (168). These liposomes were directly administered into the lower intestine of rats and were found to improve insulin absorption. Significant decrease in blood glucose concentrations were observed in these rats $(162,168)$.

Ye et al. reported, layer-by-layer, a new method of insulin loading in alginate chitosan microcapsules. Nanocapsules of insulin made with polyethy-2-Cyano acrylate for oral delivery have also been reported. Chitosan microspheres with microadhesive properties and permeation enhancing effect have also been studied (169).

Enteric coated microspheres, using Eudragit S100 and nanoparticles using polymethacrylic acid-chitosan- polyethyle glycol have been investigated by Sajeesh et al and reported to be potential carriers for oral insulin delivery (170). Though some success has been achieved with these systems, there are still limitations such as poor drug incorporating efficacy, erratic drug release, particle aggregation, and low bioavailability, which should be addressed prior to further development (170).

Due to benefits of drug delivery to the colon, oral insulin delivery targeted in the colon has been extensively studied. Insulin delivery system with absorption enhancers like sodium glycocholate alone (171) or in combination with polyethylene oxide has shown prolonged absorption of insulin from the colon. Novel azopolymers coated pellets have been developed by Tozaki et al. for colon delivery of insulin (172).

Peyer's patches, which are present in small intestine and play a major role in absorption, are also potential targets for oral delivery of insulin. Joseph et al. prepared microspheres of PLGA for oral delivery of insulin in mice (173). The microspheres (approximately 1 micron size) were supposed to be absorbed through Peyer's patches and reported to have very significant effect on lowering of blood glucose concentrations (173).

Receptor mediated transcytosis is one more potential approach for the oral delivery of insulin. This approach does not affect the structure of plasma membranes or the paracellular junctions, and thus may be safer for chronic use. Transferrin receptors are considered good candidates for oral delivery of insulin because of their high density in the human GIT epithelium since transferrin is a natural transport protein. Xia et al. prepared insulin transferrin conjugates and reported prolonged hypoglycemia effect after oral administration in diabetic rats (174).

The buccal route of delivery for peptides and proteins like insulin is also a promising and widely investigated route of administration because of its accessibility to systemic circulation through the jugular vein. Therefore, the hepatic first pass is easily circumvented and higher bioavailability can be achieved through the use of this route. 
Moreover, using this route, insulin administration is painless, enzyme activity is low and the system can be easily removed in case of adverse effects. The peptide absorption across the buccal membrane occurs via passive diffusion (175-177). Therefore, absorption enhancer, which are non irritant and not bitter, can be used to improve the permeability and hence enhance the bioavailability of insulin. Morishita et al. reported the preparation of Pluronic F-127 gels consisting of insulin and unsaturated fatty acids for buccal delivery (177). They showed that the gels prepared with Pluronic F-127 containing unsaturated oleic acid improved the bioavailability of insulin significantly in rats $(9 \pm 7.9 \%)(177)$.

Generex Biotechnology, Inc. has developed an oral insulin system called Oral$\operatorname{lyn}^{\mathrm{TM}}$. The system is a micellar solution of insulin, which is to used as a buccal spray $(148,178,179)$. This system has been marketed in India and Ecuador.

Transferosomes, which are ultra flexible and highly deformable lipid vesicles like liposomes, consisting of cholesterol, soybean phosphotidyl choline and sodium deoxycholate have been developed by Yang et al. for buccal delivery (180). They have shown that the relative bioavailability of insulin from the buccal transferosomes was $19.78 \%$ in rabbits. Nagai et al. have shown promising results using mucoadhesive nanoparticles of insulin in beagle dogs (181). Mucoadhesive buccal tablets made of Carbopol-934 with absorption enhancers have been reported by Venugopalan et al.(182). $\mathrm{Xu}$ et al. further reported the development of buccal spray of insulin with improved hypoglycemia effect lasting for up to 5 hours (183).

\subsubsection{Nasal delivery of insulin}

Nasal cavity provides a large surface area, and delivery via nasal route avoids hepatic first pass metabolism. Therefore, nasal insulin delivery systems have been widely studied. Furthermore, it has been reported that nasal delivery of insulin resembles the physiological pulsatile pattern of insulin release (184). Various absorption enhancers like sodium deoxycholate in combination with cyclodextrins, soybean derived sterol mixtures have been used with insulin to increase its absorption via nasal membrane $(185,186)$ in rats and rabbits. This approach has shown significant absorption promoting effect. However, absorption enhancers may cause irreversible damage to the nasal mucosa upon long term use. Therefore, long term safety of these delivery systems should be studied (187). Nasal mucoadhesive systems may increase the retention and contact time in the nasal cavity, thus improving the total absorption $(188,189)$. Najafabadi et al. prepared mucoadhesive Carbopol based gel spray and reported that upon nasal administration of the gel, the relative bioavailability of insulin in rabbits was $20.6 \%$. Some other groups have also developed chitosan bioadhesive gels (190), chitosan nanoparticles (191) and chitosan microspheres (192) for nasal delivery of insulin in animals. They have all found that the absolute bioavailability of insulin has been increased significantly after nasal delivery. 
Bently Pharmaceuticals, Inc. has developed Nasulin ${ }^{\mathrm{TM}}$, a nasal spray of insulin, which utilizes absorption enhancers for increasing the bioavailability of insulin $(148,178$, 179). This system is in Phase II clinical trials.

\subsubsection{Pulmonary-lung delivery of insulin}

Pulmonary administration of proteins and peptide is considered very promising because of large surface area $\left(140 \mathrm{~m}^{2}\right)$ offered by the lungs, a thin alveolar epithelium, a lower metabolic activity of enzymes relative to GIT, and by pass of the first pass hepatic effect (136). Spray-dried insulin alone or in combination with other excipients like absorption enhancers and other particulate formulations like liposomes, nanoparticles, and nanospheres have been tried to deliver insulin successfully in animals via pulmonary route (136). Kwo et al. attempted pulmonary delivery of micro crystals of insulin and reported hypoglycemic effect lasting for seven hours in rats (193). Surendrakumar et al. prepared co-spray dried insulin powder and hyalouronic acid for inhalation and studied the insulin concentration in beagle dogs (194). They reported that the insulin-hyalouronic acid-Zn particles, after pulmonary inhalation, lasted for 4 to 5 hours. Mitra et al. studied co-administration of insulin with absorption enhancers like 1-2 dipalmitoyl phosphatidylcholine and showed a significant reduction in blood glucose concentrations after pulmonary delivery (195). Some other studies reported the use of Span 85, bacitracin, and citric acid and cyclodextrin derivatives as absorption enhancers of insulin via pulmonary route (195-197).

PLGA particles of insulin, like PLGA nanospheres, PLGA microparticles with cyclodextrins have also been prepared and reported to be effective in inducing hypoglycemia effect for 4 to 5 hours (198-200).

Exubera $^{\circledR}$, which was launched by Pfizer in January 2006 as the first non invasive insulin delivery system was a milestone in the development of noninvasive delivery of insulin. However, it was withdrawn from the market in October 2007. Though the major reason for withdrawal of the product from the market was economic loss, other issues such as occurrence of more cases of hypoglycemia $(201,202)$, dose optimization in smokers and asthmatic patients, issues such as development of antibodies against insulin, and effect of insulin on IGF-1 receptors and proliferation effects should have been more carefully studied and reported before marketing a pulmonary delivery system of insulin like Exubera ${ }^{\circledR}$ (201-205).

\subsubsection{Ocular delivery of insulin}

In 1931, Christie and Hanzal investigated the use of eye as potential delivery route for insulin for the first time (136). Ocular route provides the benefits of fast action, low immunological reactions, and by pass the first pass hepatic metabolism. However, pure insulin eye drops have low bioavailability (136). Hence, various research groups have reported the use of different absorption enhancers for ocular delivery of insulin. Yamamto et al. studied polyoxyethylene-9-lauryl ether, sodium glycocholate, sodium taurocholate, and sodium deoxycholate as absorption enhancers in eye drops of insulin, 
and studied their hypoglycemic effect and safety profiles in rabbits for three months (206). Uses of fusidic acid, dodecylmaltoside, or tetradecyl maltoside as absorption enhancers for ocular delivery of insulin have also been reported (207). One of the issues with ocular delivery of insulin is low retention time of the formulation in the ocular cavity. Positively charged liposomes containing insulin have been formulated and have been reported to reduce the blood glucose concentration in rabbits to $65-70 \%$ of initial glucose concentrations, and the effect lasted for five hours $(208,209)$. Ocular inserts like Gelfoam ${ }^{\circledR}$ have been developed for prolonged release of insulin in the eye and the system has shown lowering of blood glucose concentrations ( $60 \%$ of initial concentrations) for up to 8 hours.

\subsubsection{Rectal delivery of insulin}

The hepatic first pass metabolism can be avoided using rectum as delivery route for insulin and this route is regarded as more physiological route for administration of insulin (136). Insulin suppositories have been developed and tested in rabbits and shown to have promising results (210). Further, absorption enhancers like sodium cholate sodium glycocholate, sodium taurodeoxycholate, sodium taurocholate, sodium deoxycholate and sodium salicylate have been tried in insulin suppositories prepared with Witepsol W 35 as base. Some of these formulations showed up to 50\% relative hypoglycemic effect. Snail mucin has also been used as an absorption enhancer in glycerol gelatin suppositories of insulin by Adikwi et al.(211). They showed that blood glucose concentration reduced to $44 \%$ within two hours of application of these suppositories (211). Thermoreversible suppositories containing poloxomers, which convert to gels upon rectal application, have also been developed as rectal delivery system for insulin in animals (212).

\subsection{Controlled release of insulin}

Insulin, has been reported to be released in a pulsatile manner from isolated islets, perifused pancreas and in vivo (213). Therefore, for diabetic subjects, the ideal insulin delivery system should be capable of mimicking this natural pattern, i.e. the delivery system should have a sensing and auto shut off capability (113). Various research groups are investigating two kinds of glucose sensing insulin delivery systems. These are glucose sensitive hydrogels and artificial pancreas, i.e. closed loop delivery systems.

\subsubsection{Artificial pancreas}

These systems are also called closed loop delivery systems (113). These types of systems consist of a sensor, which remains in contact with blood or interstitial fluid, and measure the glucose concentrations in blood, and then communicate with an attached microcomputer. This microcomputer then communicates with the attached pump and modulates the release of insulin from the pump. Hence, the blood glucose concentration determines the release of insulin from the pump. However, there are limitations with 
glucose sensing and regarding the glucose turnover and insulin stability in the pumps. These kinds of systems are still in the development phase $(113,214)$.

\subsubsection{Glucose sensitive hydrogels}

These are also called "smart gels" and have the capability of changing some of their properties in response to the glucose concentration in the surroundings. $\mathrm{pH}$ sensitive polymers immobilized with the glucose oxidase have been used in many investigations $(113,215,216)$. When these systems encounter glucose, the glucose oxidase present in these systems oxidizes glucose to gluconic acid, thus lowering the $\mathrm{pH}$. The change in $\mathrm{pH}$ may lead the polymeric gel to swell and expand or collapse and squeeze. This change in dimension of polymeric gel expansion or the squeezing are proportional to the glucose concentration present in the ambience and this modulates the release of insulin from the gels made up of these polymers. Catalase has been included in these kinds of systems to enhance the oxidation rate of glucose $(113,215,216)$.

\subsubsection{Extended release of insulin}

The self regulating glucose sensitive insulin delivery systems are ideal form of insulin dosage form but they are difficult to develop and have certain limitations (113). Therefore, development of an extended release formulation of insulin, which can provide constant release of insulin for a prolonged period of time, is desirable. Long acting insulin like insulin glargine and insulin detemir, and intermediate acting insulin like NPH are available as substitutes of basal insulin. However, a daily injection of these formulations is required. This daily injection may not be tolerated by the diabetic subjects, who would need these injections for rest of their life. This may lead to poor patient compliance, which may further lead to poor management of diabetes. Hence, there is strong need to develop novel insulin formulation having prolonged action, requiring less frequent injections (113).

Non-invasive insulin delivery systems for normal delivery of insulin are still under development. Various parenteral extended release delivery systems are also being considered for the prolong delivery of insulin $(113,217)$.

Microparticles, including microspheres and microcapsules of nonbiodegradable polymer such as ethylcellulose, or biodegradable polymers such as PLGA have been prepared for prolonged delivery of insulin $(113,218-223)$. However, more focus is on the development of biodegradable microspheres. Despite being biodegradable, these biodegradable microspheres have some limitations like initial burst leading to hypoglycemia or a lag time in release $(113,221,222)$. Yamaguchi et al. reported that addition of hydrophilic excipients like glycerol or water during the preparation of these microspheres can reduce the initial burst $(113,222)$.

Further, in the microparticles prepared with PLGA, the initial burst is followed by a slow and incomplete release of insulin (218). This may be due to denaturation of insulin in the acidic environment created by the degradation products of PLGA $(218,219)$. This 
denaturation of insulin can be reduced by incorporation of some basic excipients like sodium bicarbonate $(113,218)$,). Incomplete release of insulin from such systems is a major concern and may be a potential hazard and safety issue $(218,219)$.

Implants are solid systems in form of tablets, rods, wafers, discs or films (113). These implants can be administered using a minor surgery or a trocar device. These implant systems may be biodegradable or non-biodegradable. In the case of nonbiodegradable systems, the removal of the system is necessary after depletion of the drug or after completion of the therapy. However, the biodegradable systems will degrade with time and fragment into water soluble products, which dissolve in interstitial fluid and goes away from the implant site (113).

Different kind of implantable systems have been investigated by various groups to achieve an extended release of insulin (224). Ethylene-vinyl acetate copolymers (EVAc) have been used to make discs of insulin implants. These systems could reduce the blood glucose concentrations in diabetic rats from 30 to 105 days, depending on the formulations (224-228). However, in some of these systems, only a part of the loaded insulin could be released. Furthermore, the removal of the system after completion of the release was mandatory. Implants of insulin made up of cholesterol, palmitic acid, chitosan and gellan gum have also been studied in animals (226, 228-230). PLGA and PLA biodegradable implants have also been investigated by various groups $(231,232)$.

\subsubsection{Insulin pumps}

Insulin pumps are also known as continuous subcutaneous insulin infusion (CSII). Insulin pumps were first devised by Dr. Arnold Kadish in 1960 (233-235). Relative to the initial pumps, the modern pumps are smaller in size and called as micropumps. These micropumps consist of insulin reservoir, which is a replaceable cartridge, a catheter with tubing, and a micropump. To perform, the catheter of the pump is inserted subcutaneously and connected to reservoir through the tubing. The micropump controls the release of insulin from the reservoir. The efficient use of insulin pumps requires frequent blood glucose monitoring, and accordingly, adjustment of the rate of insulin release from the pumps. Since it can cover both basal and bolus needs of insulin, better glycemic control have been reported with these kind of pumps effectively. However, stability of insulin in the reservoir, patient compliance and cost of these pumps are certain issues that are affecting the acceptability of these pumps (113).

\subsubsection{Insulin gels}

Gels are colloid systems consisting of a dispersed phase, which is dispersed in a medium resulting in a semisolid form. The gel used as drug delivery systems consists of a drug, a polymer and a solvent. The drug can be suspended or dissolved in the solvent, depending on the solubility of the drug.

Gels have been widely used for delivery of drugs via topical route. However, a few parenteral gel formulations like Eligard ${ }^{\circledR}$ and Atridox ${ }^{\circledR}$ have been marketed in the 
USA. Both of these gels use the Atirgel ${ }^{\circledR}$ technology. The Atirgel ${ }^{\circledR}$ technology combines a biodegradable polymer, PLGA with a biocompatible solvent, NMP. The drug can be dispersed in polymer solution as a suspension or dissolved as a solution. The formulation, upon subcutaneous injection, forms a depot at the site of injection, and releases the drug for a prolonged period of time. A similar formulation, Alzamer ${ }^{\mathbb{R}}$, has been developed by Alza Corporation using either ethyl benzoate or benzyl benzoate as the solvent. However, these systems suffer from initial burst, which must be controlled for successful delivery of insulin (113).

Barichello et al. reported the use of Pluronic (PF 120) gel for delivery of insulin. However, these insulin gels could only maintain low blood glucose concentrations for up to 12 hours $(113,177,236)$.

Caliceti et al. reported preparation of hydrogels which change properties with change of environment such as temperature or $\mathrm{pH}$. These gels could lower the blood glucose concentrations in diabetic rats after 2 hours after injection and maintained the effect for up to 100 hours. Kim et al.s reported the use of PLGA-PEG-PLGA polymer based gels (Relgel ${ }^{\circledR}$ ) for preparation of insulin formulations (237). The gels are liquid at room temperature, but undergo a sol gel transformation at $37^{\circ} \mathrm{C}$. Hence, an insulin containing solution of these gels when injected subcutaneously transforms to a solid and forms a depot, from which insulin can leach out for extended period of time. These formulations, upon subcutaneous injection in rats, could maintain low blood glucose concentrations for two weeks. Chen et al. made similar gels, but added zinc carbonate to them. The gel loaded with insulin Ultralente with zinc carbonate could keep the blood glucose concentrations in the normal range for 10 days in ZDF type 2 diabetic rats (113). 


\section{CHAPTER 2. PROLONGED EFFECT OF INSULIN IN PRESENCE OF WATER SOLUBLE AND WATER INSOLUBLE SOLUBLE ZINC SALTS}

\subsection{Introduction}

Insulin is a metabolic hormone synthesized, stored and secreted from beta cells of pancreas. In beta cells of pancreas insulin is stored as granules consisting of insulin hexamers. These hexamers are composed of six units of insulin loosely connected by hydrophobic forces and further stabilized by presence of zinc ions (238).

In 1937, Scott and Fisher used zinc for the first time in insulin formulations to stabilize protamine zinc insulin formulation $(31,34)$. In 1951, Hallas-Moller et al. came up with an idea of Lente insulins. Semilente was a suspension of amorphous modification of insulin and zinc, and had somewhat of a longer duration of action than the normal insulin. Ultralente was a crystalline modification of insulin (insulin crystallized in acetate buffer) with a longer duration of action similar to protamine zinc insulin formulation (35$40,82,87,239)$.

Insulin interacts with zinc ions to form hexamers, and the hexamers get further stabilized in the presence of zinc. When these hexamers are injected subcutaneously, because of their inability to cross the capillary barriers, they form a depot at the site of injection. To get absorbed into the blood stream, these hexamers have to dissociate first into dimers and then to monomers. When these hexamers are injected subcutaneously, because of their inability to cross the capillary barriers, they form a depot at the site of injection. To get absorbed into the blood stream, these hexamers have to dissociate first into dimers and then to monomers. This leads to delayed and extended effect of insulin $(68,124,238,240)$.

A novel injectable biodegradable gel system for controlled release drug delivery has been designed and characterized in our laboratory. It consists of a biodegradable polymer, PLGA, biocompatible plasticizer(s) and an active pharmaceutical ingredient (API). To date, several molecules, including antibiotics, opiate analgesics and narcotic antagonists, have been successfully incorporated into the gel system, and controlled release of the aforementioned APIs have been achieved in our laboratory.

The long term aim of this study was to develop an injectable biodegradable gel system which can be used to control the release of insulin for one week or longer, after a single subcutaneous injection in type-2 diabetic ZDF rats.

The objective of this part of the study was to investigate the effect of different water-soluble and water-insoluble zinc salts on blood glucose lowering effect of insulin in type-2 diabetic ZDF rats. 


\subsection{Materials and methods}

\subsubsection{Materials}

Insulin (RHI) powder (26.9 IU/mg containing $0.4 \%$ zinc) was purchased from Diosynth France S.A. (Usine St. Charles, France) and Humulin ${ }^{\circledR}$ U (Ultralente insulin, $10 \mathrm{~mL}$ ) (Elli Lilly and Company, Indianapolis, IN) was purchased from Cardinal Health. The HPLC analysis of insulin was performed on a Shimadzu HPLC system (Shimadzu Scientific Instruments, Inc., Columbia, MD). The primary column was a $250 \times 4.6 \mathrm{~mm}$ Alltech Macrosphere RP $300 \mathrm{C} 185 \mu \mathrm{m}$ column and the guard column (Alltech, Deerfield, IL) was $7.5 \times 4.6 \mathrm{~mm}$, packed with same material as the primary column. HPLC grade Acetonitrile (ACN), trifloroacetic acid (TFA) and distilled water were used for preparing the mobile phase. Male ZDF (fa/fa) rats (Charles River Laboratories, Inc., Wilmington, MA) weighing 300-400 grams were used for the in vivo studies. Blood glucose was measured using Bayer Ascensia ${ }^{\circledR}$ Breeze blood glucose meter (Bayer Corporation, Elkhart, IN). The $\mathrm{pH}$ of the formulations was determined using $\mathrm{pH}$ meter (Model IQ 240, IQ Scientific Instruments, Inc., San Diego CA).

\subsubsection{Methods}

2.2.2.1 Preparation of the insulin and zinc suspensions. An appropriate quantity of insulin (RHI) powder was suspended in normal saline to make $40 \mathrm{IU} / \mathrm{ml}$ suspensions. Tables 2-1-2-4 shows the composition of insulin formulations containing varying concentrations of water-soluble (zinc sulfate or zinc chloride) and water-insoluble (zinc carbonate or zinc oxide) zinc salts. The ingredients were weighed into a 7-mL glass scintillation vial and water was added to bring the final weight to approximately 3 grams. An appropriate quantity of sodium chloride was added to each formulation to make the solution isotonic before adding insulin. Humulin ${ }^{\circledR} \mathrm{U}$ suspension $(100 \mathrm{IU} / \mathrm{ml})$ was used as is.

2.2.2.2 High pressure liquid chromatography method of insulin. The HPLC analysis of RHI was performed using a Shimadzu HPLC system (Shimadzu Scientific Instruments, Inc., Columbia, MD) consisting of an SCL-10A vp system controller, a LC10 AD vp pump, a DGU-14A degasser, an SIL-10 AD vp autoinjector with a cooling system, a CTO-10A vp column oven, an SPD-10 AD vp photo-diode array (PDA) detector and a computer loaded with the Shimadzu Class-VP 7.2 software. The primary column was a $250 \times 4.6 \mathrm{~mm}$ Altech Macrosphere RP $300 \mathrm{C} 185 \mu \mathrm{m}$ column and the guard column was $7.5 \times 4.6 \mathrm{~mm}$, packed with same material as the primary column.

A gradient elution method was used for analyzing the insulin. Mobile phase A was $0.15 \% \mathrm{v} / \mathrm{v}$ trifluoroacetic acid (TFA) in water and mobile phase B was $0.13 \% \mathrm{v} / \mathrm{v}$ TFA in $95 \% \mathrm{v} / \mathrm{v}$ acetonitrile in water. Mobile phases were filtered through a Whatmann $0.45 \mu \mathrm{m}$ nylon membrane (Whatmann International Ltd, Maidstone, England) and degassed in an ultrasonicator (Model FS60, Fisher Scientific, Fair Lawn, NJ) for 10 minutes before pumping it through the HPLC system. 
Table 2-1 Composition of insulin formulations containing zinc sulfate

\begin{tabular}{ccccccc}
\hline $\begin{array}{c}\text { Formulation } \\
\#\end{array}$ & $\begin{array}{c}\text { Insulin } \\
\mathbf{( m g )}\end{array}$ & $\begin{array}{c}\mathbf{Z n S O} \\
\mathbf{( m g )}\end{array}$ & $\begin{array}{c}\text { NaCl } \\
\mathbf{( m g )}\end{array}$ & $\begin{array}{c}\text { Adjust total } \\
\text { weight with } \\
\mathbf{H} \mathbf{O}(\mathbf{g})\end{array}$ & $\begin{array}{c}\text { Amount of } \\
\text { zinc in the } \\
\text { formulation } \\
\mathbf{( m g / g )}\end{array}$ & $\begin{array}{c}\text { Molar } \\
\text { ratio of } \\
\text { insulin to } \\
\text { zinc }\end{array}$ \\
\hline OA-2-1 & 4.56 & - & 27.00 & 3.00 & - & - \\
OA-2-2 & 4.58 & 3.15 & 26.52 & 3.00 & 0.24 & $1: 14$ \\
OA-2-3 & 4.53 & 6.33 & 26.04 & 3.00 & 0.48 & $1: 28$ \\
OA-2-4 & 4.49 & 12.66 & 25.14 & 3.00 & 0.96 & $1: 56$ \\
OA-2-5 & 4.50 & 25.35 & 23.19 & 3.00 & 1.92 & $1: 112$ \\
\hline
\end{tabular}


Table 2-2 Composition of insulin formulations containing zinc chloride

\begin{tabular}{ccccccc}
\hline $\begin{array}{c}\text { Formulation } \\
\#\end{array}$ & $\begin{array}{c}\text { Insulin } \\
\mathbf{( m g )}\end{array}$ & $\begin{array}{c}\mathbf{Z n C l}_{\mathbf{2}} \\
\mathbf{( \mathbf { m g } )}\end{array}$ & $\begin{array}{c}\mathbf{N a C l} \\
\mathbf{( m g )}\end{array}$ & $\begin{array}{c}\text { Adjust total } \\
\text { weight with } \\
\mathbf{H}_{\mathbf{2}} \mathbf{O}(\mathbf{g})\end{array}$ & $\begin{array}{c}\text { Amount of } \\
\text { zinc in the } \\
\text { formulation } \\
\mathbf{( m g / g )}\end{array}$ & $\begin{array}{c}\text { Molar } \\
\text { ratio of } \\
\text { insulin } \\
\text { to zinc }\end{array}$ \\
\hline OA-2-6 & 4.61 & 1.5 & 26.07 & 3.00 & 0.24 & $1: 14$ \\
OA-2-7 & 4.51 & 3.0 & 25.14 & 3.00 & 0.48 & $1: 28$ \\
OA-2-8 & 4.62 & 6.0 & 23.28 & 3.00 & 0.96 & $1: 56$ \\
OA-2-9 & 4.59 & 12.0 & 19.56 & 3.00 & 1.92 & $1: 112$ \\
\hline
\end{tabular}


Table 2-3 Composition of insulin formulations containing zinc carbonate

\begin{tabular}{ccccccc}
\hline $\begin{array}{c}\text { Formulation } \\
\#\end{array}$ & $\begin{array}{c}\text { Insulin } \\
\mathbf{( m g )}\end{array}$ & $\begin{array}{c}\mathbf{Z n ~ C O} \mathbf{3} \\
\mathbf{( m g )}\end{array}$ & $\begin{array}{c}\mathbf{N a C l} \\
\mathbf{( m g )}\end{array}$ & $\begin{array}{c}\text { Adjust total } \\
\text { weight with } \\
\mathbf{H}_{\mathbf{2}} \mathbf{O}(\mathbf{g})\end{array}$ & $\begin{array}{c}\text { Amount of } \\
\text { zinc in the } \\
\text { formulation } \\
\mathbf{( m g / g )}\end{array}$ & $\begin{array}{c}\text { Molar } \\
\text { ratio of } \\
\text { Insulin } \\
\text { to zinc }\end{array}$ \\
\hline OA-2-10 & 4.61 & 4.82 & 27.0 & 3.00 & 0.96 & $1: 56$ \\
OA-2-11 & 4.61 & 9.71 & 27.0 & 3.00 & 1.92 & $1: 112$ \\
OA-2-12 & 4.55 & 30 & 27.0 & 3.00 & 5.98 & $1: 349$ \\
\hline
\end{tabular}


Table 2-4 Composition of insulin formulations containing zinc oxide

\begin{tabular}{|c|c|c|c|c|c|c|}
\hline $\begin{array}{c}\text { Formulation } \\
\#\end{array}$ & $\begin{array}{c}\text { Insulin } \\
\text { (mg) }\end{array}$ & $\begin{array}{l}\mathrm{ZnO} \\
(\mathrm{mg})\end{array}$ & $\begin{array}{l}\mathrm{NaCl} \\
(\mathrm{mg})\end{array}$ & $\begin{array}{c}\text { Adjust total } \\
\text { weight with } \\
\mathrm{H}_{2} \mathrm{O}(\mathrm{g})\end{array}$ & $\begin{array}{l}\text { Amount of } \\
\text { zinc in the } \\
\text { formulation } \\
\quad(\mathrm{mg} / \mathrm{g})\end{array}$ & $\begin{array}{l}\text { Molar } \\
\text { ratio of } \\
\text { insulin } \\
\text { to zinc }\end{array}$ \\
\hline OA-2-13 & 4.39 & 0.890 & 27.0 & 3.00 & 0.24 & $1: 14$ \\
\hline OA-2-14 & 4.60 & 1.79 & 27.0 & 3.00 & 0.48 & $1: 28$ \\
\hline OA-2-15 & 4.64 & 3.58 & 27.0 & 3.00 & 0.96 & $1: 56$ \\
\hline OA-2-16 & 4.44 & 7.16 & 27.0 & 3.00 & 1.92 & $1: 112$ \\
\hline
\end{tabular}


The initial mobile phase composition of $70 \%$ solvent $\mathrm{A}$ and $30 \%$ solvent $\mathrm{B}$ was maintained at a flow rate of $1 \mathrm{~mL} / \mathrm{min}$ for 2 minutes. Between 2 and 16 minutes, the percentage of solvent B was increased linearly to $50 \%$. These conditions were maintained for 3 minutes from 16 to 19 minutes. Between 19 and 24 minutes, the percentage of solvent B was decreased linearly to $30 \%$. The mobile phase was pumped at a flow rate of $1 \mathrm{~mL} / \mathrm{min}$. The injection volume of each sample, including the insulin standards, insulin controls, insulin samples (unknown concentration) and blank samples $(0.01 \mathrm{~N} \mathrm{HCl})$ without insulin was $50 \mu \mathrm{L}$. The temperature of the HPLC column was maintained at $30^{\circ} \mathrm{C}$ by using a column oven. The primary detection wavelength of insulin was set to $285 \mathrm{~nm}$.

2.2.2.3 Preparation of standard, control and sample solutions. The standard solutions and controls of insulin were prepared from recombinant human insulin. Approximately 10 or $25 \mathrm{mg}$ of insulin was weighed accurately using an analytical balance and transferred to a clean dry 10 or $25 \mathrm{~mL}$ volumetric flask. Either $1 \mathrm{~mL}$ or 2.5 $\mathrm{mL}$ of $0.01 \mathrm{~N}$ hydrochloric acid was added into the flask and the flask was swirled gently to dissolve the insulin powder. Isotonic phosphate buffered saline (PBS, $\mathrm{pH} 7.4$ ) was used to make up the volume. The resultant solution contained approximately $1 \mathrm{mg} / \mathrm{mL}$ of insulin (working stock solution). Standard solutions were prepared from working stock solution by transferring the different amounts of working stock solution to five $10 \mathrm{~mL}$ volumetric flasks and making up the volume with PBS. The final insulin standard solutions contained $5,10,25,50,75,100,125$, and $150 \mu \mathrm{g} / \mathrm{mL}$ of insulin, respectively.

The insulin controls were also prepared from the working stock solution. These controls were always prepared and dispersed into small aliquots. Each aliquot was stored in a $1 \mathrm{~mL}$ HPLC vial and sealed by Parafilm. The controls were frozen at $-20^{\circ} \mathrm{C}$ and one vial was thawed before the analysis. Depending on the approximate concentration of the insulin samples in each batch of analysis, one or two insulin controls were prepared accordingly.

2.2.2.4 Process of chromatographic.data. After each HPLC injection, the software generated the chromatograph of the sample and integrated the peak area of insulin. The peak area of the standard solutions was then plotted against the concentration of insulin.

2.2.2.5 Pharmacodynamic studies of insulin in ZDF rats. Pharmacodynamics of subcutaneously administered Humulin ${ }^{\circledR} \mathrm{U}$ (Ultralente insulin, $10 \mathrm{~mL}$ ) (Elli Lilly and Company, Indianapolis, IN) and insulin (Diosynth, Inc., Bensenville, IL) with and without varying concentrations of water-soluble and water-insoluble zinc salts was investigated in ZDF rats at a dose of $10 \mathrm{IU} / \mathrm{kg}$.

ZDF rats were randomly divided into groups of 4 to 6 animals per group. Each rat was anesthetized by isoflurane (Halocarbon Product Corporation, River Edge, NJ) using a SurgiVet/Anesco ventilator (Waukesha, WI) prior to handling the animals for injecting the insulin dosage forms and glucose measurement. Body weight of each animal was then recorded to determine the dose of the insulin suspensions to be injected. Blood glucose concentrations of each animal was measured before injecting insulin, using the 
Ascensia $^{\mathrm{TM}}$ Breeze $^{\mathrm{TM}}$ glucose monitoring system (Bayer Corporation, Elkhart, IN). Each group received one dose of a particular form of insulin formulation. An appropriate dose of insulin was injected subcutaneously on the dorsal neck of each rat using a $1 / 2 \mathrm{~mL}$ Becton Dickinson Lo-Dose ${ }^{\mathrm{TM}}$ U-100 insulin syringe with a permanently attached 28 gauge needle (0.5 inch in length) (Franklin Lakes, NJ). After injection, the rats were returned to the cages and allowed to recover from anesthesia. At pre-determined time intervals, the rats were anesthetized by the abovementioned method and blood glucose concentrations of the rats were measured. Blood glucose monitoring was stopped when the blood glucose concentrations reached their normally high concentrations.

2.2.2.6 In vitro studies. Tables 2-1-2-4 lists amounts of ingredients used to prepare various RHI formulations with and without zinc salts. After 16 hours at room temperature, the $\mathrm{pH}$ of these formulations was determined using a calibrated $\mathrm{pH}$ meter. The formulations were then centrifuged (Eppendorf Centrifuge $5415 \mathrm{C}$ ) and the supernatant were collected into HPLC vials. Insulin concentrations were determined in the supernatant using a HPLC method as specified before.

2.2.2.7 In vitro dissolution of insulin from insulin formulations with varying concentrations of zinc sulfate. The dissolution rate of RHI with and without zinc sulfate was studied using a dialysis method. Dialysis was conducted using the DispoDialyzer ${ }^{\circledR}$ ready-to-use tubes ( $500 \mu \mathrm{L}$ capacity) purchased from Spectrum Laboratories (Rancho Dominguez, CA). The_DispoDialyzer ${ }^{\circledR}$ tubes were made from cellulose ester, having a molecular weight cut-off of 50,000. The dialysis medium was isotonic PBS at pH 7.4.

Before using, the DispoDialyzer ${ }^{\circledR}$ tubes were rinsed with the dialysis media (PBS). The rinsed tubes were then kept, submerged in the dialysis media for 30 minutes prior to use.

An appropriate amount of insulin was suspended in PBS such that the concentration of insulin in the suspension was approximately $5 \mathrm{mg} / \mathrm{mL}$ (Table 2-4). In another set of samples, regular human insulin formulations with varying concentrations of zinc sulfate were prepared and used. Each formulation $(0.5 \mathrm{~mL})$ was then transferred to a DispoDialyzer ${ }^{\circledR}$ tube. Each DispoDialyzer ${ }^{\circledR}$ filled with insulin formulation was then placed into a $50 \mathrm{~mL}$ glass centrifuge tube, containing $25 \mathrm{~mL}$ of dialysis media. The DispoDialyzer ${ }^{\circledR}$ tube floated upright in the media. The centrifuge tube was capped, shaken at $100 \mathrm{rpm}$ in an orbital shaker at room temperature.

At pre-determined time intervals, the centrifuge tubes were removed from the shaker and $1 \mathrm{~mL}$ of dialysis media was withdrawn from each tube. The concentration of insulin in the media was determined by the HPLC method. The rest of the dialysis media in each centrifuge tube was dumped and $25 \mathrm{~mL}$ of fresh media was refilled. The tubes were then returned to the shaker and shaking was continued. The cumulative percentage of insulin dissolved from each suspension was plotted as a function of time. 


\subsection{Results and discussion}

\subsubsection{Pharmacodynamic studies of insulin in ZDF rats}

Figure 2-1 shows the effect insulin on blood glucose concentrations in ZDF rats after a subcutaneous injection of insulin and ultralente insulin. The blood glucose concentrations decreased from $535 \mathrm{mg} / \mathrm{dL}$ to $77 \mathrm{mg} / \mathrm{dL}$ within two hours after administering a subcutaneous injection of regular insulin. Normal blood glucose concentration ( 50 to $200 \mathrm{mg} / \mathrm{dL}$ ) was maintained in the animals for approximately 2.5 hours, and within 6 hours after injection, the blood glucose returned to its initial high concentrations of $535 \mathrm{mg} / \mathrm{dL}$. Therefore, the total duration of insulin activity in the animals was less than 6 hours. The blood glucose concentrations decreased from 431 $\mathrm{mg} / \mathrm{dL}$ to $74 \mathrm{mg} / \mathrm{dL}$ within four hours after administering a subcutaneous injection of ultralente. Normal blood glucose (50 to $200 \mathrm{mg} / \mathrm{dL}$ ) was maintained in the animals for approximately 7 hours, and within 14 hours after injection, the blood glucose concentrations returned to its initial high concentrations of $375 \mathrm{mg} / \mathrm{dL}$. Therefore, the total duration of ultralente activity was less than 14 hours.

Figure 2-2 compares the mean and standard deviations of blood glucose concentrations in ZDF rats after a subcutaneous injection of insulin with varying concentrations of zinc sulfate. It is evident from the figure that adding varying amounts of zinc from 0.24 to $1.92 \mathrm{mg}$ (obtained from zinc sulfate) to the regular insulin increased the Tmax (time at the lowest blood glucose concentration) from 2 hours (Tmax for regular insulin without any zinc) to 8 hours for formulation with $0.24 \mathrm{mg}$ of zinc and 16 hours for formulation with $1.92 \mathrm{mg}$ of zinc. The Emax (lowest blood glucose concentration) after a subcutaneous injection of RHI was $75 \mathrm{mg} / \mathrm{dL}$.

The decrease in blood glucose concentrations from approximately $500 \mathrm{mg} / \mathrm{dL}$ to approximately $110 \mathrm{mg} / \mathrm{dL}$ was observed after subcutaneous injections of RHI with 0.24 or $0.48 \mathrm{mg}$ of zinc (obtained from zinc chloride), respectively (Figure 2-3). Normal blood glucose concentrations (50 to $200 \mathrm{mg} / \mathrm{dL}$ ) were maintained for approximately 6 hours in the animals, which were injected with both the formulations, and within 10-12 hours after injecting either formulation, the blood glucose returned to its initial high concentrations of approximately $500 \mathrm{mg} / \mathrm{dL}$.

The drop in blood glucose concentrations after subcutaneous injections of insulin with 0.24 or $0.48 \mathrm{mg}$ of zinc obtained from zinc chloride or zinc sulfate was similar to the drop in blood glucose concentrations after a subcutaneous injection of ultralente in the animals. The Emax after a subcutaneous injections of ultralente (Figure 2-1) was 140 $\mathrm{mg} / \mathrm{dL}$, whereas, the Emax values after subcutaneous injections of regular insulin with 0.24 or $0.48 \mathrm{mg}$ of zinc obtained from zinc chloride or zinc sulfate was approximately $110-160 \mathrm{mg} / \mathrm{dL}$ (Figures 2-2 and 2-3). The drop in blood glucose concentrations after subcutaneous injections of insulin with 0.96 or $1.92 \mathrm{mg}$ of zinc obtained from zinc chloride or zinc sulfate was more gradual than that observed after an injection of insulin with either 0.24 or $0.48 \mathrm{mg}$ of zinc obtained from zinc chloride or zinc sulfate. Moreover, the Tmax for regular insulin with 0.96 or $1.92 \mathrm{mg}$ of zinc obtained from zinc chloride or 


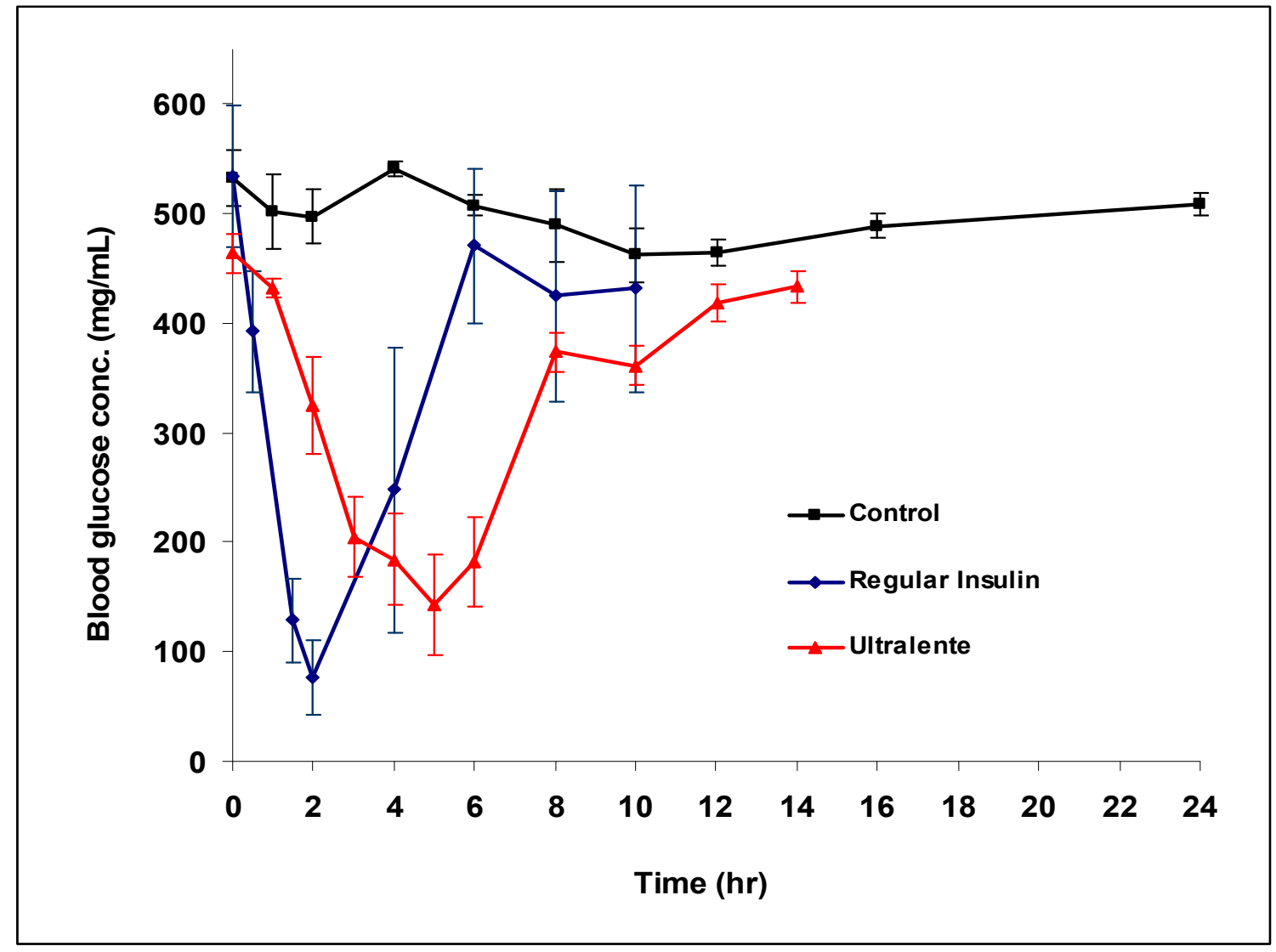

Figure 2-1 Blood glucose concentrations of ZDF rats after a single subcutaneous injection of insulin at a dose of $10 \mathrm{IU} / \mathrm{kg}($ Mean \pm SEM, $\mathrm{n}=6)$ 


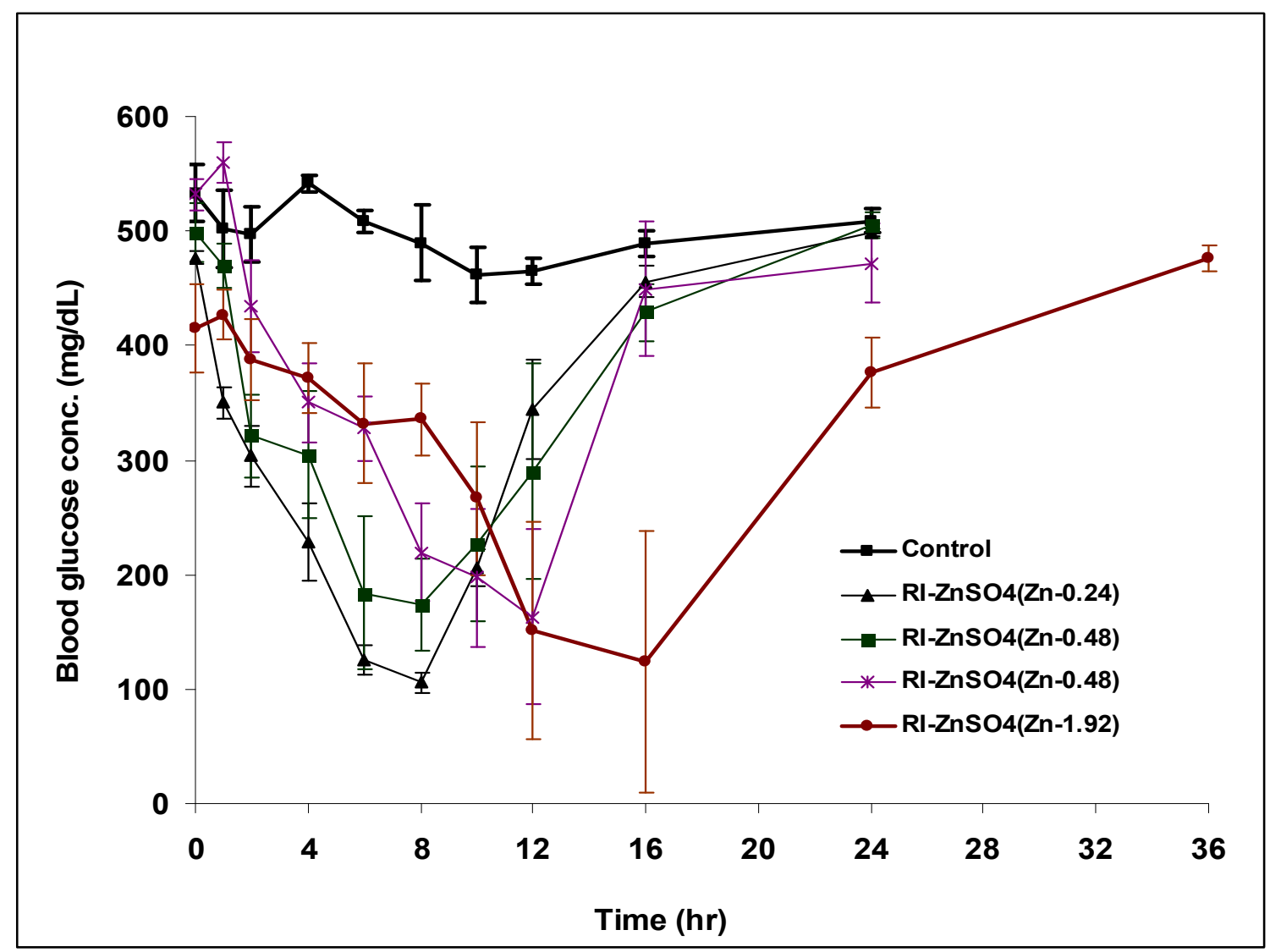

Figure 2-2 Comparison of blood glucose concentrations in ZDF rats after a single subcutaneous injection of insulin with varying concentrations of zinc sulfate (Insulin dose-10 U/kg) (Mean \pm SEM, $n=4)$ 


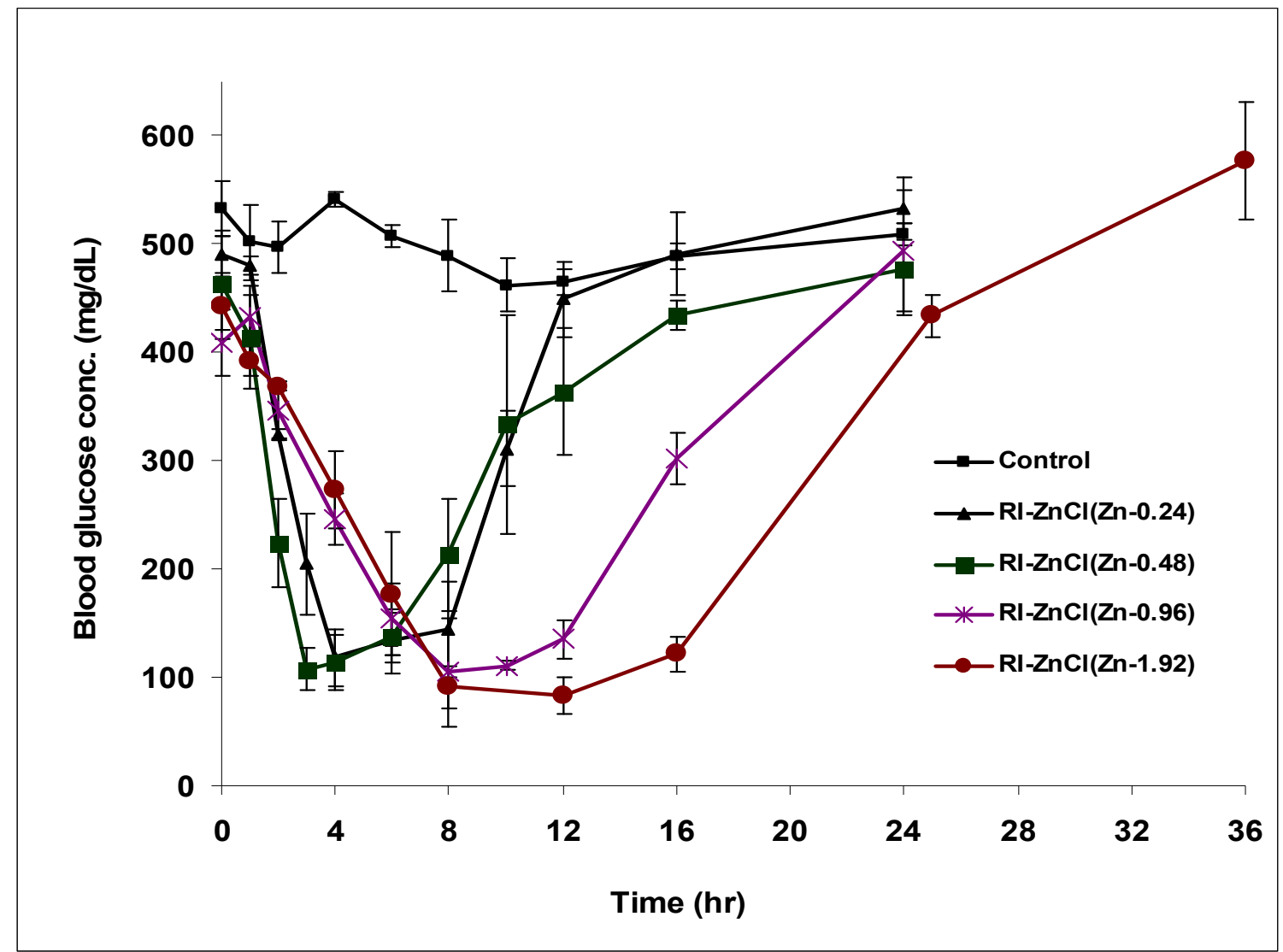

Figure 2-3 Comparison of blood glucose concentrations in ZDF rats after a single subcutaneous injection of insulin with varying concentrations of zinc chloride (Insulin dose-10 U/kg) (Mean \pm SEM, $n=4)$ 
zinc sulfate was 12 or 16 hours, respectively (Figures 2-2 and 2-3). Hence, insulin release could be extended by varying concentrations of both the water soluble salts of zinc.

Moreover, the effect of different concentrations of zinc obtained from two different water soluble salts on the Tmax and Emax were similar. However zinc chloride, due to its very hygroscopic nature, was not used further in these studies.

Figure 2-4 and Figure 2-5 compare the mean and standard deviations of blood glucose concentrations in ZDF rats after a subcutaneous injection of insulin with varying concentrations of water-insoluble zinc salts. Figure 2-4 depicts the blood glucose concentrations after subcutaneous injection of insulin with different concentrations of zinc, obtained from zinc carbonate. It is evident from the Figure 2-4 that adding varying amounts of zinc from 0.96 to $1.92 \mathrm{mg}$ (obtained from zinc carbonate) to the insulin increased the Tmax (time at the lowest blood glucose concentration) from 2 hours (Tmax for regular insulin without any zinc) to only approximately 4 hours for formulation with $0.96 \mathrm{mg}$ and $1.92 \mathrm{mg}$ of zinc. However, the blood glucose concentrations reached back to high concentrations of $500 \mathrm{mg} / \mathrm{dL}$ within 8 hours.

Figure 2-4 and Figure 2-5 compare the mean and standard deviations of blood glucose concentrations in ZDF rats after a subcutaneous injection of insulin with varying concentrations of water-insoluble zinc salts. Figure 2-4 depicts the blood glucose concentrations after subcutaneous injection of insulin with different concentrations of zinc, obtained from zinc carbonate. It is evident from the Figure 2-4 that adding varying amounts of zinc from 0.96 to $1.92 \mathrm{mg}$ (obtained from zinc carbonate) to the insulin increased the Tmax (time at the lowest blood glucose concentration) from 2 hours (Tmax for regular insulin without any zinc) to only approximately 4 hours for formulation with $0.96 \mathrm{mg}$ and $1.92 \mathrm{mg}$ of zinc. However, the blood glucose concentrations reached back to high concentrations of $500 \mathrm{mg} / \mathrm{dL}$ within 8 hours.

Figure 2-4 and Figure 2-5 compare the mean and standard deviations of blood glucose concentrations in ZDF rats after a subcutaneous injection of insulin with varying concentrations of water-insoluble zinc salts. Figure 2-4 depicts the blood glucose concentrations after subcutaneous injection of insulin with different concentrations of zinc, obtained from zinc carbonate. It is evident from the Figure 2-4 that adding varying amounts of zinc from 0.96 to $1.92 \mathrm{mg}$ (obtained from zinc carbonate) to the insulin increased the Tmax (time at the lowest blood glucose concentration) from 2 hours(Tmax for regular insulin without any zinc) to only approximately 4 hours for formulation with $0.96 \mathrm{mg}$ and $1.92 \mathrm{mg}$ of zinc. However, the blood glucose concentrations reached back to high concentrations of $500 \mathrm{mg} / \mathrm{dL}$ within 8 hours.

Therefore, there was not much difference in the blood glucose concentrations of ZDF rats after subcutaneous injections of formulations of insulin containing zinc carbonate at two different zinc concentrations of 0.96 to $1.92 \mathrm{mg}$ (Figure 2-1). Furthermore, the total duration of action in the animals for these formulations was shorter than that of Ultralente. Figure 2-5 depicts the blood glucose concentrations after a subcutaneous injection of insulin with different concentrations of zinc obtained from zinc oxide (another water-insoluble zinc compound). It is clear from the Figure 2-5 that 


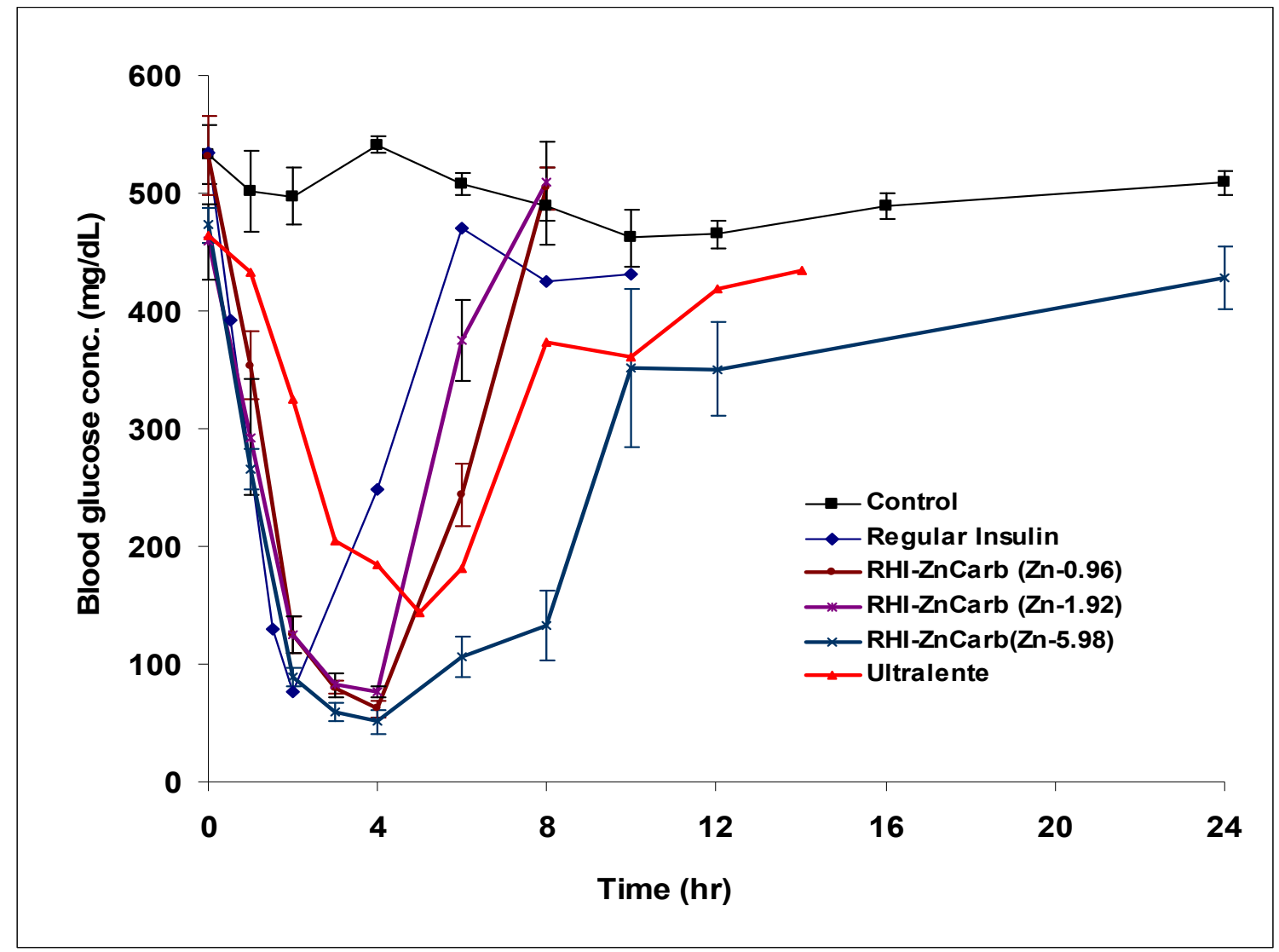

Figure 2-4 Comparison of blood glucose concentrations in ZDF rats after subcutaneous injection of insulin, ultralente and insulin with varying concentrations of zinc carbonate (Insulin dose-10 U/kg) (Mean \pm SEM, $\mathrm{n}=4)$ 


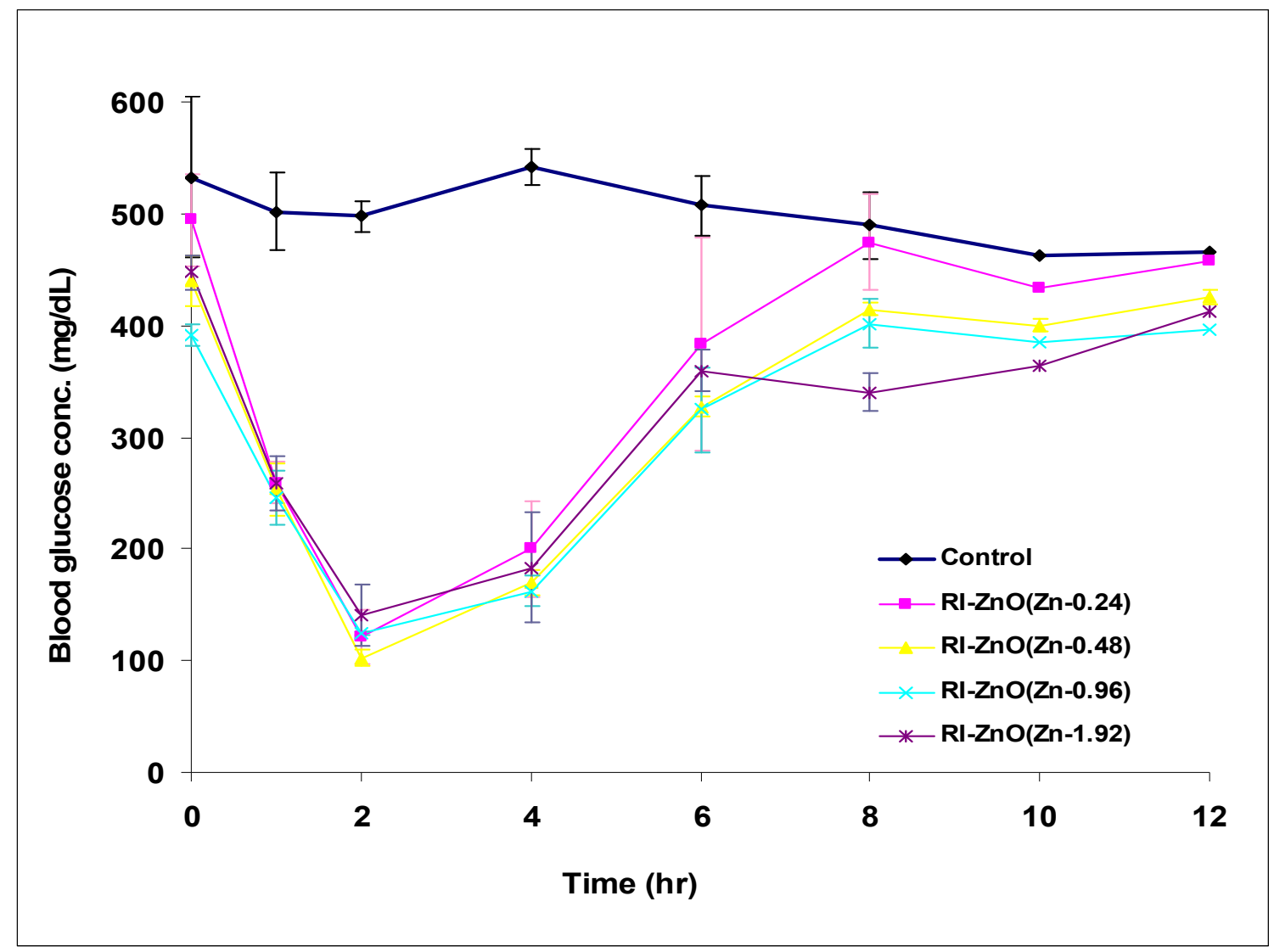

Figure 2-5 Comparison of blood glucose concentrations in ZDF rats after a single subcutaneous injection of insulin with varying concentrations of zinc oxide (Insulin dose$10 \mathrm{U} / \mathrm{kg})($ Mean \pm SEM, $\mathrm{n}=4)$ 
increasing the amount of zinc oxide in the suspension formulation of insulin does not prolong the blood glucose lowering effect of insulin.

Figure 2-6 shows a comparison of blood glucose concentrations in ZDF rats after subcutaneous injections of RHI with water-insoluble zinc carbonate and zinc oxide, and water-soluble zinc sulfate and zinc chloride. (Insulin dose-10 U/kg). It is evident from Figure 2-6 that the blood glucose lowering effect of insulin and maintenance of low blood glucose concentrations for a prolonged period of time in the ZDF rats was more than for the formulations prepared with water-soluble zinc salts than those prepared with waterinsoluble salts. This could be due to complexation of zinc ions (obtained from the watersoluble salts) with the insulin and formation of an insoluble zinc-insulin hexamers. The hexamers could have been further stabilized to form higher molecular weight associates in presence of excess free zinc ions (86).

These high molecular weight insulin associates (72000-256,000 Dalton) and hexamers (36000 Dalton) cannot cross the capillary barrier, and can only reach the blood stream after dissociation to monomers (86). This explains the reason of slow and prolonged effect of insulin in presence of high concentration of water soluble-salts like zinc chloride and zinc sulfate. The water-insoluble zinc salts, on the other hand, could not dissolve in the aqueous medium and hence could not provide the zinc ions to complex with the insulin molecules.

\subsubsection{In vitro release of insulin from insulin formulations with varying concentrations of zinc sulfate}

To further investigate the effect of zinc on RHI formulations, in vitro release studies were performed on insulin formulations prepared with water soluble zinc sulfate. Figures 2-7 and 2-8 depict the percentage release of insulin from suspensions of insulin prepared without and with varying concentrations of zinc sulfate, respectively, across a semi permeable membrane (dialysis bags with a MWCO of 50,000). Figure 2-7 shows that approximately 100 percent of insulin was released within 12 hours through the semipermeable membrane from the RHI formulation without any additional zinc sulfate. However, as shown in Figure 2-8, the release of insulin from RHI formulations prepared with two different concentrations of zinc $(0.24$ and $0.48 \mathrm{mg} /$ gram of formulation) obtained from zinc sulfate was only approximately three percent at 48 hour time point. Moreover, no insulin was released from these formulations until 4 hours, and no detectable quantity of insulin was observed from RHI formulations prepared with 0.96 and $1.92 \mathrm{mg} / \mathrm{g}$ of zinc (obtained from zinc sulfate) until 48 hours. The contents of dialysis bags containing RHI formulations were studied after 48 hours of release study.

Figure 2-9 depicts the percentage of insulin remaining in the dialysis bags after completion of the in vitro release study (i.e. 48 hours), with RHI formulations prepared with varying concentration of zinc. The results of the study also showed that after 48 hours of in vitro release study, approximately 3 percent of insulin was remaining inside the dialysis bags containing insulin formulations without any zinc.However, in the case 


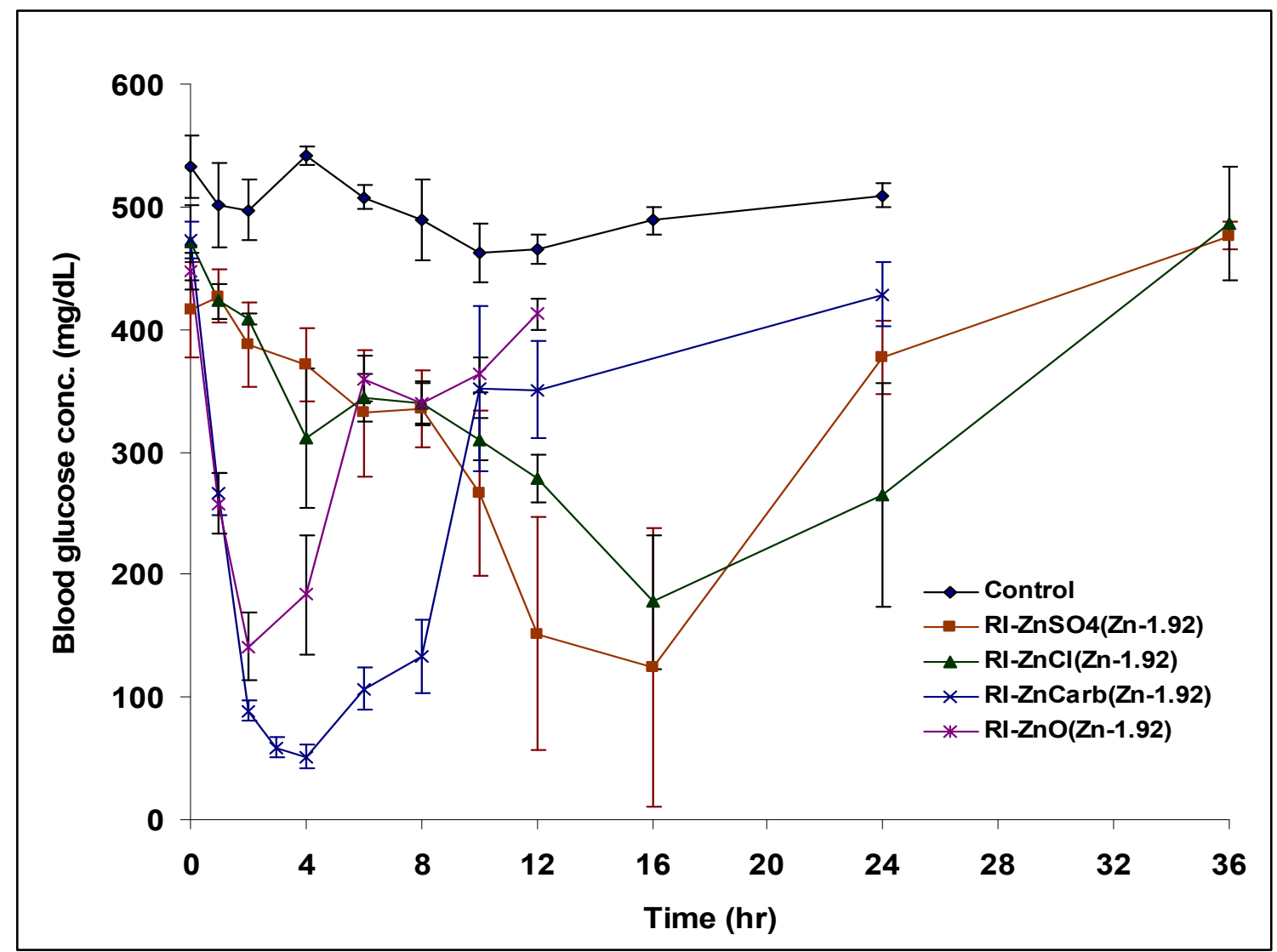

Figure 2-6 Comparison of blood glucose concentrations in ZDF rats after a single subcutaneous injection of insulin with water-soluble and water-insoluble zinc salts (Insulin dose-10 U/kg) (Mean \pm SEM, $\mathrm{n}=4)$ 


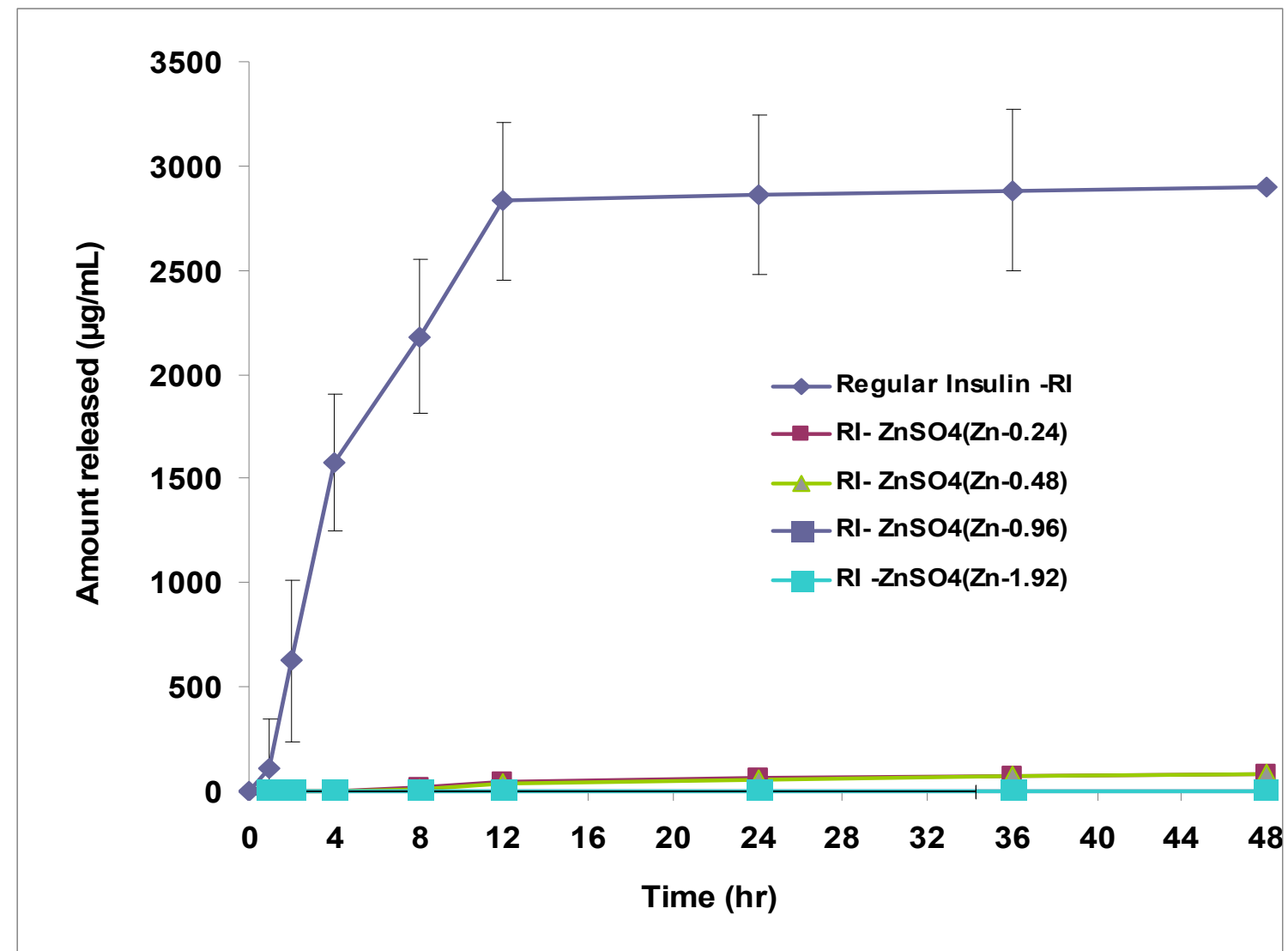

Figure 2-7 Comparision of in vitro insulin release from insulin suspension formulations with and without zinc sulfate 


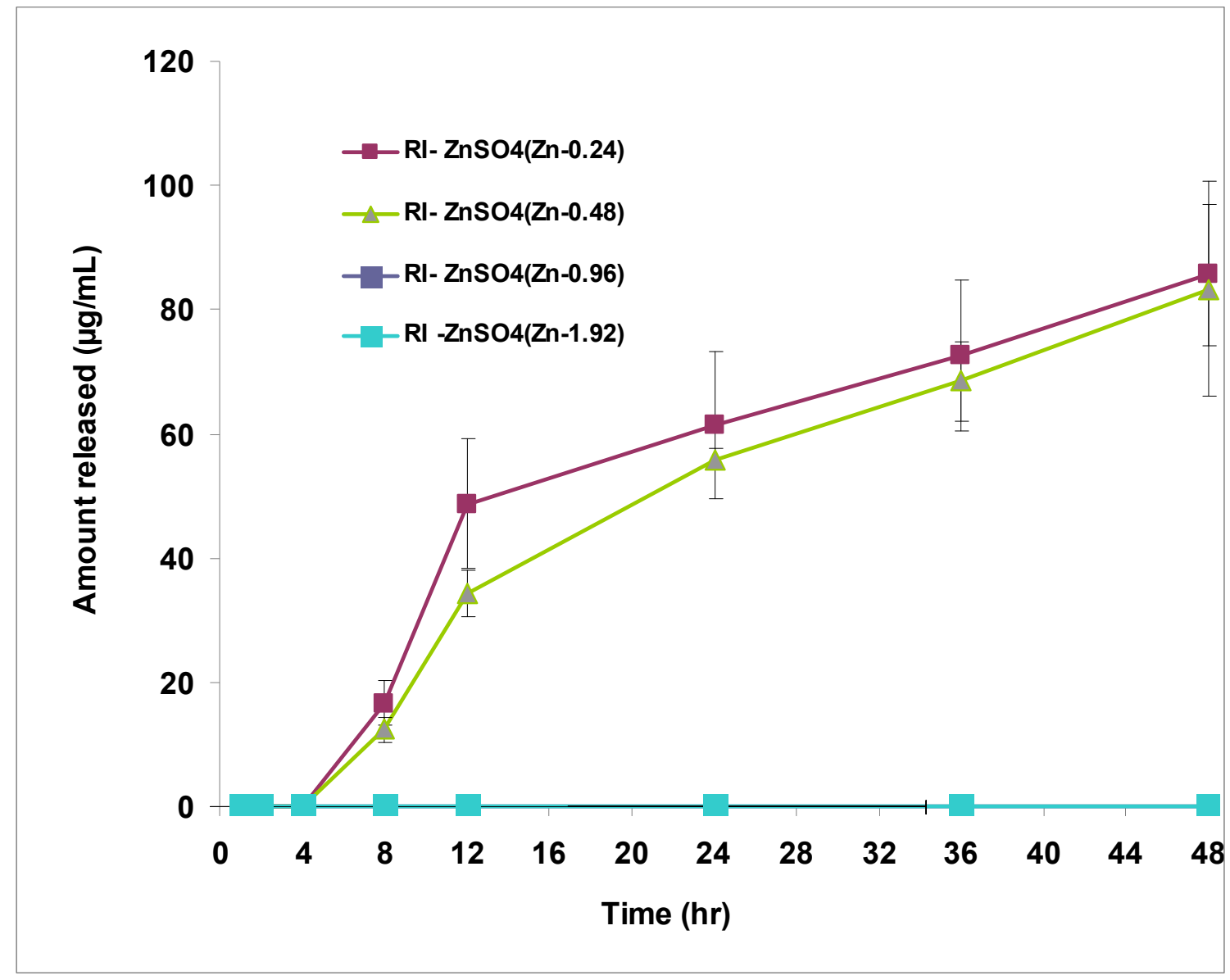

Figure 2-8 Comparision of in vitro insulin release from insulin suspension formulations with zinc sulfate 


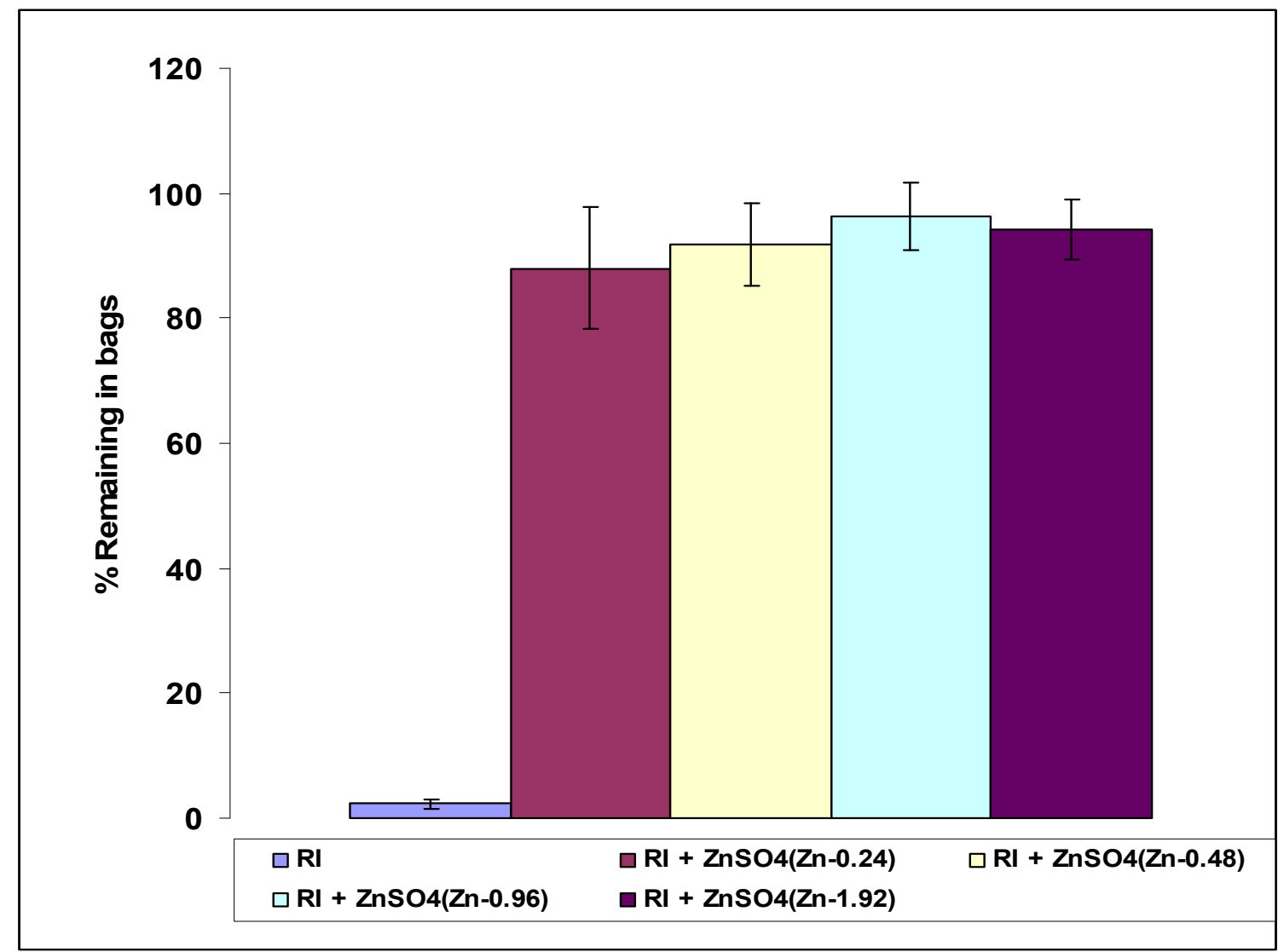

Figure 2-9 Percentage of insulin remaining in the dialysis bags after completion of in vitro release study of insulin formulations with varying concentrations of zinc 
of insulin formulations prepared with varying concentrations of zinc sulfate, settled insulin powder could be seen at the bottom of the dialysis bags.Quantitation of insulin concentration in these dialysis bags showed that approximately 88 to 96 percentage of insulin was still present inside the dialysis bags even after 48 hours.

This further confirmed that fact that insulin forms hexamers and other complex associates of high molecular weight, which could not cross the dialysis bag with a MWCO of 50,000. To see the effect of $\mathrm{pH}$ on the concentration of insulin in the supernatant, insulin formulations with $0.24 \mathrm{mg}$ of zinc/g of formulation (similar to Formulation OA-2-2) and without zinc (similar to Formulation OA-2-1) were prepared at different $\mathrm{pH}$ values of approximately 2.0, 3.0, 4.0, 5.0, and 5.6. Figure 2-10 shows the concentration of RHI in the supernatant obtained from these formulations at different $\mathrm{pH}$ values. It is clear from the figure that, as the $\mathrm{pH}$ decreased from 5.6 to 4.0 , the amount of insulin dissolved in the supernatant increased from approximately $50 \mu \mathrm{g} / \mathrm{mL}$ to 1000 $\mu \mathrm{g} / \mathrm{mL}$. As the $\mathrm{pH}$ decreased further to $\mathrm{pH} 2.0$, the amount of insulin dissolved in the supernatant increased up to $1500 \mu \mathrm{g} / \mathrm{mL}$, i.e. insulin dissolved completely at $\mathrm{pH} 2.0$. This is in agreement with the prior findings that insulin dissolves at lower $\mathrm{pH}$ values $(238$, 241).

Figure 2-10 also shows the concentration of insulin in the supernatant in formulations prepared with $0.24 \mathrm{mg}$ of zinc/ gram of formulation (similar to Formulation OA-2-2) at different $\mathrm{pH}$ values of approximately 2.0, 3.0, 4.0, 5.0, and 5.6. It is evident from the figure that the concentration of insulin in the supernatant in the presence of zinc was approximately $40 \mu \mathrm{g} / \mathrm{mL}$ and $100 \mu \mathrm{g} / \mathrm{mL}$ at $\mathrm{pH} 5.0$ and 4.0 , respectively. These insulin concentrations are considerably less than the insulin concentrations (approximately $400 \mu \mathrm{g} / \mathrm{mL}$ and $100 \mu \mathrm{g} / \mathrm{mL}$ ) in the supernatant of the insulin formulations without zinc, at the similar $\mathrm{pH}$ values of 5.0 and 4.0. This may be because of interaction of insulin with zinc, leading to the formation and precipitation of insulin hexamers out of the solution. However at pH 3.0 and 2.0, the insulin concentrations in the supernatant of both the formulations prepared with and without zinc were similar. This also proves that insulin does not interacts with zinc at lower $\mathrm{pH}$ values (86).

Figure 2-11 shows the $\mathrm{pH}$ and concentration of insulin in the supernatant obtained from formulations of insulin with varying concentrations zinc sulfate. The $\mathrm{pH}$ of insulin formulation without any additional zinc sulfate was approximately 5.6. As zinc sulfate was added to this insulin formulation, the $\mathrm{pH}$ decreased from 5.6 to approximately 5.05. With further addition of zinc sulfate, the $\mathrm{pH}$ decreased up to 4.85. The isoelectric point of insulin is 5.4 (238). Since insulin is more soluble in the acidic medium, as the $\mathrm{pH}$ went down towards the more acidic $\mathrm{pH}$, i.e. away from the isoelectric point of insulin, more insulin dissolved in the medium. However, when these insulin formulations with varying concentrations of zinc sulfate were centrifuged and concentrations of insulin were determined in the supernatant, an opposite trend was observed.

Figure 2-11 also shows the concentration of insulin in the supernatant obtained from formulations of insulin prepared with varying concentrations zinc sulfate. As shown in Figure 2-11, as the zinc sulfate concentration increased in the formulations, the $\mathrm{pH}$ of 


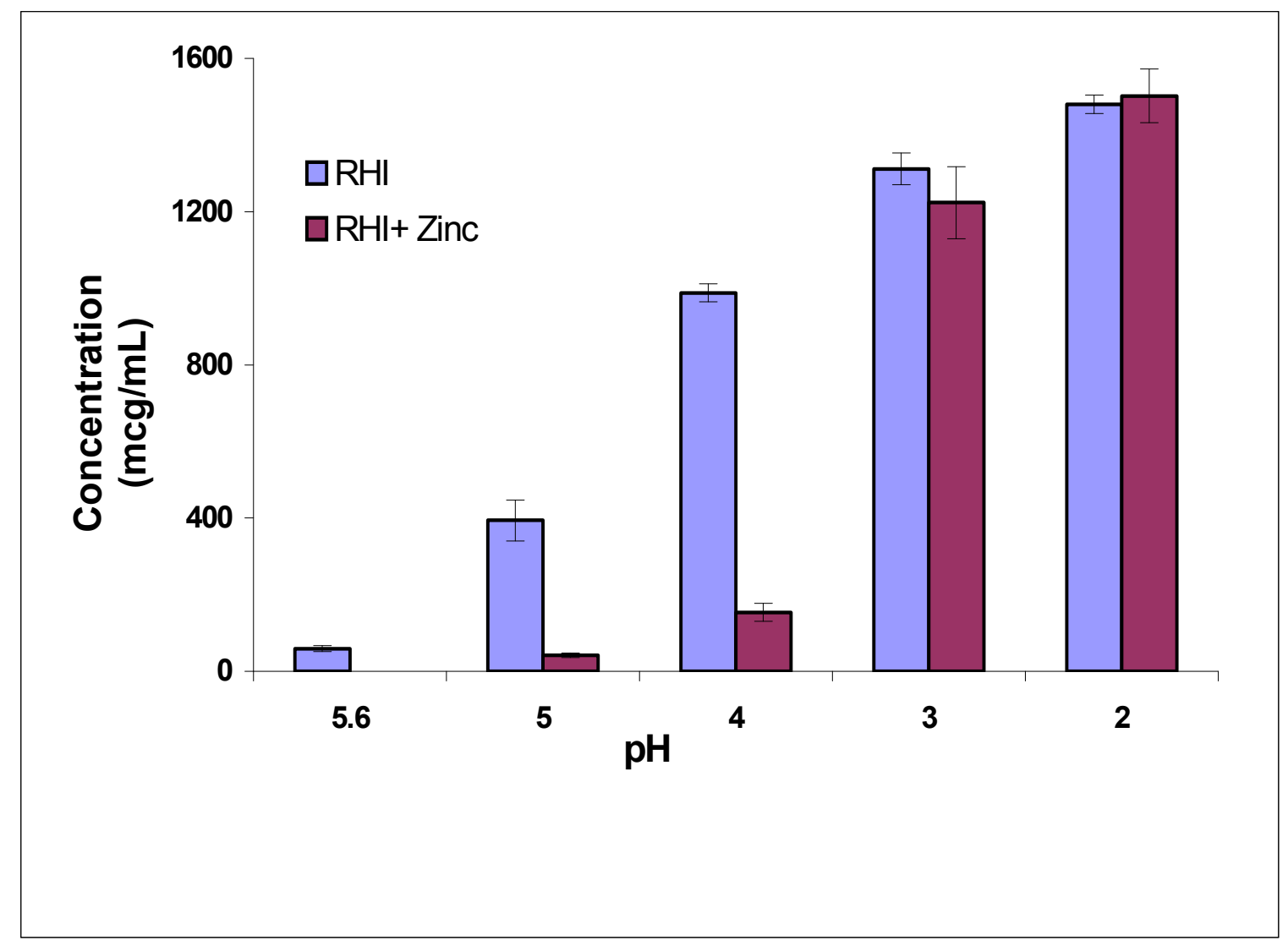

Figure 2-10 Concentrations of insulin in the supernatant obtained from formulations of insulin alone or insulin with zinc sulfate $(0.24 \mathrm{mg} / \mathrm{mL})$ at different $\mathrm{pH}$ values 


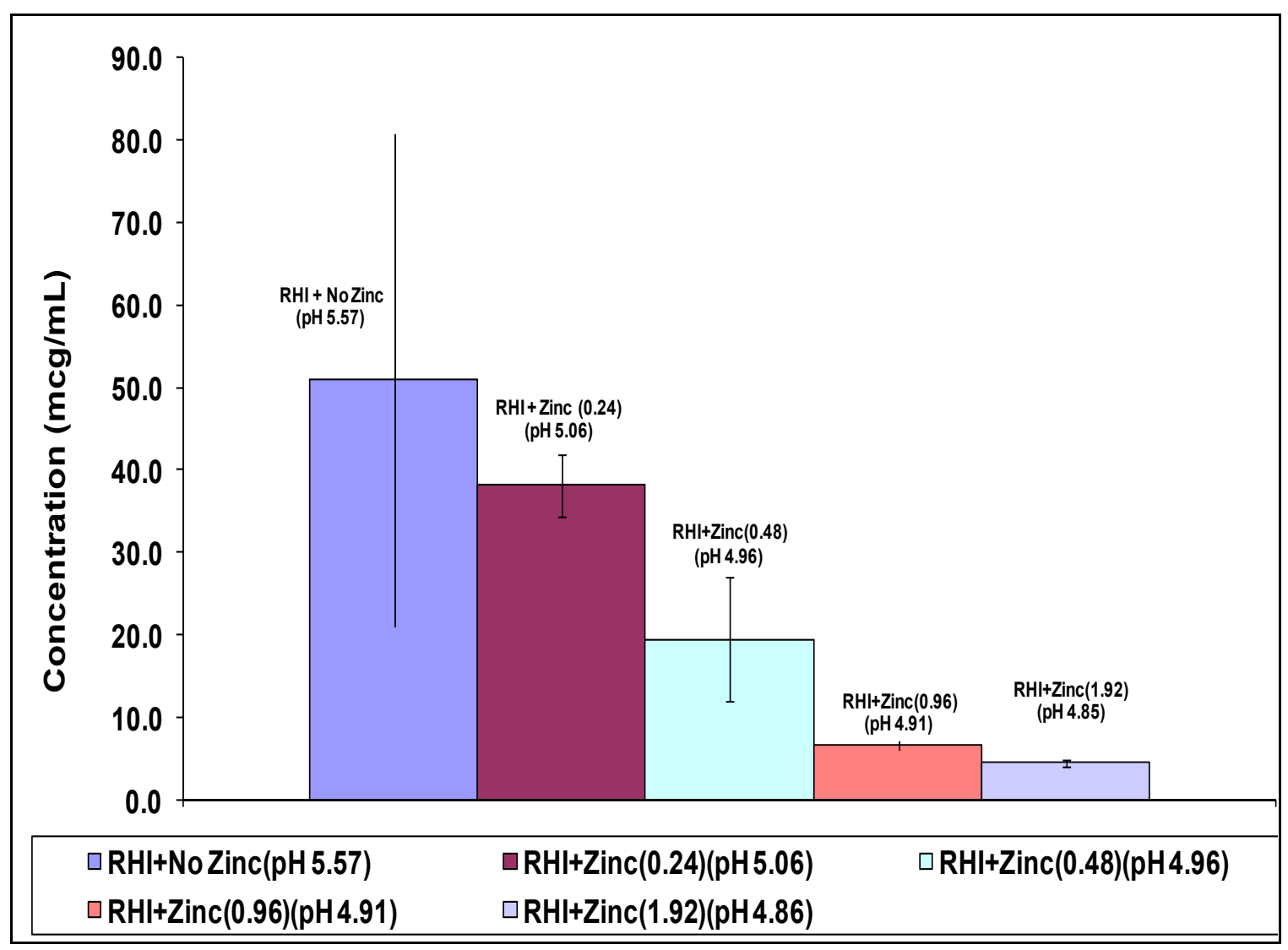

Figure 2-11 $\mathrm{pH}$ and concentration of insulin in the supernatant obtained from formulations of insulin (RHI) with varying concentrations zinc sulfate 
the formulation decreased from 5.57 to 4.85 . However, as the zinc sulfate concentration increased in the formulations, the insulin concentration in the supernatant of the formulation also decreased from approximately $50 \mu \mathrm{g} / \mathrm{mL}$ to $5 \mu \mathrm{g} / \mathrm{mL}$. This approximately 10 fold decrease, in insulin concentration in the supernatant of the formulations may be due to an increase in the zinc ions in the formulations.

To investigate this phenomenon further, four different formulations were prepared at the final $\mathrm{pH}$ of approximately 5.05, 4.95, 4.90 and 4.85 . These final $\mathrm{pH}$ values were selected based on the $\mathrm{pH}$ values of formulations of insulin with varying concentrations zinc sulfate. The insulin concentration was determined in the supernatant of these formulations after similar treatment as of formulations of insulin with varying concentrations zinc sulfate. As indicated in Figure 2-12 the insulin concentration, in the supernatant of the insulin formulations was much lower in the presence of zinc at similar $\mathrm{pH}$ values.

Figure 2-13 depicts the possible explanation of this finding. In the formulations of RHI with varying concentrations zinc sulfate, insulin was in suspension and zinc ions were in solution. With the addition of more zinc sulfate, the $\mathrm{pH}$ of the medium decreased and the medium became acidic. Since the solubility of insulin is more at the acidic $\mathrm{pH}$, more insulin solublized at lower $\mathrm{pH}$ and hence was available to form hexamers in the presence of excess of zinc ions. As more hexamers and loose associates of hexamers were formed, they precipitated out of the solution thus resulting in a decrease in the net insulin concentration in the supernatant. When these insulin formulations with varying concentrations of zinc and containing hexamers were administered to the ZDF rats, the insulin hexamers and loose associates could not cross the capillary barrier; and thus formed a depot at the site of the injection. This depot formation may be the reason for the prolonged in vivo effect of the insulin formulations prepared with varying concentrations zinc sulfate, after subcutaneous injections in the ZDF type 2 diabetic rats.

\subsection{Conclusions}

The blood glucose lowering effect of insulin could be extended by varying concentrations of both the water-soluble salts of zinc. However, the blood glucose lowering effect of insulin and maintenance of low blood glucose concentrations for a prolonged period of time in the ZDF rats was more than for the formulations prepared with water-soluble zinc salts than those prepared with water-insoluble salts. This could be due to complexation of zinc ions (obtained from the water-soluble salts) with the insulin and formation of an insoluble zinc-insulin hexamers. 


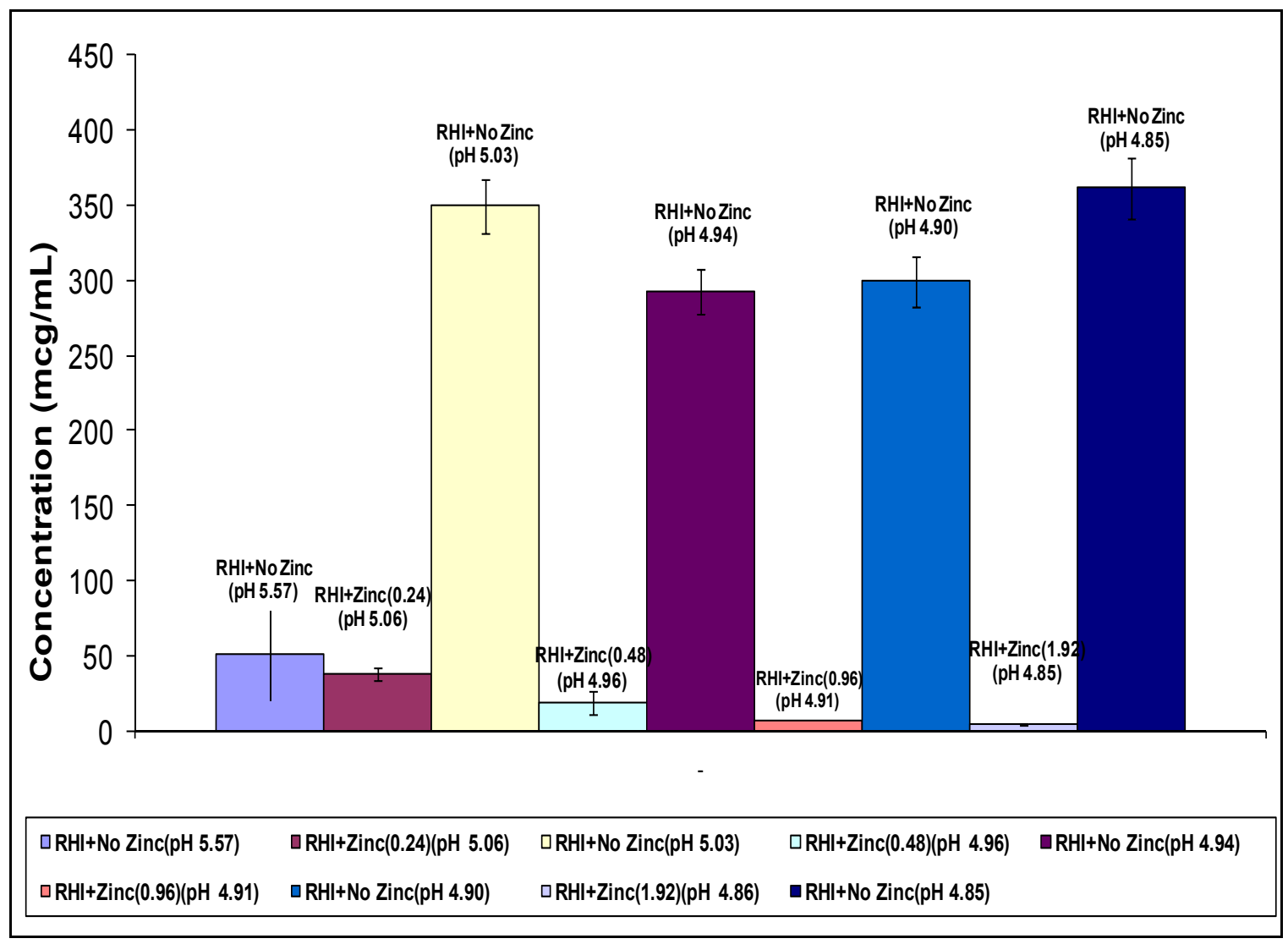

Figure 2-12 Concentration of insulin (RHI) in the supernatant obtained from formulations of insulin alone at different $\mathrm{pH}$, or formulations of insulin with varying concentrations zinc sulfate 


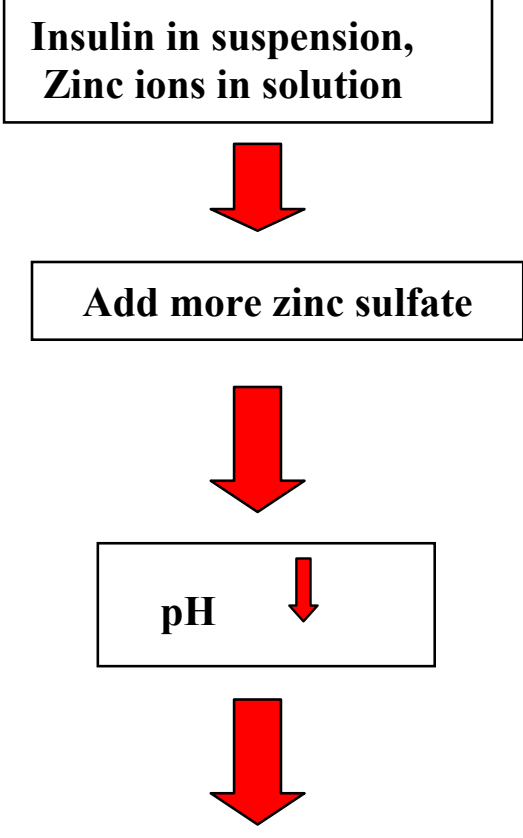

\section{More insulin solubilizes}

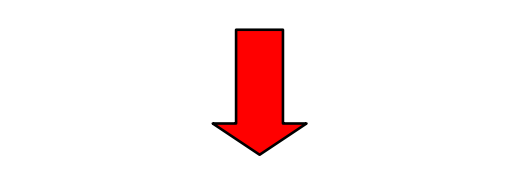

More hexamers formed

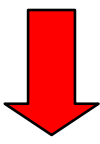

\section{Slow release of insulin}

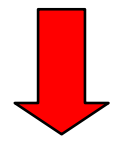

\section{Extended in vivo profile}

Figure 2-13 Possible mechanism of extended in vivo effect of formulations of insulin with varying concentrations zinc sulfate 


\section{CHAPTER 3. CONTROLLED RELEASE OF INSULIN FROM NOVEL BIODEGRADABLE INJECTABLE GELS}

\subsection{Introduction}

Diabetes mellitus is a chronic disorder characterized by hyperglycemia. It is recognized as a worldwide epidemic by the WHO. The disease may be further classified as type- 1 and type- 2 . Type- 1 diabetes mellitus patients can survive only by regular administration of insulin, whereas type- 2 diabetes mellitus patients can be treated with oral hypoglycemic agents and some other new classes of anti diabetic drugs. However, type- 2 patients may also need insulin therapy at some point. Hence, insulin is pivotal in treating diabetes (242-244).

For successful insulin therapy, daily subcutaneous injections of insulin are required. Subcutaneous insulin has been satisfactory in terms of efficacy in majority of cases. However the use of these subcutaneous injections may cause hyperinsulinemia which may lead to hypoglycemia and diabetic micro and macro angiopathy. Further, these subcutaneous injections of insulin are also associated with pain, inconvenience, physiological stress, high cost, difficulty in handling insulin, localized insulin deposition leading to local hypertrophy and fat deposition at site of injection. Most insulin regimens require multiple daily injections which are associated with poor patient compliance. Reduction in number of insulin injections can significantly improve efficacy of insulin therapy $(6,18)$.

Insulin interacts with zinc ions to form hexamers. In the presence of zinc, these hexamers further get stabilized. When these hexamers are injected via subcutaneous route, they form a depot at the site of injection because of their inability to cross the capillary barriers. To get absorbed into the blood stream, these hexamers have to dissociate first into dimers and then to monomers. This leads to a delayed and extended effect of insulin in presence of zinc $(124,238,245)$.

Various controlled release forms of insulin like microspheres, implants, gels, liposomes, and stereocomplexes have been investigated as extended release dosage forms of insulin. A novel injectable biodegradable gel system for controlled release drug delivery has been designed and characterized in our laboratory. It consists of a biodegradable polymer, PLGA, biocompatible plasticizer(s) and an active pharmaceutical ingredient (API). Chen et al. reported use of these gel systems for extended delivery of insulin for approximately one week (113). Chen et al. used ultralente crystals extracted from a commercial formulation (113). They also showed that adding a water-insoluble salt of zinc, zinc carbonate, further slowed down the release profile from these gels.

This work is continuation of the work done by Chen et al. and the aim of this study was to design insulin gel formulations, which could provide constant blood glucose lowering effect of the released insulin in diabetic rats for one week or longer, after a single subcutaneous injection. However in this part of study we intend to study the 
incorporation of water-soluble salt, zinc sulfate, and its effect on prolongation of blood glucose lowering effect of regular human insulin, released from these gels.

\subsection{Materials and methods}

\subsubsection{Materials}

Insulin (RHI) powder (26.9 IU/mg, 0.4\% zinc) used in this study was purchased from Diosynth France S.A. (Usine St. Charles, France). The polylactic-co-glycolic acid (PLGA) used in this study was Medisorb ${ }^{\circledR} 5050$ DL 1A (i.v. 0.09 dL/g). It was donated by Alkermers Inc. (Cambridge, MA). According to the manufacturer's technical information, PLGA 5050 DL 1A has a degradation time of 1-2 weeks (246). This grade of PLGA was selected because it has the shortest degradation time. Zinc sulfate used in this study was of laboratory grade and was bought from Sigma Chemicals (St. Louis, MO). Acetyl triethyl citrate (ATEC) and triethyl citrate (TEC) used in this study were generous donations from Morflex Inc. (Greensboro, NC). Male ZDF (fa/fa) rats weighing 300-400 grams used for the in vivo studies were received as donations from the Charles River Laboratories, Inc., (Wilmington, MA). Blood glucose was measured using Bayer Ascensia ${ }^{\circledR}$ Breeze blood glucose meter (Bayer Corporation, Elkhart, IN).

\subsubsection{Methods}

3.2.2.1 Preparation of insulin-zinc sulfate co-precipitates. An appropriate quantity of insulin (RHI) powder was suspended in normal saline to make insulin suspensions. Table 3-1 shows the composition of insulin formulations containing varying concentrations of zinc sulfate. The ingredients were weighed into a glass vial and water was added to bring the final weight to approximately 60 grams. Any lumps, if present, were broken using a stainless steel spatula. These formulations were kept at room temperature overnight (approximately 16 hours). The next day, the supernatant of the formulation was carefully removed and the precipitates were dried under vacuum at room temperature for 24 hours. The next day, the supernatant of the formulation was carefully removed and the precipitates were dried under vacuum at room temperature for 24 hours. The dried insulin-zinc sulfate co-precipitates were checked for any lumps, which if present were carefully triturated using a stainless steel spatula. Appropriate amounts of these insulin-zinc sulfate co-precipitates were loaded into the blank gel as shown in the Table 3-2 one day prior to subcutaneous administration to the ZDF rats. Zinc concentrations in the insulin-zinc precipitates were determined by atomic absorption spectrophotometer.

3.2.2.2 Fabrication of the gels. The gel formulations were prepared in two steps: dissolution of polymer in the plasticizer(s) to obtain a blank gel and loading of insulin alone or insulin zinc sulfate co-precipitates or insulin and zinc sulfate separately into the blank gel. The polymer, PLGA was allowed to come to room temperature in vacuum desiccators after it was taken out of the freezer. 
Table 3-1 Formulations of insulin suspensions

\begin{tabular}{ccccccc}
\hline $\begin{array}{c}\text { Formulation } \\
\#\end{array}$ & $\begin{array}{c}\text { Insulin } \\
\mathbf{( m g )}\end{array}$ & $\begin{array}{c}\mathbf{Z n ~ S O 4} \\
\mathbf{( \mathbf { m g } )}\end{array}$ & $\begin{array}{c}\mathbf{N a C l} \\
\mathbf{( m g )}\end{array}$ & $\begin{array}{c}\text { Adjust total } \\
\text { weight with } \\
\mathbf{H} \mathbf{2} \mathbf{( g )}\end{array}$ & $\begin{array}{c}\text { Amount of } \\
\text { zinc in the } \\
\text { formulation } \\
\text { (mg/g) }\end{array}$ & $\begin{array}{c}\text { Molar } \\
\text { ratio of } \\
\text { insulin } \\
\text { to zinc }\end{array}$ \\
\hline OA-3-3 & 90 & 63 & 530 & 60 & 0.24 & $1: 14$ \\
OA-3-4 & 90 & 127 & 521 & 60 & 0.48 & $1: 28$ \\
OA-3-5 & 90 & 253 & 501 & 60 & 0.96 & $1: 56$ \\
OA-3-6 & 90 & 507 & 464 & 60 & 1.92 & $1: 112$ \\
\hline
\end{tabular}


Table 3-2 Formulation composition of PLGA gel containing insulin and zinc

\begin{tabular}{|c|c|c|c|c|c|c|}
\hline $\begin{array}{c}\text { Formulation } \\
\#\end{array}$ & $\begin{array}{c}\text { ATEC } \\
(\%)\end{array}$ & $\begin{array}{r}\text { TEC } \\
(\%)\end{array}$ & $\begin{array}{c}\text { Insulin }+ \\
\mathrm{ZnSO}_{4} \\
\text { co- } \\
\text { precipitate } \\
(\%)\end{array}$ & $\begin{array}{l}\text { Polymer } \\
\text { (\%) }\end{array}$ & $\begin{array}{c}\text { Insulin }+ \\
\mathrm{ZnSO}_{4} \\
\text { co- } \\
\text { Precipitate } \\
\text { of } \\
\text { formulation }\end{array}$ & $\begin{array}{c}\text { Actual } \\
\text { Ratio } \\
\text { Insulin: } \\
\text { zinc }\end{array}$ \\
\hline OA-3-7 & 68.25 & 22.75 & 4 & 5 & OA-3-3 & $1: 14$ \\
\hline OA-3-8 & 68.25 & 22.75 & 4 & 5 & OA-3-4 & $1: 28$ \\
\hline OA-3-9 & 68.25 & 22.75 & 4 & 5 & OA-3-5 & $1: 56$ \\
\hline OA-3-10 & 68.25 & 22.75 & 4 & 5 & OA-3-6 & $1: 112$ \\
\hline
\end{tabular}


The desired amount of plasticizer(s) was weighed into the scintillation vial containing the polymer. A plastic screw cap with aluminum liner was used to seal the vial. The vial was then vortexed on a vortex mixer to disperse the polymer particles in the plasticizer(s). The vial was placed and in a Lab-Line orbital shaker (Model 3527, Labline, Melrose Park, IL) maintained at $37^{\circ} \mathrm{C}$ and shaken at $150 \mathrm{rpm}$. It took 2 to 3 days for the polymer to completely dissolve.

The resulting blank gel was stored in a vacuum desiccator at room temperature until further use (typically 1 to 2 days). For preparing insulin-loaded gel, a desired amount of blank gel was transferred to a new glass scintillation vial, and an appropriate amount of either pure insulin particles (Table 3-3) or insulin-zinc sulfate co-precipitates (Table 3-4) was accurately weighed and transferred into the blank gel.

\subsubsection{Preparation of physical mixtures of insulin and zinc sulfate. In the} formulations containing insulin and zinc sulfate, the zinc sulfate was first passed through sieve number $400(37.5 \mu \mathrm{m})$. An appropriate amount of the sieved zinc sulfate was weighed and mixed with insulin by the geometric dilution method. The resulting mixture of insulin and zinc sulfate was loaded into the blank gels. A stainless steel spatula was used to stir the mixture. Any visible agglomerates of insulin and/or zinc sulfate were broken using the spatula. Mixing was continued until a uniform mixture of insulin and zinc sulfate in the gel was produced.

3.2.2.3 Pharmacodynamic studies of in ZDF rats. All the experimental procedures for the animal studies were approved by the University of Tennessee Health Science Center's Animal Resources Advisory Committee (Protocol 1622). The ZDF rats (Charles River Laboratories, Wilmington, MA) were at least 16 weeks old and weighed approximately 300 to $400 \mathrm{~g}$. Hyperglycemia (> $400 \mathrm{mg} / \mathrm{dL}$ ) in the ZDF rats was confirmed by blood glucose measurements.

The rats were group-housed ( 3 per cage) in the animal facility under a 12-hour light/dark cycle. They had access to food and water ad libitum throughout the studies. Before the animals were shipped from Charles River Laboratories, they were fed Purina $\operatorname{diet}$ \#5008 until they fully developed diabetes (12 weeks old). The animals were on regular laboratory rodent food in our facility. The rats were acclimated to the environment for at least three days before they were used in the studies.

On each day of the study, the ZDF rats were transferred to the procedure room. The rats were randomly divided into groups, with 4 to 6 animals per group. Each group, except the control group, received one dose of an insulin formulation. The control group did not receive any insulin injection. Before injection, each rat was placed in an induction chamber and anesthetized by isoflurane (Halocarbon Product Corporation, River Edge, NJ) using a SurgiVet/Anesco ventilator (Waukesha, WI). Body weight of the animal was recorded to determine the volume of the insulin formulation to be injected. Blood glucose was measured using Bayer Ascensia ${ }^{\circledR}$ DEX $^{\circledR} 2$ or Ascensia Breeze blood glucose meter (Bayer Corporation, Elkhart, IN). The dose of insulin formulation was injected subcutaneously at the dorsal neck of the rat using a $1 \mathrm{~mL}$ syringe with an attached 23 
Table 3-3 Formulation composition of PLGA gel containing insulin and zinc sulfate physical mixtures

\begin{tabular}{ccccccc}
\hline $\begin{array}{c}\text { Formulation } \\
\#\end{array}$ & $\begin{array}{c}\text { ATEC } \\
(\%)\end{array}$ & $\begin{array}{c}\text { TEC } \\
(\%)\end{array}$ & $\begin{array}{c}\text { Insulin } \\
(\%)\end{array}$ & $\begin{array}{c}\text { Polymer } \\
(\%)\end{array}$ & $\begin{array}{c}\mathbf{Z n S O}_{4} \\
(\%)\end{array}$ & $\begin{array}{c}\text { Actual } \\
\text { ratio } \\
\text { insulin: } \\
\text { zinc }\end{array}$ \\
\hline OA-3-11 & 68.25 & 22.75 & 4 & 5 & 1 & $1: 14$ \\
OA-3-12 & 68.25 & 22.75 & 4 & 5 & 2 & $1: 28$ \\
OA-3-13 & 68.25 & 22.75 & 4 & 5 & 2.8 & $1: 14$ \\
OA-3-14 & 68.25 & 22.75 & 4 & 5 & 5.6 & $1: 28$ \\
\hline
\end{tabular}


Table 3-4 Zinc contents in insulin-zinc co-precipitates

\begin{tabular}{cccc}
\hline $\begin{array}{c}\text { Precipitate of } \\
\text { Formulation } \\
\#\end{array}$ & $\begin{array}{c}\text { Formulation } \\
\text { composition of } \\
\text { precipitate } \\
(\mathbf{m g} / \mathbf{g})\end{array}$ & $\begin{array}{c}\text { Amount zinc } \\
(\boldsymbol{\mu m}) / \text { insulin }(\mathbf{m g})\end{array}$ & $\begin{array}{c}\text { Ratio } \\
\text { insulin : zinc }\end{array}$ \\
\hline OA-3-3 & RHI-Zn-0.24 & $7.78 \pm 0.69$ & $06: 04.1$ \\
OA-3-4 & RHI-Zn-0.48 & $10.88 \pm 0.96$ & $06: 05.1$ \\
OA-3-5 & RHI-Zn-.96 & $13.77 \pm 2.77$ & $06: 07.2$ \\
OA-3-6 & RHI-Zn-1.92 & $11.67 \pm 3.82$ & $06: 06.1$ \\
\hline
\end{tabular}


gauge 1 inch needle (Franklin Lakes, NJ). After injection, the rats were returned to the cages and allowed to recover from anesthesia. At pre-determined time intervals, the rats were anesthetized by aforementioned method and their blood glucose concentrations were measured.

A plot of blood glucose concentrations versus time was generated from the data to describe the glucose lowering effect of insulin, from which information such as time of peak action and duration of action could be obtained.

\subsection{Results and discussion}

Pharmacodynamic studies of different gel formulations of insulin in ZDF rats: Two different gel formulations consisting of 5 and $10 \%$ PLGA were made by suspending insulin particles in the blank gels (Table 3-5). These insulin formulations were administered subcutaneously to ZDF rats to evaluate the biological effect of insulin released from these formulations. Specifically, the blood glucose concentrations and body weights of the rats were measured at periodic time intervals following the subcutaneous injection. Figure 3-1 shows changes in blood glucose concentrations of ZDF rats after a subcutaneous injection of PLGA (5 and 10\%) gel formulation loaded with $4 \%$ insulin (Formulations OA-3-1 and OA-3-2). It is evident from Figure 3-1 that the blood glucose concentrations decreased from approximately $554 \pm 29.4 \mathrm{mg} / \mathrm{dL}$ to $63 \pm 10.6 \mathrm{mg} / \mathrm{dL}$ within 6 hours after a subcutaneous injection of Formulation OA-3-1 (5\% PLGA) in the ZDF rats. The blood glucose concentrations were below $80 \mathrm{mg} / \mathrm{dL}$ in all the six rats, i.e. all the rats were hypoglycemic within six hours after injection. Further, the blood glucose concentrations of all the six rats were below $70 \mathrm{mg} / \mathrm{dL}$ within 12 hours after subcutaneous administration of the formulation. However, the blood glucose concentrations of all the six rats increased to normally high values of $443 \pm 55.7 \mathrm{mg} / \mathrm{dL}$ within 24 hour after subcutaneous administration of the formulation, and remained high thereafter. A similar blood glucose concentration profile was obtained after subcutaneous injection of formulation OA-3-2 (10\% PLGA). The blood glucose concentrations decreased from approximately $452 \pm 27.38 \mathrm{mg} / \mathrm{dL}$ to $92.5 \pm 4.9 \mathrm{mg} / \mathrm{dL}$ within 6 hours after a subcutaneous injection of the formulation.

The blood glucose concentrations further declined to below $75 \mathrm{mg} / \mathrm{dL}$ after 12 hours after administration of the formulation. As with $5 \%$ PLGA formulation, the blood glucose concentrations of all of the six rats increased to normally high values of $331 \pm$ $35.1 \mathrm{mg} / \mathrm{dL}, 24$ hours after administration of the formulation and remained high thereafter. It is evident from these results that these insulin formulations consisting of 5 and $10 \%$ PLGA release insulin at a very rapid rate, leading to hypoglycemia in all the rats. It is evident from these results that these insulin formulations consisting of 5 and $10 \%$ PLGA release insulin at a very rapid rate, leading to hypoglycemia in all the rats. Further, the blood glucose concentrations could not be maintained at low concentrations ( 80 to $100 \mathrm{mg} / \mathrm{dL}$ ) for a long period of time, and the all the rats were hyperglycemic within 24 hours after the subcutaneous injection of these formulations. 
Table 3-5 Formulation composition of PLGA gel of insulin

\begin{tabular}{ccccc}
\hline $\begin{array}{c}\text { Formulation } \\
\#\end{array}$ & $\begin{array}{c}\text { ATEC } \\
(\%)\end{array}$ & $\begin{array}{c}\text { TEC } \\
(\%)\end{array}$ & $\begin{array}{c}\text { Insulin } \\
(\%)\end{array}$ & $\begin{array}{c}\text { Polymer } \\
(\%)\end{array}$ \\
\hline OA-3-1 & 68.25 & 22.75 & 4 & 5 \\
OA-3-2 & 68.25 & 22.75 & 4 & 10 \\
\hline
\end{tabular}




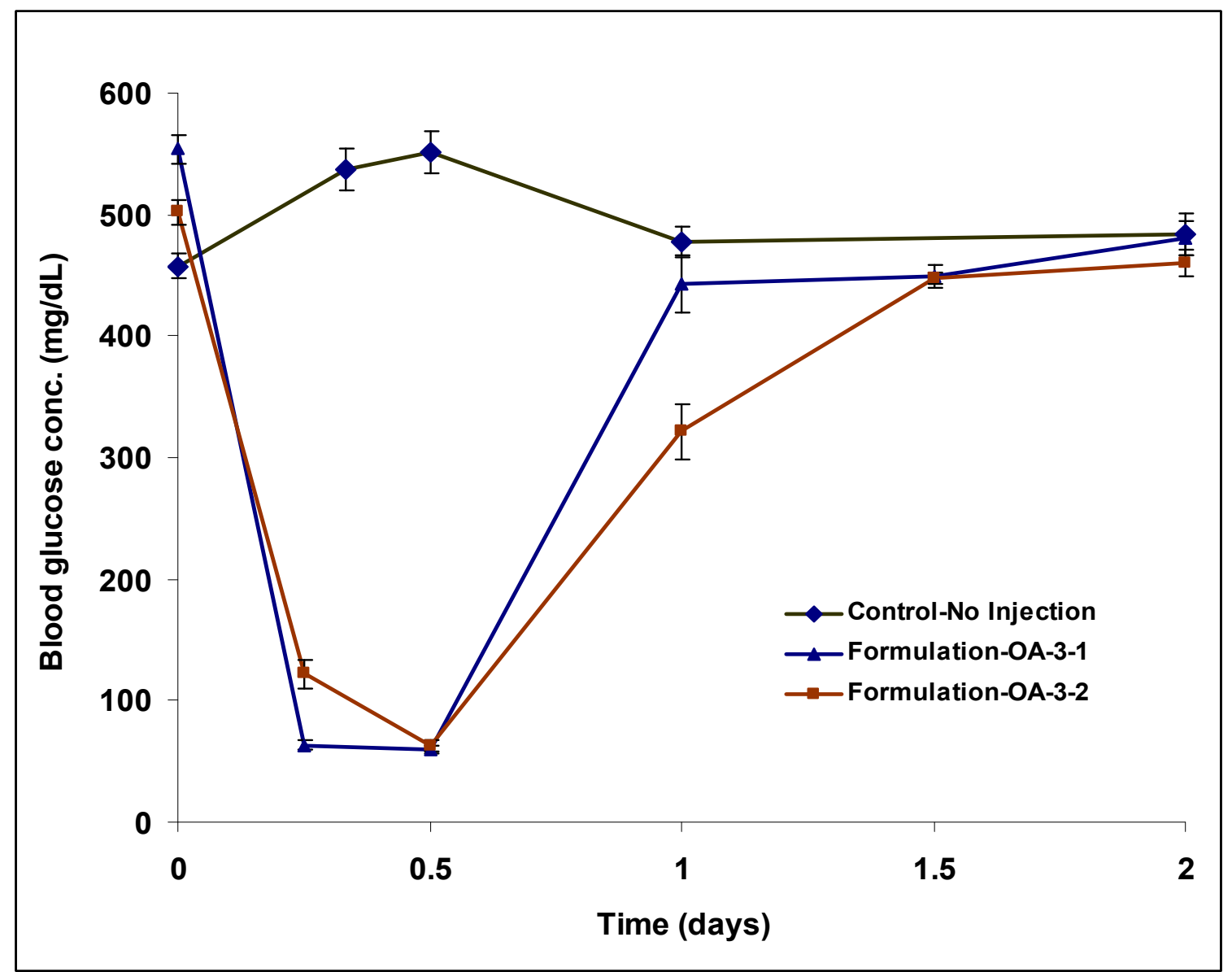

Figure 3-1 Changes in blood glucose concentrations of ZDF rats after a subcutaneous injection of PLGA (5\% and 10\%) gel formulations loaded with 4\% insulin (Formulations OA-3-1 and OA-3-2) (Mean \pm SEM, $n=5)$ 
Figure 3-2 shows changes in the body weights of ZDF rats after a subcutaneous injection of the PLGA (5 and 10\% ) gel formulation loaded with $4 \%$ insulin (Formulations OA-3-1 and OA-3-2). The body weights of these ZDF rats 24 hours after subcutaneous injection of the PLGA formulations (5 and 10\%) were higher than before the injection of the formulations. However, the body weights came back to approximate initial values within 48 hours after the injection of these formulations. This pharmacodynamic effect shown by the changes of the body weights after the subcutaneous injection is similar to the pharmacodynamic effect of the changes in the blood glucose concentrations. This shows that these formulations (Formulations OA-3-1 and OA-3-2) could not control the release of insulin for a prolonged period of time after a single subcutaneous injection in the ZDF rats, and the insulin effect lasted for less than 24 hours.

As shown in the previous study (Chapter-2) and reported by other groups, insulin zinc interaction can extend the in vivo duration of action of insulin (238). Further, as shown by Chen et al. (113) and Kim et al. (247), incorporation of water-insoluble zinc salt in a similar type of polymer based gels slowed down the insulin release and extended the in vivo duration of action of insulin. In this study, we purposed that incorporation of a water-soluble zinc salt, zinc sulfate, in these PLGA formulations of regular human insulin can prolong the blood glucose lowering effect of regular human insulin.

The insulin-zinc co-precipitates (Formulations OA-3-3, OA-3-4, OA-3-5 and OA3-6) were loaded in 5\% PLGA gels and four different formulations were obtained (Formulations OA-3-7, OA-3-8, OA-3-9 and OA-3-10). These formulations were injected subcutaneously in ZDF rats and change in blood glucose concentrations and body weights were monitored for a week. Figure 3-3 shows the changes in blood glucose concentrations of ZDF rats after a subcutaneous injection of 5\% PLGA gel formulation loaded with 4\% insulin alone (Formulation OA-3-1) or insulin-zinc sulfate co-precipitates (Formulations OA-3-7 and OA-3-8). It is evident from the figure that the blood glucose lowering effect of 5\% PLGA formulation consisting of insulin alone (Formulation: OA3-1) lasted less than 24 hours. After a subcutaneous injection of 5\% PLGA formulation (Formulation OA-3-7), consisting of insulin-zinc sulfate co-precipitates of Formulation OA-3-3 (0.24 mg of zinc /gram of formulation), the blood glucose concentrations.

The blood glucose concentrations remained below $100 \mathrm{mg} / \mathrm{dL}$ until 24 hours before increasing to approximately $200 \mathrm{mg} / \mathrm{dL}$ after 48 hours after the subcutaneous injection of the formulation. The blood glucose concentrations steadily increased above $350 \mathrm{mg} / \mathrm{dL}$ and remained above this concentration thereafter. In the case of 5\% PLGA formulation (Formulation OA-3-8), consisting of insulin-zinc co-precipitates of Formulation OA-3-4 (0.48 mg of zinc gram of formulation), the blood glucose concentrations decreased from approximately $426 \pm 15.9 \mathrm{mg} / \mathrm{dL}$ to $75 \pm 17.3 \mathrm{mg} / \mathrm{dL}$ within 12 hours after the subcutaneous injection. The blood glucose concentrations remained below $150 \mathrm{mg} / \mathrm{dL}$ for up to 3 days before increasing to approximately 330 $\mathrm{mg} / \mathrm{dL}$ after 4 days after the subcutaneous injection of the formulation. The blood glucose concentrations increased steadily above $350 \mathrm{mg} / \mathrm{dL}$ and remained above this concentration thereafter. This shows that when the insulin-zinc co-precipitates were 


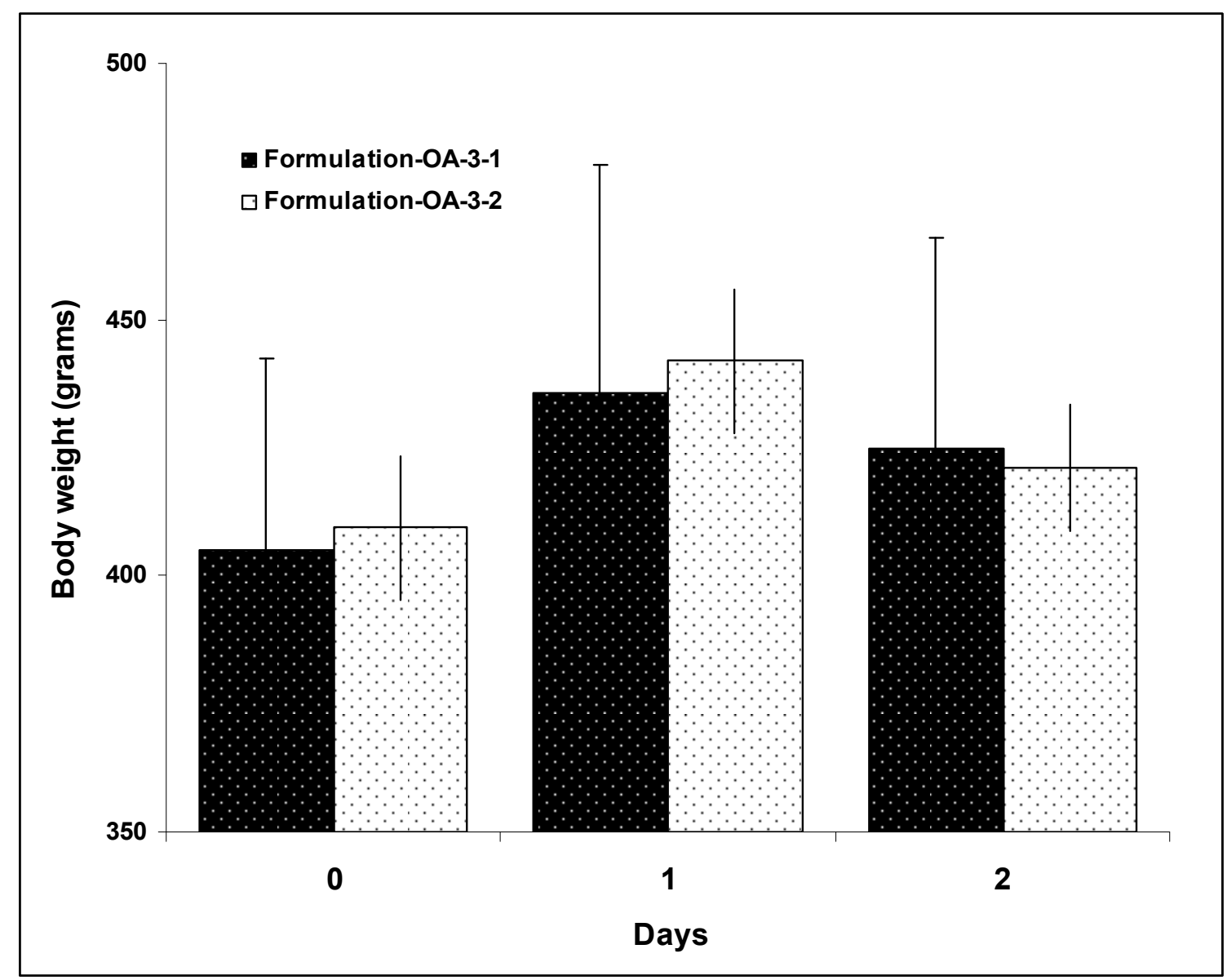

Figure 3-2 Changes in body weights of ZDF rats after subcutaneous injections of PLGA (5 and 10\%) gel formulations loaded with 4\% insulin (Formulations OA-3-1 and OA-3-2) (Mean \pm SEM, $n=5)$ 


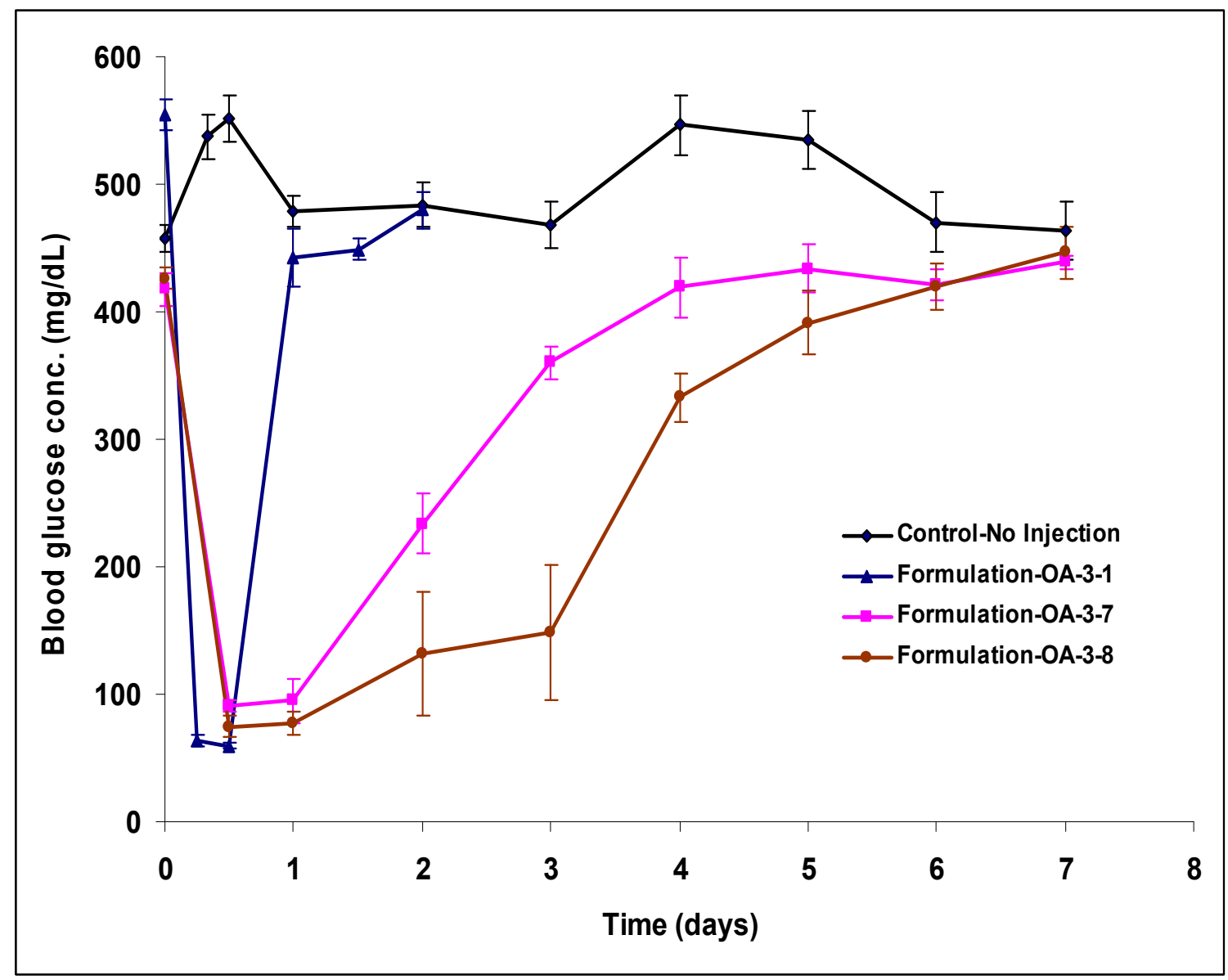

Figure 3-3 Changes in blood glucose concentrations of ZDF rats after subcutaneous injections of PLGA (5\%) gel formulations loaded with 4\% insulin alone (Formulation OA-3-1) or insulin-zinc co-precipitates (Formulations OA-3-7 and OA-3-8) (Mean \pm $\mathrm{SEM}, \mathrm{n}=5$ ) 
loaded in the 5\% PLGA gel formulations (Formulations OA-3-5 and OA-3-6) and injected subcutaneously in the ZDF rats, the blood glucose concentrations decreased slowly and remained at low concentrations (between 100 and $200 \mathrm{mg} / \mathrm{dL}$ ) for a longer period of time. Addition of zinc also reduced the chances of occurrence of hypoglycemia in the ZDF rats after the subcutaneous injection of these PLGA formulations containing insulin. Further, with increase in the zinc, the in vivo effect of insulin was extended from 1 day to up to 3 days.

Figure 3-4 shows the changes in blood glucose concentrations of ZDF rats after a subcutaneous injection of 5\% PLGA gel formulation loaded with 4\% insulin alone (Formulation OA-3-1) or insulin-zinc co-precipitates (Formulations OA-3-9 and OA-3$10)$.

Figure 3-4 shows the changes in blood glucose concentrations of ZDF rats after a subcutaneous injection of 5\% PLGA gel formulation loaded with $4 \%$ insulin alone (Formulation OA-3-1) or insulin-zinc co-precipitates (Formulations OA-3-9 and OA-310). After subcutaneous injection of 5\% PLGA formulation (Formulation OA-3-9), consisting of insulin-zinc co-precipitates of Formulation OA-3-5 (0.96 mg of zinc /gram of formulation), the blood glucose concentrations decreased from approximately $492 \pm$ $29.3 \mathrm{mg} / \mathrm{dL}$ to $105 \pm 19.2 \mathrm{mg} / \mathrm{dL}$ within 12 hours after the subcutaneous injection. The blood glucose concentrations remained below $150 \mathrm{mg} / \mathrm{dL}$ for up to 2 days before increasing to approximately $300 \mathrm{mg} / \mathrm{dL}$ after 3 days after the subcutaneous injection of the formulation. The blood glucose concentrations increased steadily above $350 \mathrm{mg} / \mathrm{dL}$ and remained above this concentration thereafter. In the case of 5\% PLGA formulation (Formulation OA-3-10 ) consisting of insulin- zinc co-precipitates of Formulation (Formulation OA-3-6 with $1.92 \mathrm{mg}$ of zinc /gram of formulation), the blood glucose concentrations decreased slowly from approximately $467 \pm 51.5 \mathrm{mg} / \mathrm{dL}$ to $99.8 \pm 17.5$ $\mathrm{mg} / \mathrm{dL}$ within 24 hours after the subcutaneous injection. The blood glucose concentrations remained below $175 \mathrm{mg} / \mathrm{dL}$ for up to 3 days before increasing to approximately $300 \mathrm{mg} / \mathrm{dL}$ after 4 days after the subcutaneous injection of the formulation.

The blood glucose concentrations increased steadily above $350 \mathrm{mg} / \mathrm{dL}$ and remained above this concentration thereafter. This change in blood glucose concentrations shows that the effect of insulin does not last longer than 3 days after the subcutaneous injection of these 5\% PLGA formulations containing insulin-zinc coprecipitates. Further, there was an increase in the duration of blood glucose lowering effect of insulin from Formulation OA-3-8 (5\% PLGA gel containing 4\% insulin-zinc coprecipitate with $0.48 \mathrm{mg}$ of zinc/ gram of formulation) as compared to Formulation OA3-7 (5\% PLGA gel containing 4\% insulin-zinc co-precipitate with $0.24 \mathrm{mg}$ zinc / gram of formulation) and Formulation OA-3-1 (5\% PLGA gel containing 4\% insulin). However, there was no further increase in the in vivo duration of insulin when 5\% PLGA gel ormulations loaded with insulin-zinc co-precipitates having more zinc sulfate (obtained from Formulation OA-3-5 with $0.96 \mathrm{mg}$ zinc/ gram of formulation and Formulation OA3-6 with with $1.92 \mathrm{mg}$ zinc / gram of formulation), were subcutaneously injected in the rats. 


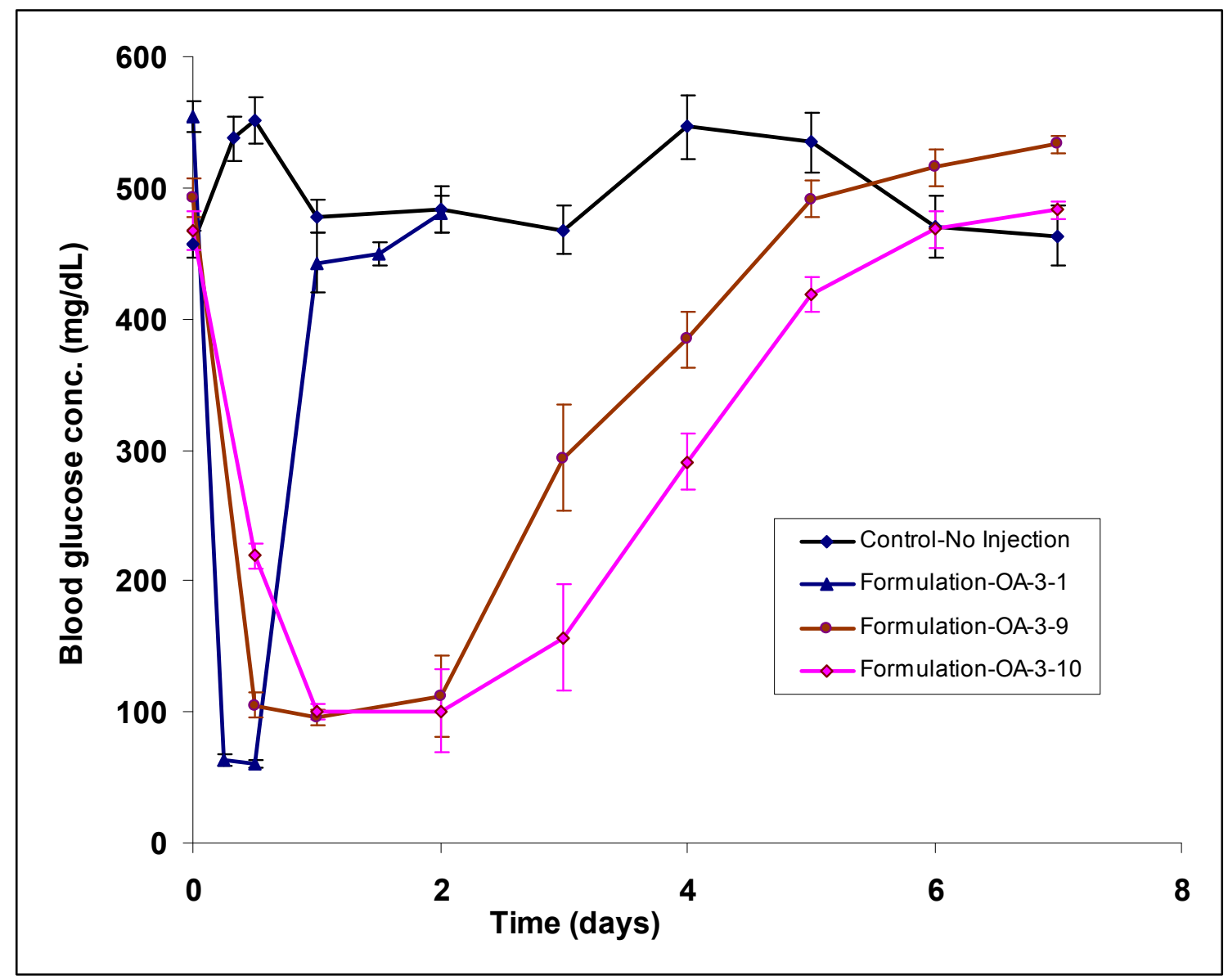

Figure 3-4 Changes in blood glucose concentrations of ZDF rats after subcutaneous injections of PLGA (5\%) gel formulations loaded with 4\% insulin alone (Formulation OA-3-1) or insulin-zinc co-precipitates (Formulations OA-3-9 and OA-3-10) (Mean \pm $\mathrm{SEM}, \mathrm{n}=5$ ) 
After subcutaneous injections of 5 and 10\% PLGA formulations of insulin (Figure 3-1) all the rats went to hypoglycemia within 6 hours. However, as the insulin-zinc coprecipitates loaded gels were subcutaneously injected in rats, the number of rats going into hypoglycemia decreased substantially. The blood glucose concentrations also decreased slowly after the subcutaneous injection of the 5\% PLGA formulations of insulin-zinc co-precipitates.

Figure 3-5 shows the changes in the body weights of ZDF rats after a subcutaneous injections of 5\% PLGA gel formulations loaded with 4\% insulin-zinc coprecipitates (Formulations OA-3-7, OA-3-8, OA-3-9 and OA-3-10). It is evident from the figure that the body weights of ZDF rats increased 24 hours after the subcutaneous injections. However, in the case of Formulation OA-3-7, a very rapid weight gain was observed on day 1, after which it slowly declined. In the case of other formulations, the body weights of ZDF rats increased on day 1 and remained steady for up to day 3 followed by a slow decline after the subcutaneous injections. This change in body weights also showed that the effect of insulin does not last longer than 3 days after subcutaneous injection of these 5\% PLGA formulations containing insulin-zinc coprecipitates. Since the blood glucose lowering effect of these 5\% PLGA formulations containing insulin-zinc co-precipitates could not be extended for more than three days, the actual zinc content of these insulin-zinc co-precipitates was determined using atomic absorption spectrophotometer. As shown in Table 3-5, the actual zinc content of these coprecipitates was much lower. Two zinc ions are required to interact with six molecules of insulin to form a hexamer. These insulin-zinc co-precipitates have 4 to 7 zinc ions per six insulin molecules, which may be sufficient to transform all the insulin molecules into hexamers. Therefore, the initial blood glucose lowering effect was slow in formulations containing insulin-zinc co-precipitates. However, after subcutaneous injections, these coprecipitates were exposed to interstitial fluid, which dissolved and quickly removed the zinc ions from the injection site. Furthermore, the zinc ions present in these coprecipitates were not in excess to extend the effect of insulin for a long time.

To increase the amount of zinc in the formulations, zinc sulfate was directly loaded in the PLGA gels. Figure 3-6 shows the change in blood glucose concentrations in ZDF rats after subcutaneous injections of insulin loaded in 5\% PLGA gel formulations with different concentrations of zinc sulfate (Formulations OA-3-11 and OA-3-12 containing 1 and 2\% zinc sulfate, respectively). Formulation OA-3-1 prepared with 5\% PLGA and having no zinc sulfate in it, showed a rapid decline in the blood glucose concentrations; reaching below $75 \mathrm{mg} / \mathrm{dL}$ in 6 hours. The blood glucose concentrations remained below $75 \mathrm{mg} / \mathrm{dL}$ for up to 12 hours, before climbing back to the normally high concentrations $350 \mathrm{mg} / \mathrm{dL}$ within 24 hours.

Formulations OA-3-11 and OA-3-12 containing 1 and $2 \%$ of zinc sulfate respectively, were prepared with 5\% PLGA. Formulation OA-3-11 (containing 1\% zinc sulfate) showed a slow decline in the blood glucose concentrations, reaching below 200 $\mathrm{mg} / \mathrm{dL}$ in 12 hours and below $75 \mathrm{mg} / \mathrm{dL}$ in 24 hours. The blood glucose concentrations remained below $100 \mathrm{mg} / \mathrm{dL}$ for 3 days before slowly climbing up to $300 \mathrm{mg} / \mathrm{dL}$ concentrations at 5 days and remained above this concentration thereafter. 


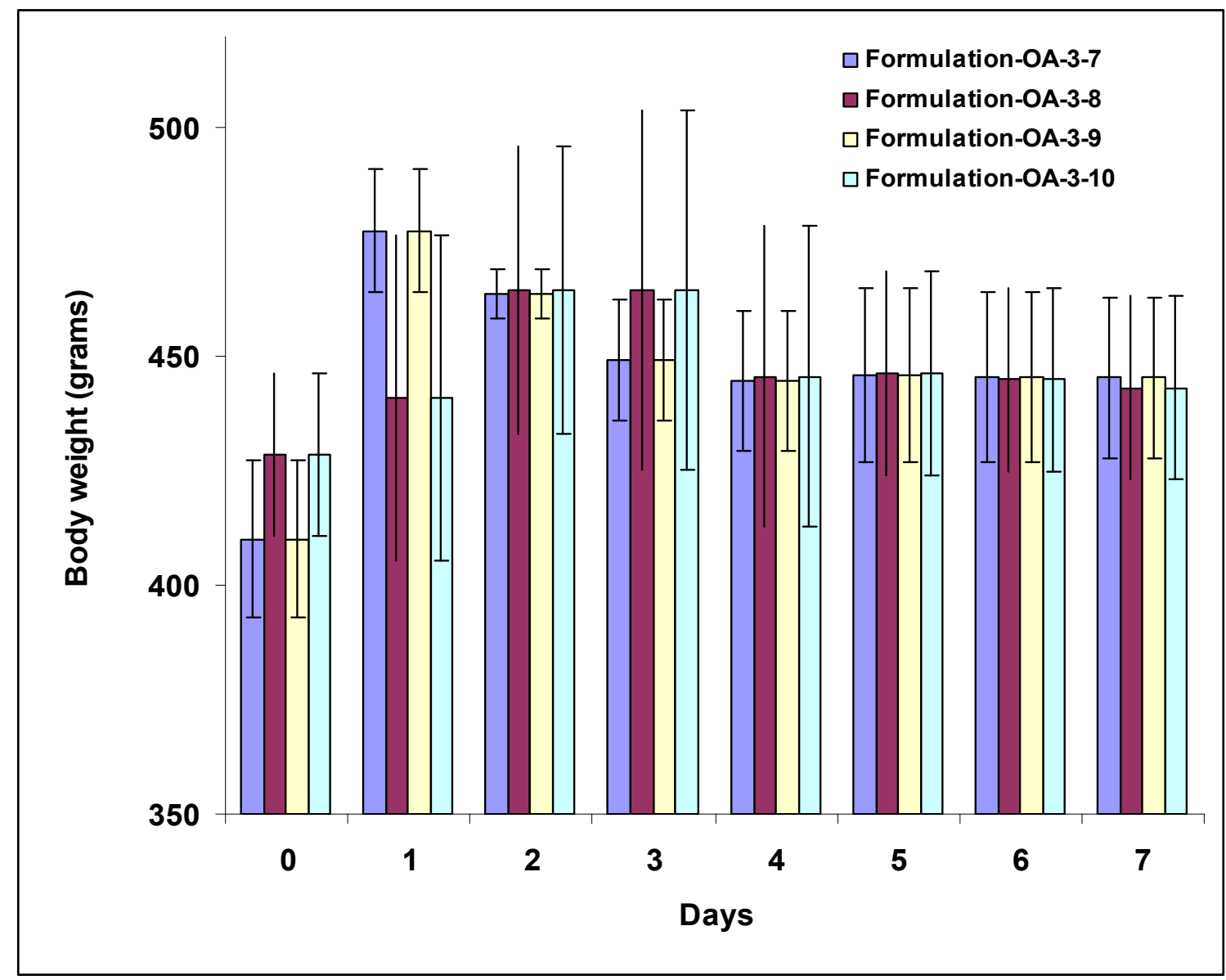

Figure 3-5 Changes in body weights of ZDF rats after subcutaneous injections of PLGA (5\%) gel formulations loaded with 4\% insulin-zinc co-precipitates (Formulations OA-3-7, OA-3-8, OA-3-9 and OA-3-10) (Mean \pm SEM, $n=5)$ 


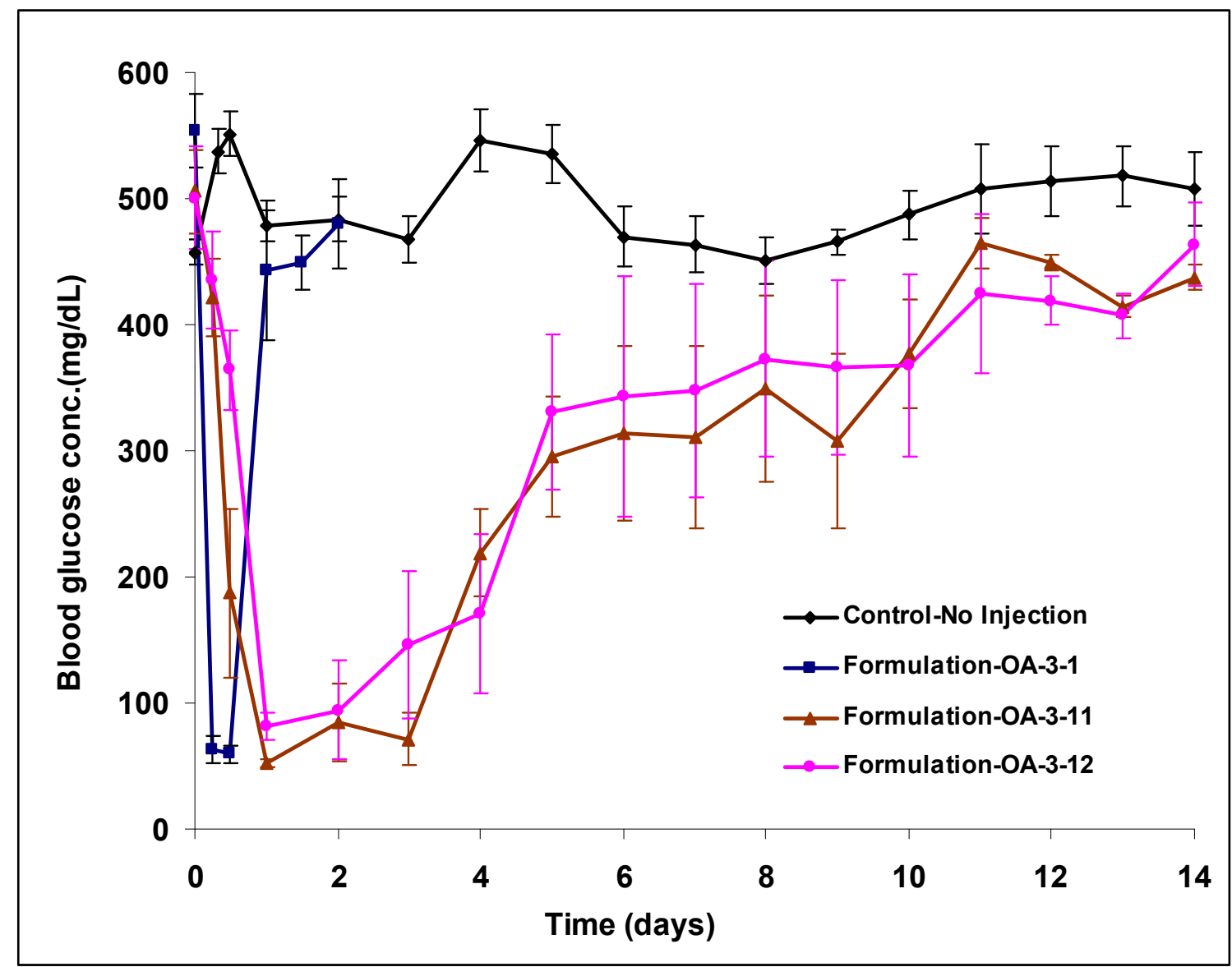

Figure 3-6 Changes in blood glucose concentrations of ZDF rats after subcutaneous injections of insulin alone (Formulations OA-3-1) or physical mixtures of insulin (4\%) and zinc sulfate ( 1 or $2 \%$ ) loaded in PLGA $(5 \%)$ gel formulations (Formulations OA-311 and $\mathrm{OA}-3-12)($ Mean $\pm \mathrm{SEM}, \mathrm{n}=5)$ 
Formulation OA-3-12 (containing 2\% zinc sulfate) showed a similar pattern as that of Formulation OA-3-11. When the zinc sulfate increased in the formulation, the blood glucose concentrations declined below $100 \mathrm{mg} / \mathrm{dL}$ even more slowly in 24 hours. The blood glucose concentrations remained below $200 \mathrm{mg} / \mathrm{dL}$ for 4 days before slowly climbing up to $300 \mathrm{mg} / \mathrm{dL}$ concentrations at 5 days and remained above this concentration thereafter. Thus, with addition of zinc sulfate in the 5\% PLGA formulations, the blood glucose concentrations decreased at a slower rate. However, even with addition of $2 \%$ zinc sulfate, the blood glucose concentrations remained below 200 $\mathrm{mg} / \mathrm{dL}$ only for 4 days. To investigate the effect of even higher concentrations of zinc sulfate, the amount of zinc sulfate was increased to 2.8 and $5.4 \%$ in the $5 \%$ PLGA gel formulations. Figure 3-7 shows the change in blood glucose concentrations in ZDF rats after subcutaneous injections of insulin loaded in 5\% PLGA gels with higher concentrations of zinc sulfate (2.8 and 5.4\%). Formulations OA-3-13 and OA-3-14 containing 2.8 and $5.6 \%$ of zinc sulfate, respectively were prepared with 5\% PLGA. Formulation OA-3-13 also showed a slow decline in the blood glucose concentrations, reaching below $100 \mathrm{mg} / \mathrm{dL}$ in 24 hours.

Formulation OA-3-13 also showed a slow decline in the blood glucose concentrations, reaching below $100 \mathrm{mg} / \mathrm{dL}$ in 24 hours. The blood glucose concentrations remained between 100 to $250 \mathrm{mg} / \mathrm{dL}$ for 9 days before slowly climbing up to $300 \mathrm{mg} / \mathrm{dL}$ concentrations at 10 days. The blood glucose concentrations remained above $300 \mathrm{mg} / \mathrm{dL}$ thereafter. Formulation OA-3-14 (containing 5.6\% zinc sulfate) showed a similar pattern as that of Formulation OA-3-13. However, the decline in blood glucose concentrations was slower (reaching below $100 \mathrm{mg} / \mathrm{dL}$ in 2 days) and lasted slightly longer after a subcutaneous injection of Formulation OA-3-14 compared to that of Formulation OA-313. Hence, the effect of insulin on the blood glucose concentrations was substantially increased with increasing zinc concentrations in the insulin-loaded gel formulations.

Figure 3-8 shows changes in body weights of ZDF rats after subcutaneous injections of 5\% PLGA gel formulations loaded with 4\% insulin and zinc sulfate mixture (Formulations OA-3-11, OA-3-12, OA-3-13 and OA-3-14). The changes in body weights of the rats repeated the pharmacodynamic trend that had been observed with the change in blood glucose concentrations after subcutaneous injections of these formulations.

\subsection{Conclusions}

A biodegradable injectable gel formulation prepared with zinc salts was able to sustain the release of insulin for up to 8 to 10 days following a single subcutaneous injection. The released insulin from the gel formulations was able to suppress the high blood glucose concentrations for the same period of time in the ZDF rats. However, a biodegradable injectable gel formulation of human insulin without zinc salts could maintain the blood glucose concentrations at low concentrations only for one day. 


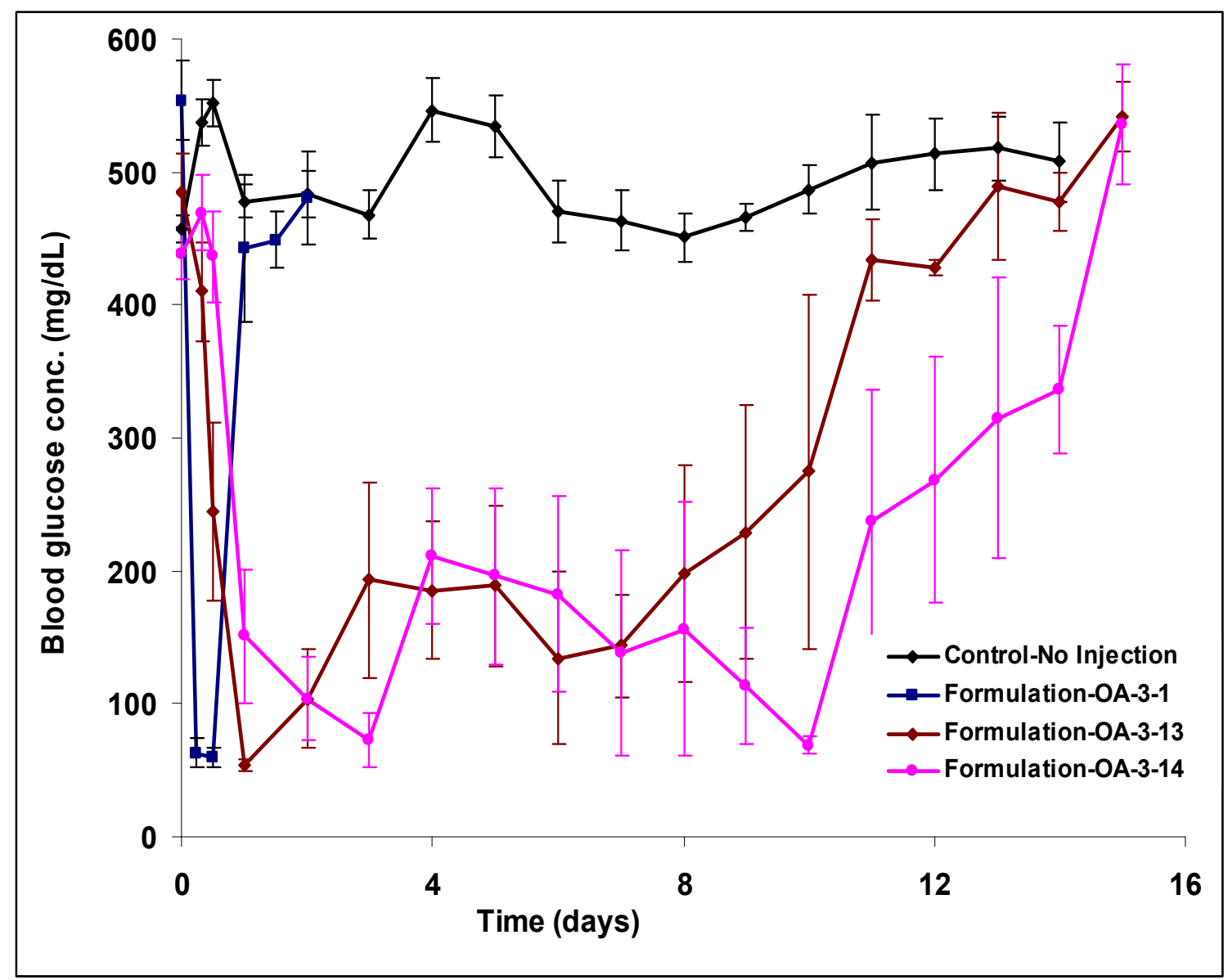

Figure 3-7 Changes in blood glucose concentrations of ZDF rats after subcutaneous injections of insulin loaded in PLGA (5\%) gel formulations with different concentrations of zinc sulfate (2.8\% and 5.4\%) (Formulations OA-3-13 and OA-3-14) (Mean \pm SEM, $n$ $=5)$ 


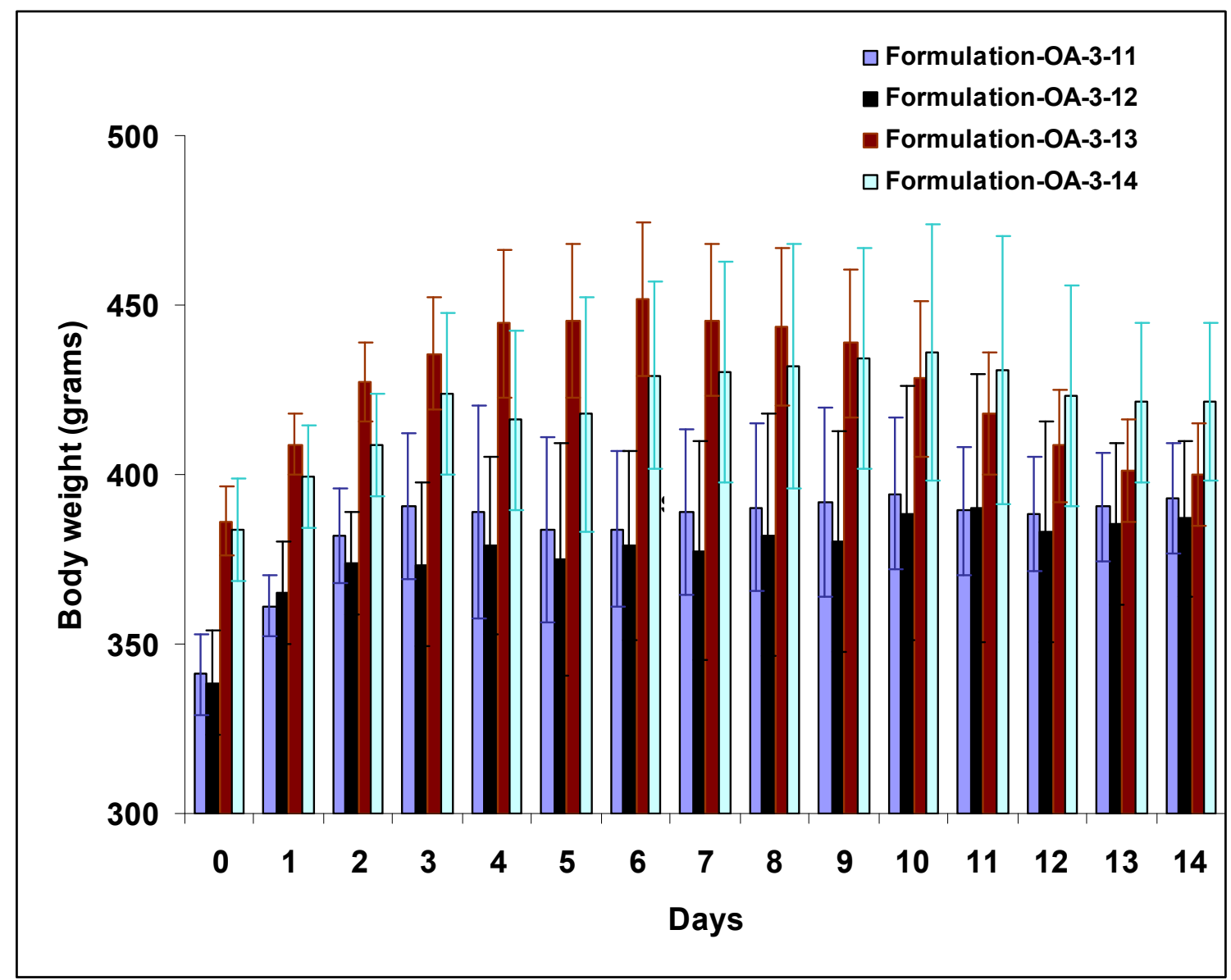

Figure 3-8 Changes in body weights of ZDF rats after subcutaneous injections of PLGA (5\%) gel formulations loaded with 4\% insulin and zinc sulfate mixtures (Formulations OA-3-11, OA-3-12, OA-3-13 and OA-3-14) $(\mathrm{Mean} \pm \mathrm{SEM}, \mathrm{n}=5$ ) 


\section{CHAPTER 4. CONTROLLED RELEASE OF MODIFIED INSULIN GLARGINE FROM NOVEL BIODEGRADABLE INJECTABLE GELS}

\subsection{Introduction}

Diabetes mellitus is a chronic metabolic disorder characterized by hyperglycemia, which may be due to deficiency or ineffectiveness of metabolic hormone insulin produced by Langharan's beta cells of pancreas. The form of diabetes characterized by absolute insulin deficiency is known as type-1. The most prevalent form of diabetes is type- 2 diabetes mellitus. It accounts for $90 \%$ of the cases and is due to ineffectiveness of insulin produced by the pancreas $(6,18,242)$.

The major objective of diabetes treatment is to achieve normoglycemia by maintaining appropriate concentrations of insulin in the blood throughout the day. Insulin has been complexed with protamine or zinc to delay and extend the release in vivo and prolong its effect for one day. However, these formulations have been associated with side effects such as hypoglycemia, and inter and intra-subject variability in absorption, and shorter duration of action than expected, thus leading to poor management of diabetes $(6,18,248)$.

For intensive blood glucose control in people suffering from type-1 and type- 2 diabetes, supply of basal insulin (a steady, low concentration of insulin that is constantly present in the circulation to cover post-prandial and overnight fasting periods) is very important. Protracted acting insulin preparations supply this basal concentration in clinical settings $(128,249,250)$. One of the formulations that maintain basal concentration insulin is modified insulin called insulin glargine. It has been altered at two different positions: in A-chain, at position 21, where the asparagine has been substituted with glycine, thus imparting more resistance to deamidation in the acidic environment and providing more stability. The $\mathrm{C}$ - terminus of the $\mathrm{B}$-chain has been elongated by the addition of two arginine molecules. This addition of two positive charges by the addition of arginines shifted the isoelectric point of the modified insulin glargine from $\mathrm{pH} 5.4$ to 6.7. These modifications have made it possible to formulate insulin glargine into slightly acid stable solution, which is easy to inject and improves dose reproducibility. This insulin glargine solution forms stable hexamers which precipitate at neutral $\mathrm{pH}$ at the subcutaneous site of injection, thus forming a depot of microprecipitates of the modified insulin glargine, which then dissolve at a steady rate for a prolonged period of time (128, 249, 251).

Insulin and modified insulin like glargine interact with zinc ions to form hexamers. In the presence of zinc, these hexamers get further stabilized. When these hexamers are injected via subcutaneous route, they form a depot at the site of injection because of their inability to cross the capillary barriers. To get absorbed into the blood stream, these hexamers have to dissociate first into dimers and then to monomers. This leads to a delayed and extended effect of insulin and modified insulin glargine. 
A novel biodegradable injectable gel system for controlled release drug delivery has been designed and characterized in our laboratory. It consists of a biodegradable polymer, PLGA, biocompatible plasticizer(s) and an active pharmaceutical ingredient (API). To date, several molecules, including antibiotics, opiate analgesics and narcotic antagonists, and insulin have been successfully incorporated into the gel system, and controlled release of the aforementioned APIs have been achieved in our laboratory.

Therefore, the aim of this study was to develop an injectable biodegradable gel system which can be used to control the release of insulin glargine for one week or longer, after a single subcutaneous injection in type-2 diabetic ZDF rats.

\subsection{Materials and methods}

\subsubsection{Materials}

Insulin glargine (Lantus ${ }^{\circledR}$, Aventis Pharma, Parsippany, NJ) was purchased from Cardinal Health and insulin glargine particles were extracted using dialysis bags (Spectra/Por ${ }^{\mathbb{B}}$ Float-A-Lyzer ${ }^{\mathbb{B}}$, Biotech grade Regenerated Cellulose (RC), $5 \mathrm{~mL}, 3.5 \mathrm{~K}$ MWCO, Spectrum labs, Rancho Dominguez, CA). Labconco FreeZone ${ }^{\circledR} 12$ liter Console Freeze Dry System (Labconco, Kansas City, MO) was used for freeze drying. Recombinant human insulin (insulin) powder (26.9 IU/mg, $0.4 \%$ zinc) was purchased from Diosynth France S.A. (Usine St. Charles, France). The HPLC analysis of insulin was performed on a Shimadzu HPLC system (Shimadzu Scientific Instruments, Inc., Columbia, MD). The primary column was a 250 x $4.6 \mathrm{~mm}$ Altech Macrosphere RP 300 C18 $5 \mu \mathrm{m}$ column and the guard column was $7.5 \times 4.6 \mathrm{~mm}$ (Altech, Deerfield, IL), packed with same material as the primary column. HPLC grade Acetonitrile (ACN), trifloroacetic acid (TFA) and distilled water were used for preparing the mobile phase. The PLGA used in this study was Medisorb ${ }^{\circledR} 5050$ DL 1A (i.v. 0.09 dL/g) donated by Alkermers Inc. (Cambridge, MA). Triethyl citrate (TEC) and acetyltriethyl citrate (ATEC) donated by Morflex Inc., (Greensboro, NC) were used as plasticizers. male ZDF (fa/fa) rats (Charles River Laboratories, Inc., Wilmington, MA) weighing 300 to 400 grams were used for the in vivo studies. Blood glucose was measured using Bayer Ascensia ${ }^{\circledR}$ Breeze blood glucose meter (Bayer Corporation, Elkhart, IN). The concentration of glargine in the plasma or serum was determined using a Mercodia human insulin ELISA kit (Mercodia AB, Uppsala, Sweden). Blood glucose and glargine monitoring in the animals was stopped when the concentration of blood glucose went back to the normally high concentration.

\subsubsection{Methods}

4.2.2.1 Extraction of insulin glargine particles. The Spectra/Por ${ }^{\circledR}$ Float-A-Lyzer ${ }^{\circledR}$ (dialysis bags) were removed from the refrigerator and allowed to equilibrate to room temperature. The cap was removed and the sodium azide solution inside the bag was discarded using a pipette. The dialysis bags were then cleaned with distilled water and placed in a one liter beaker containing $900 \mathrm{~mL}$ of distilled water for one hour to remove 
any remaining sodium azide. The bags were removed from the beaker and again washed several times with distilled water. Insulin glargine (Lantus $\left.{ }^{\circledR}\right)$ solution $(5 \mathrm{~mL})$ was loaded into each bag using a pipette. The insulin glargine solution filled bags were kept in the beaker containing $900 \mathrm{~mL}$ of distilled water and the beaker with the dialysis bags was placed in an ice bath and stirred continuously with a magnetic stirrer for 24 hours. The distilled water in the beaker was completely replaced with fresh water after 2, 4, 8 and 12 hours. After 24 hours, the cap of the dialysis bag was carefully removed and the sample was recovered using a pipette. The recovered sample was placed in a pre-washed $20 \mathrm{~mL}$ scintillation vial, which was covered with aluminum foil. Holes were punctured in the aluminum foil using an 18 gauge needle. This aluminum foil covered scintillation vial was then placed in a freezer at $-80^{\circ} \mathrm{C}$ for 6 hours. The freeze dryer was then switched on and vacuum was allowed to come to the maximum concentration. The samples were removed from $-80^{\circ} \mathrm{C}$ and freeze dried for 12 hours. The dried samples were pooled together in a clean $20 \mathrm{~mL}$ scintillation vial and sealed with Parafilm, capped and stored at $-20^{\circ} \mathrm{C}$ until used for further studies.

4.2.2.2 High pressure liquid chromatography method of insulin glargine. The HPLC analysis of the freeze-dried insulin glargine was performed using a Shimadzu HPLC system (Shimadzu Scientific Instruments, Inc., Columbia, MD) consisting of an SCL-10A vp system controller, a LC-10 AD vp pump, a DGU-14A degasser, an SIL-10 Ad vp autoinjector with a cooling system, a CTO-10A vp column oven, an SPD-10AD vp photo-diode array (PDA) detector and a computer loaded with the Shimadzu Class-VP 7.2 software. The primary column was a 250 x $4.6 \mathrm{~mm}$ Altech Macrosphere RP $300 \mathrm{C} 18$ $5 \mu \mathrm{m}$ column and the guard column was $7.5 \times 4.6 \mathrm{~mm}$, packed with same material as the primary column.

A gradient elution method was used for analyzing the insulin. Mobile phase A was $0.15 \% \mathrm{v} / \mathrm{v}$ trifluoroacetic acid (TFA) in water and mobile phase B was $0.13 \% \mathrm{v} / \mathrm{v}$ TFA in $95 \% \mathrm{v} / \mathrm{v}$ acetonitrile in water. The mobile phases were filtered through a Whatmann $0.45 \mu \mathrm{m}$ nylon membrane (Whatmann International Ltd, Maidstone, England) and degassed in an ultrasonicator (Model FS60, Fisher Scientific, Fair Lawn, NJ) for 10 minutes before pumping it through the HPLC system.

The initial mobile phase composition of $70 \%$ solvent $\mathrm{A}$ and $30 \%$ solvent $\mathrm{B}$ was maintained at a flow rate of $1 \mathrm{~mL} / \mathrm{min}$ for 2 minutes. Between 2 and 16 minutes, the percentage of solvent B was increased linearly to $50 \%$. These conditions were maintained from 5 to 21 minutes. Between 22 and 25 minutes, the percentage of solvent B was decreased linearly to $30 \%$. The mobile phase was pumped at a flow rate of $1 \mathrm{~mL} / \mathrm{min}$. The injection volume of each sample, including the insulin glargine standards, insulin glargine controls, insulin glargine samples (unknown concentration) and blank samples $(0.01 \mathrm{~N} \mathrm{HCl})$ without insulin glargine), was $50 \mu \mathrm{L}$. The temperature of the HPLC column was maintained at $30^{\circ} \mathrm{C}$ by using a column oven. The primary detection wavelength of insulin was set to $285 \mathrm{~nm}$.

4.2.2.3 Preparation of standard, control and sample solutions. The standard and control solutions of insulin glargine were prepared from the commercially available 
Lantus ${ }^{\circledR}$ solution with a concentration of $3.63 \mathrm{mg} / \mathrm{mL}$ as the working stock solution. Standard solutions were prepared from the working stock solution by transferring 50, 75, 100,150 and $200 \mu \mathrm{L}$ of the working stock solution to five $2 \mathrm{~mL}$ volumetric flasks and making up the volume with $0.01 \mathrm{~N} \mathrm{HCl}(\mathrm{pH} 2.0)$.

The insulin glargine controls were also prepared from the working stock solution with a concentration of $100 \mu \mathrm{g} / \mathrm{mL}$. For sample preparations, approximately $5 \mathrm{mg}$ of freeze-dried insulin glargine particles were weighed on a calibrated Cahn C-31 microbalance (Cahn Instruments, Cerritos, CA) and transferred to a volumetric flask and dissolved in $0.01 \mathrm{~N} \mathrm{HCl}(\mathrm{pH} 2.0)$. The final volume was made with $0.01 \mathrm{~N} \mathrm{HCl}(\mathrm{pH} 2.0)$. The dissolved samples were immediately loaded on the HPLC tray.

\subsubsection{Process of chromatographic data. After each HPLC injection, the} software generated the chromatograph of the sample and integrated the peak area of insulin glargine. The peak area of the standard solutions was then plotted against the concentration of insulin glargine. A linear regression method was used to generate the equation from which the concentration of the unknown sample could be interpolated from the peak area. The concentration of the insulin glargine control was interpolated and compared to the theoretical value. The concentration of insulin glargine samples was determined using the standard curve.

4.2.2.5 Pharmacodynamic studies of insulin and insulin glargine in ZDF rats. All the experimental procedures for the animal studies were approved by the University of Tennessee Health Science Center's Animal Resources Advisory Committee. The ZDF rats were at least 16 weeks old (weighing 300 to $400 \mathrm{~g}$ ). Hyperglycemia ( $>400 \mathrm{mg} / \mathrm{dL}$ ) in the ZDF rats was confirmed by blood glucose measurements. The rats were group-housed (3 per cage) in the animal facility under a 12-hour light/dark cycle. They had access to food and water ad libitum throughout the studies. Before the animals were shipped from Charles River Laboratories, they were fed Purina diet \#5008 until they fully developed diabetes (12 weeks old). The animals were on regular laboratory rodent food in our facility. The rats were acclimated to the environment for at least three days before they were used in the studies.

Insulin (RHI), insulin glargine and the commercially available formulation of insulin glargine, Lantus ${ }^{\circledR}$ were administered to the ZDF rats (type 2 diabetes) by a subcutaneous injection. An appropriate amount of insulin or insulin glargine powder was suspended in sterile normal saline to obtain a suspension with a concentration of 40 IU/mL (Table 4-1). Lantus ${ }^{\circledR}$ was used as a control and was administered to the animals as is $(100 \mathrm{IU} / \mathrm{mL})$.

Blood glucose concentrations in each animal were determined following a subcutaneous injection of each form of insulin at a dose of $10 \mathrm{IU} / \mathrm{kg}$ as follows: On each day of the study, the ZDF rats were transferred to the procedure room. The rats were randomly divided into groups, with 4 to 6 animals per group. Each group, except the control group, received one dose of a form of insulin. The control group did not receive any insulin injection. Before injection, each rat was placed in an induction chamber and 
Table 4-1 Composition of gel formulations used for in vivo studies

\begin{tabular}{|c|c|c|c|c|c|}
\hline $\begin{array}{c}\text { Formulation } \\
\#\end{array}$ & $\begin{array}{c}\text { Concentration } \\
\text { of polymer } \\
*(\% \text { w/w })\end{array}$ & $\begin{array}{c}\text { Concentration } \\
\text { of ATEC } \\
(\% \mathrm{w} / \mathrm{w})\end{array}$ & $\begin{array}{c}\text { Concentration } \\
\text { of TEC } \\
(\% \mathrm{w} / \mathrm{w})\end{array}$ & $\begin{array}{l}\text { Insulin } \\
\text { glargine } \\
(\% \text { w/w })\end{array}$ & $\begin{array}{c}\text { Zinc } \\
\text { Sulfate } \\
(\% \text { w/w })\end{array}$ \\
\hline OA-G-1 & - & - & - & 4.0 & - \\
\hline OA-G-2 & - & 72 & 24 & 4.0 & - \\
\hline OA-G-3 & 5.0 & 69.75 & 23.25 & 2.0 & - \\
\hline OA-G-4 & 5.0 & 68.25 & 22.75 & 4.0 & - \\
\hline OA-G-5 & 10.0 & 64.5 & 21.5 & 4.0 & - \\
\hline OA-G-6 & 5.0 & 67.88 & 22.62 & 4.0 & 0.50 \\
\hline OA-G-7 & 5.0 & 67.9 & 22.9 & 4.0 & 0.25 \\
\hline OA-G-8 & 5.0 & 68 & 22.9 & 4.0 & 0.10 \\
\hline
\end{tabular}

OA-G-1 was prepared in phosphate buffer saline (PBS)

* Inherent viscosity of the polymer (dL/g) - 0.09 
anesthetized by isoflurane (Halocarbon Product Corporation, River Edge, NJ) using a SurgiVet/Anesco ventilator (Waukesha, WI). The body weight of the animal was recorded to determine the volume of the insulin suspensions to be injected. Blood glucose was measured using Bayer Ascensia Breeze blood glucose meter (Bayer Corporation, Elkhart, IN). The predetermined dose of insulin (which was determined based on the animal body weight) was injected subcutaneously at the dorsal neck of the rat using a $1 / 2$ $\mathrm{mL}$ Becton Dickinson Lo-Dose ${ }^{\mathrm{TM}} \mathrm{U}-100$ insulin syringe with a permanently attached 28 gauge $\mathrm{x} 1 / 2$ inch needle (Franklin Lakes, NJ). After injection, the rats were returned to the cages and allowed to recover from anesthesia. At pre-determined time intervals, the rats were anesthetized by the aforementioned method and their blood glucose was measured. A plot of blood glucose concentrations versus time was generated from the data to describe the glucose lowering effect of insulin or insulin glargine, from which information such as time of peak action and duration of action could be obtained.

4.2.2.6 Fabrication of biodegradable gels. The gel formulations (Table 4-1) were prepared in two steps: dissolution of polymer in the plasticizer(s) to obtain a blank gel and loading of insulin glargine into the blank gel.

The polymer was allowed to come to room temperature in a vacuum desiccator after it was taken out from the freezer. Then an appropriate amount of polymer particles was accurately weighed into a dry, clean glass scintillation vial using a Mettler AE100 analytical balance (Mettler-Toledo, Inc., Columbus, $\mathrm{OH}$ ). The desired amount of plasticizer(s) was weighed into the scintillation vial containing the polymer. A plastic screw cap with aluminum liner was used to seal the vial. The vial was then vortexed on a vortex mixer to disperse the polymer particles in the plasticizer(s). The vial was placed in a Lab-Line orbital shaker (Model 3527, Lab-line, Melrose Park, IL), which was maintained at $37^{\circ} \mathrm{C}$, and shaken at $150 \mathrm{rpm}$. It took 2 to 3 days for the polymer to completely dissolve in the plasticizer. The resulting blank gel was stored in vacuum desiccators at room temperature until further use (typically 1 to 2 days). One day before the insulin glargine-loaded gel was required, a desired amount of blank gel was transferred to a new glass scintillation vial and an appropriate amount (approximately 80 $\mathrm{mg}$ ) of insulin glargine particles was accurately weighed and transferred into the blank gel vial. A stainless steel spatula was used to disperse the insulin glargine particles uniformly into the blank gel. Any visible agglomerates of insulin glargine were broken using a spatula and the insulin glargine-loaded gel was ready to be used for the in vivo studies.

\subsubsection{In vivo studies of insulin glargine-loaded gel formulations in ZDF rats.} For the in vivo studies, blood glucose concentrations of the diabetic rats were measured before and after injection of insulin glargine-loaded gels. The concentration of insulin glargine of some of the formulations in plasma or serum was also determined and the data were plotted against time to characterize the release of insulin glargine from the insulin glargine-loaded gel formulations. For gel formulations consisting of insulin glargine and zinc sulfate, an appropriate quantity of zinc sulfate powder $(<\# 400$ mesh or $37.5 \mu \mathrm{m})$ was accurately weighed and added to the insulin glargine-loaded gel. 
Typically, a total of $2 \mathrm{~g}$ of gel (including insulin glargine and zinc sulfate) was prepared for each gel formulation. On the day of injection, the ZDF rats were transferred from the housing room to the procedure room. The animals were anesthetized by isoflurane. The dorsal neck of each rat (except the animals in the control group that did not receive any injection) was shaved to expose the injection site. Body weight of the rat was recorded. Blood glucose was measured prior to any injection using a glucose meter as described before. The insulin glargine-loaded gel was withdrawn into a barrel of a 1 $\mathrm{mL}$ syringe, without any needle. Then a 23 gauge needle ( 1 inch in length) was attached to the syringe, and the volume of the gel in the syringe was adjusted to the desired volume, depending on the dose of insulin for each animal. The shaved area on the dorsal neck of the animal was disinfected with isopropyl swab and a subcutaneous injection of the insulin glargine-loaded gel was made. The needle was inserted under the skin in such a way that the gel would be injected between the skin and the underlying muscle tissue. The actual procedure of injecting the gel was as follows: At the injection site, the skin was pinched and lifted by the finger and thumb. The needle was inserted under the skin between the finger and thumb. Approximately three-quarters of an inch of needle was pierced into the skin to make sure that the injected gel would not ooze out after the needle was removed from the injection site. After injection of the gels, at pre-determined time intervals, the rats were anesthetized and blood glucose concentrations were measured. Blood samples ( 300 to $400 \mu \mathrm{L}$ ) were taken from the tail vein and processed to collect plasma or serum. These plasma or serum samples were stored at $-20^{\circ} \mathrm{C}$ until analysis.

4.2.2.8 Statistical analysis. Area under the effect curve (AUEC) which is defined as the area below baseline is representative of the effect of the formulation on lowering of blood glucose with greater AUEC implying greater effect. One way ANOVA within Excel 2007 was utilized to compare AUEC between treatment $(\mathrm{N}=4)$ and control $(\mathrm{N}=$ 4) at $95 \%$ confidence level.

\subsection{Results and discussion}

\subsubsection{Purity of insulin glargine particles}

Figure 4-1 shows the chromatogram of placebo Lantus ${ }^{\circledR}$ formulation (without any insulin glargine). The placebo was made in house and each milliliter of the formulation contained $2.7 \mathrm{mg}$ of m-cresol, $30 \mu \mathrm{g}$ of zinc, (from zinc chloride), $20 \mathrm{mg}$ of glycerol $85 \%$ $\mathrm{w} / \mathrm{w}$ and water for injection. This formulation was similar to Lantus ${ }^{\circledR}$ formulation without insulin glargine. It is evident from the Figure 4-1 that the retention time of the peak of the placebo formulation is approximately 6 minutes.

Figure 4-2 shows the chromatogram of Lantus ${ }^{\circledR}$ formulation diluted in $0.01 \mathrm{~N}$ $\mathrm{HCl}$. The chromatogram shows two peaks, one at 6 minutes, which corresponds to the placebo peak and another peak at 11 minutes. The peak at 11 minutes is the peak of insulin glargine or Lantus ${ }^{\mathbb{B}}$. The diluted Lantus ${ }^{\circledR}$ formulations were injected at various concentrations and the areas under the peaks at 11 minutes were used to prepare the standard plot. 


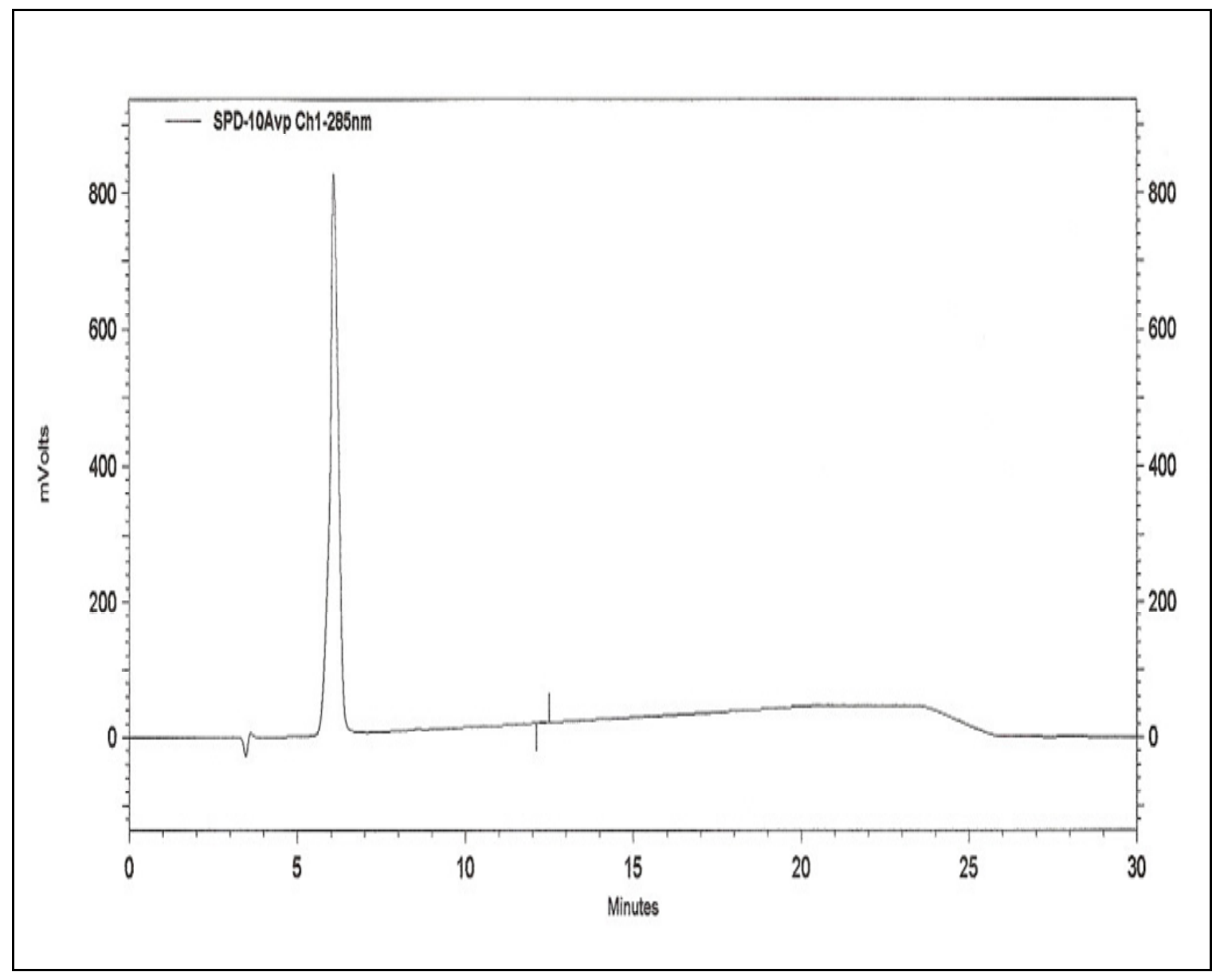

Figure 4-1 HPLC chromatogram of placebo of Lantus ${ }^{\circledR}$ formulation 


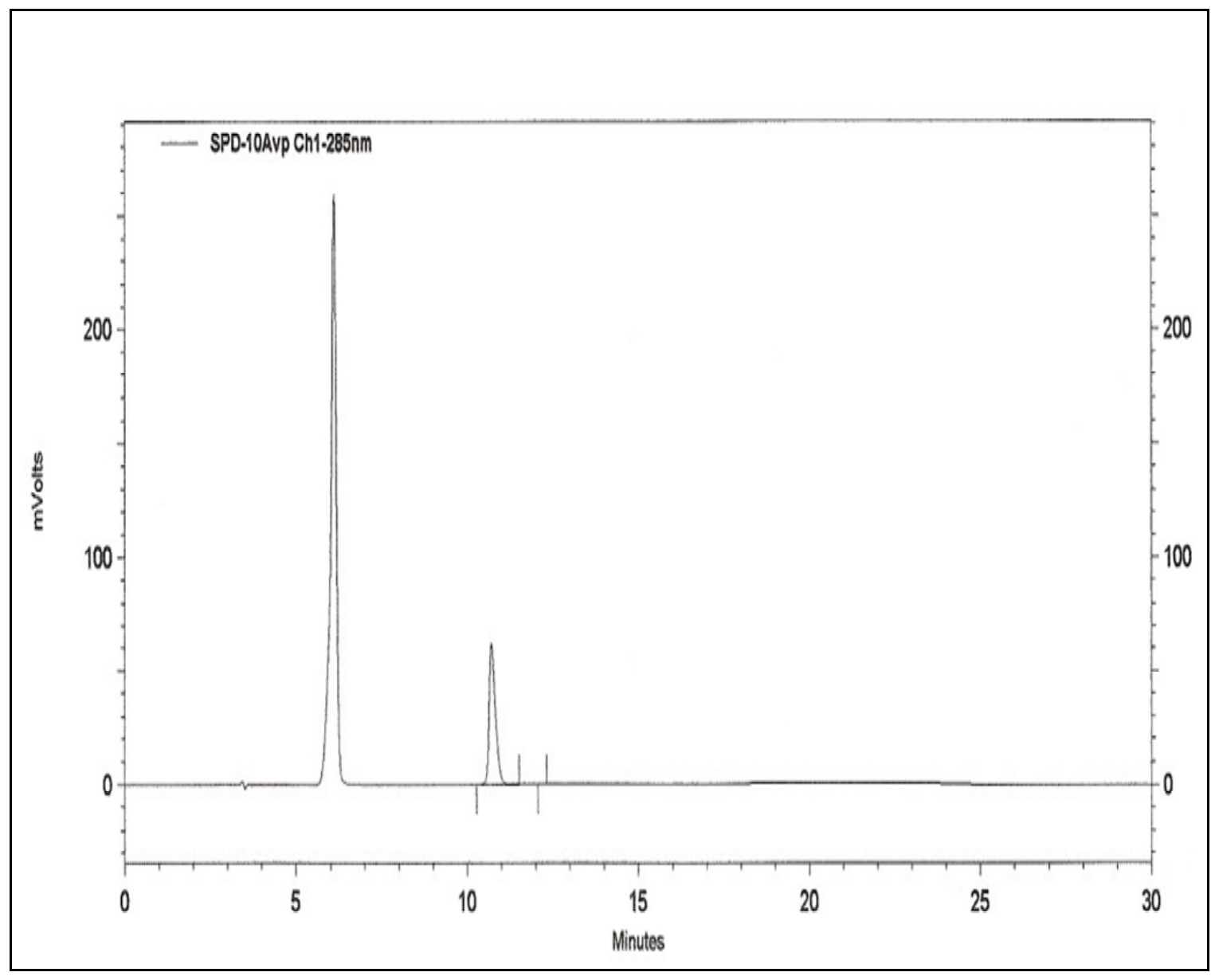

Figure 4-2 HPLC chromatogram of Lantus ${ }^{\circledR}$ formulation 
Figure 4-3 shows the chromatogram of purified (dialyzed) and freeze-dried particles of insulin glargine dissolved in $0.01 \mathrm{~N} \mathrm{HCl}$. The chromatogram shows a single peak at 11 minutes. It is evident from the Figure 4-3 that the placebo peak at 6 minutes is missing, thus indicating that the excipients in the Lantus ${ }^{\circledR}$ formulation were removed using the dialysis bags and pure insulin glargine particles were obtained after freeze drying. The calculated purity of these insulin glargine particles was found to be $94.6 \%$.

\subsubsection{Pharmacodynamic studies of insulin formulations in ZDF rats}

Insulin and insulin glargine particles were suspended in sterile normal saline $(0.9 \% \mathrm{NaCl})$ and administered subcutaneously to ZDF rats to evaluate the biological effect of different forms of insulin on the blood glucose concentrations of the rats. Figure 4-4 shows the blood glucose concentrations of these pharmacodynamic studies. It is evident from Figure 4-4 that the blood glucose concentrations decreased from approximately $452 \pm 27.38 \mathrm{mg} / \mathrm{dL}$ to $92.5 \pm 4.9 \mathrm{mg} / \mathrm{dL}$ within 3 hours after a subcutaneous injection of $10 \mathrm{IU} / \mathrm{kg}$ dose of RHI in the ZDF rats. Normal blood glucose concentrations (between $80-200 \mathrm{mg} / \mathrm{dL}$ ) were maintained for approximately an hour before reaching the normally high blood glucose concentrations within 6 hours. Lantus ${ }^{\circledR}$ was subcutaneously injected at dose of $10 \mathrm{IU} / \mathrm{kg}$ (Figure 4-4). The blood glucose concentrations after Lantus ${ }^{\circledR}$ injection decreased from approximately $441 \pm 17.02 \mathrm{mg} / \mathrm{dL}$ to $229 \pm 45 \mathrm{mg} / \mathrm{dL}$ within 4 hours post injection. The blood glucose concentrations after subcutaneous injection of purified glargine particles suspended in saline decreased from approximately $481 \pm 16.68 \mathrm{mg} / \mathrm{dL}$ to $136.23 \pm 14.56 \mathrm{mg} / \mathrm{dL}$ within 2 hours (Figure 4-4).

Normal blood glucose concentrations (between 80 and $200 \mathrm{mg} / \mathrm{dL}$ ) were maintained for approximately 6 hours before reaching the normally high blood glucose concentrations $(350 \mathrm{mg} / \mathrm{dL})$ within 12 hours. This pharmacodynamic effect of lowering of blood glucose concentrations shows that the insulin glargine particles (purified from the commercially available Lantus ${ }^{\circledR}$ formulation) and suspended in normal saline were bioactive. There was a significant difference between AUEC after subcutaneous injection of insulin glargine particles and control at 95\% confidence level $(\mathrm{P}=0.003)$ indicating that insulin glargine particles causes significantly greater reduction in blood glucose when compared to control. Moreover, no significant difference was observed between AUEC after subcutaneous injection of insulin glargine particles and Lantus ${ }^{\circledR}(\mathrm{P}>0.05)$ at $95 \%$ confidence level indicating that the Lantus ${ }^{\mathbb{R}}$ is no better than insulin glargine particles in reduction of blood glucose.

\subsubsection{In vivo studies of gel formulations loaded with insulin glargine particles}

Purified and freeze-dried particles of insulin glargine were suspended in phosphate buffer saline (PBS) at 4\% loading, and $0.1 \mathrm{~mL}$ of this suspension was injected subcutaneously in the ZDF rats. Figure 4-5 shows the changes in blood glucose concentrations and body weights of ZDF rats after the injection. It is evident from the figure that blood glucose concentrations decreased rapidly below $100 \mathrm{mg} / \mathrm{dL}$ within 6 hours after injection and remained at that concentration until day 3 before rapidly climbing climbing to above $300 \mathrm{mg} / \mathrm{dL}$ on day 5 . 


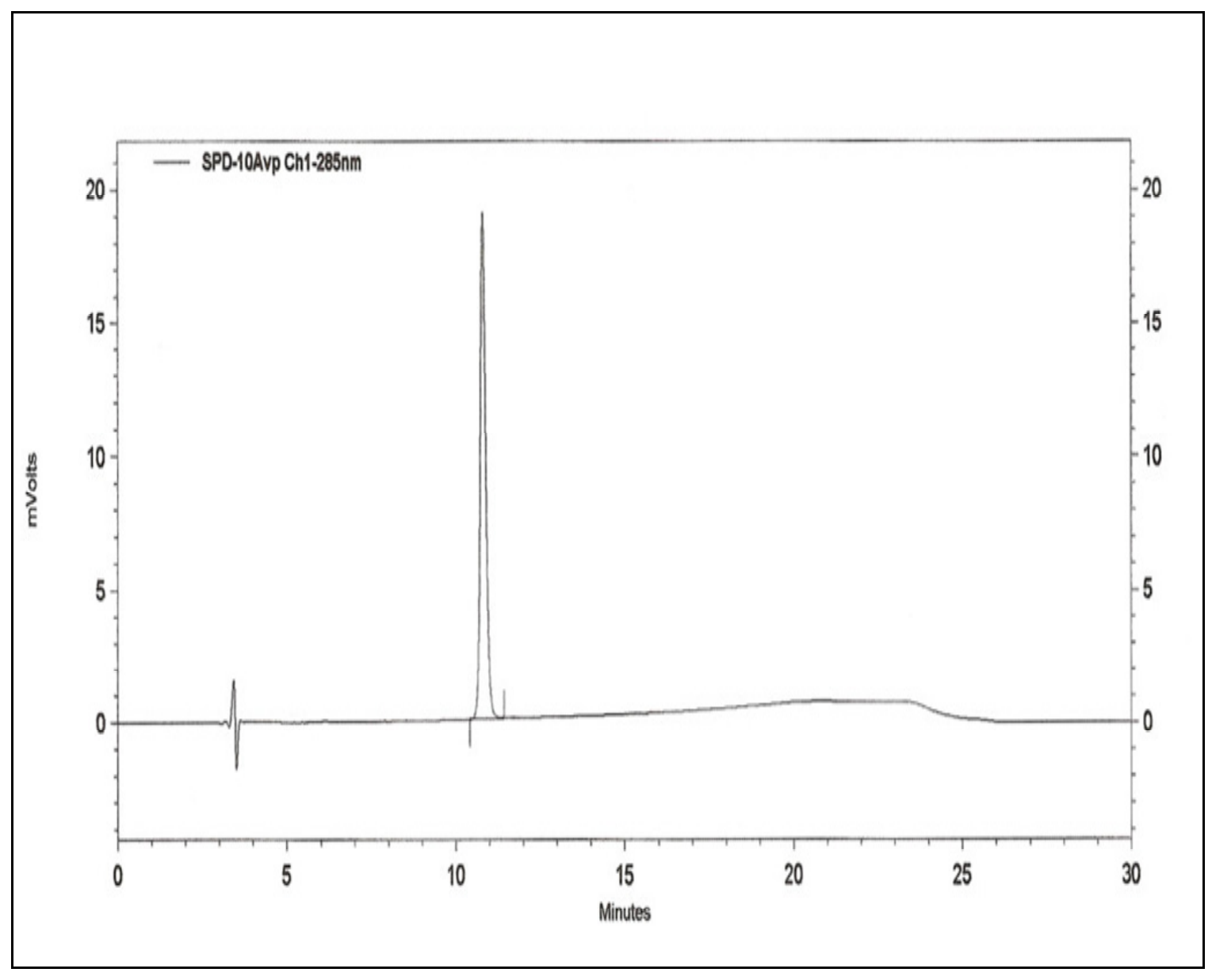

Figure 4-3 HPLC chromatogram of insulin glargine particles dissolved in $\mathrm{HCl}$ 


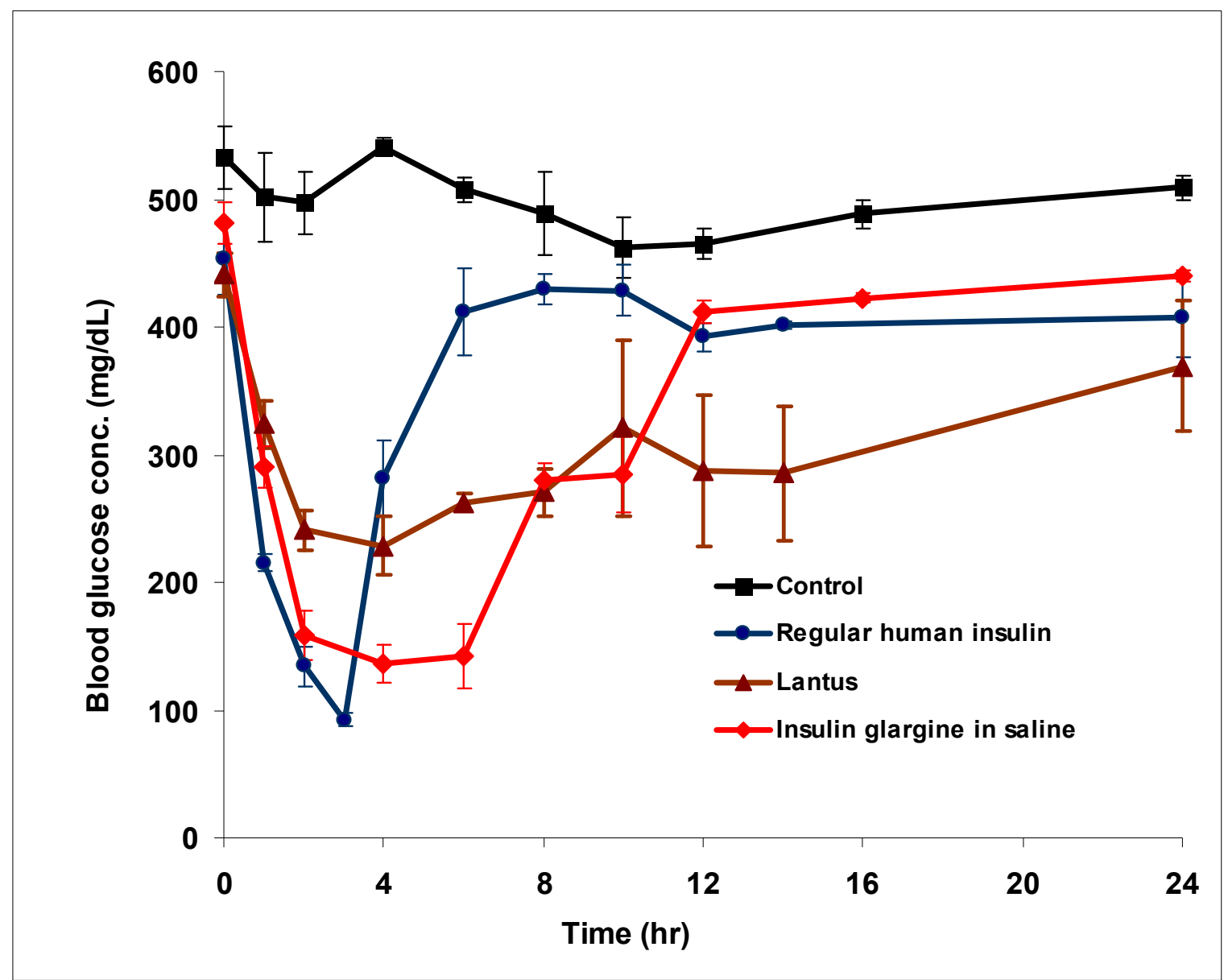

Figure 4-4 Blood glucose profiles in ZDF rats after subcutaneous injections of insulin (RHI), Lantus ${ }^{\circledR}$ and insulin glargine particles suspended in saline (dose-10 U/Kg) (Mean \pm SEM, $n=5$ ) 


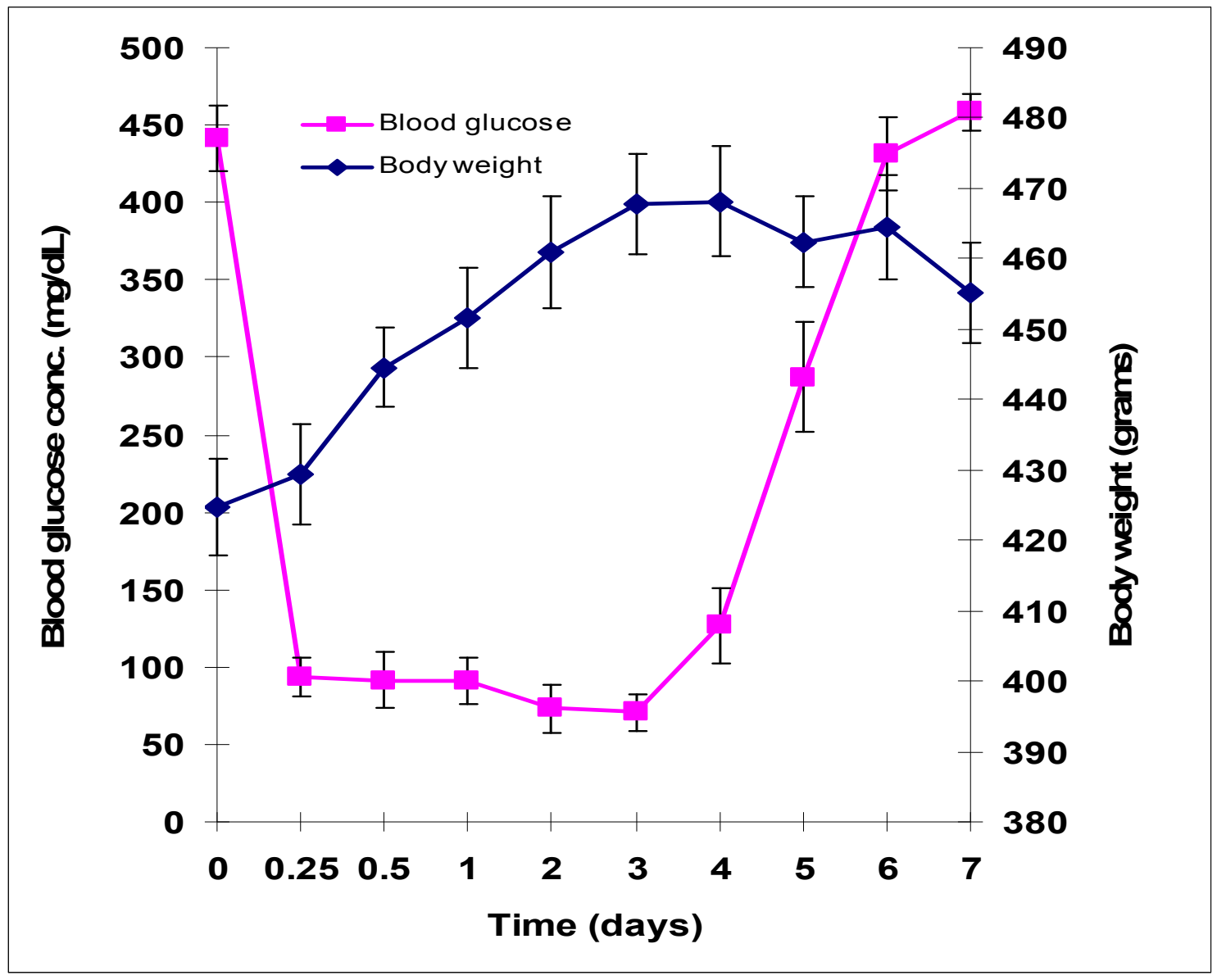

Figure 4-5 Changes in blood glucose concentrations and body weights of ZDF rats after subcutaneous injections of $4 \%$ suspension of insulin glargine particles in PBS $($ Mean \pm SEM, $\mathrm{n}=5$ ) 
The rapid decline in blood glucose concentrations is not desirable. Moreover, the rats were nearly hypoglycemic for three days. Hence, there was a need to further slow down the release of insulin glargine, which produced a slower and extended effect in the animals. However, from Figure 4-5, it is clear that as the blood glucose concentrations decreased, the body weights of the rats increased rapidly. Moreover, the body weights of the animals started to decline as the blood glucose concentrations climbed back to the normally high concentrations above $350 \mathrm{mg} / \mathrm{dL}$. This showed that the glargine particles not only produced a lowering in blood glucose concentrations, but also affected the body weights, thus proving that the purified and freeze-dried particles of insulin glargine were bioactive.

A similar effect in the change in blood glucose concentrations and body weights of ZDF rats was observed after subcutaneous injection of $0.1 \mathrm{~mL}$ of $4 \%$ suspension of insulin glargine particles in 3:1 blend of ATEC: TEC plasticizers (Figure 4-6). Hence, it is clear from this study that loading insulin glargine particles in a combination of plasticizers alone does not provide extension of biological effect of insulin glargine. Figure 4-7 depicts change in blood glucose concentrations and body weights of ZDF rats after a subcutaneous injection of PLGA gel formulation (containing 5\% PLGA) loaded with insulin glargine particles. The drug loading in the gel formulation (Formulation-OAG-3) as shown in Table 4-1 was $2 \% \mathrm{w} / \mathrm{w}$. The drug loading in the gel formulation (Formulation-OA-G-3) as shown in Table $4-1$ was $2 \% \mathrm{w} / \mathrm{w}$. The blood glucose concentrations reduced from $425 \mathrm{mg} / \mathrm{dL}$ to approximately $100 \mathrm{mg} / \mathrm{dL}$ within 24 hours after the injection and remained between 100 and $200 \mathrm{mg} / \mathrm{dL}$ for 4 days before steadily climbing back up to above $300 \mathrm{mg} / \mathrm{dL}$ on the day 9 . This showed that addition of $5 \%$ PLGA to the plasticizer combination of ATEC:TEC (3:1) not only slowed down the in vivo effect of insulin, but also extended the duration of action of the drug for approximately 6 days before climbing back up to the normally high concentrations of above $350 \mathrm{mg} / \mathrm{dL}$.

Figure 4-8 shows changes in blood glucose concentrations and body weights of ZDF rats after subcutaneous injection of gel formulation prepared with 5\% PLGA and loaded with $4 \% \mathrm{w} / \mathrm{w}$ of insulin glargine particles (Formulation OA-G-4) (Table 4-1). It is evident from the figure that the effect of this gel formulation (Formulation OA-G-4) was similar to Formulation OA-G-3 with $2 \%$ drug loading. The blood glucose concentrations dropped from $445 \mathrm{mg} / \mathrm{dL}$ to about $100 \mathrm{mg} / \mathrm{dL}$ in 24 hours, and remained below 150 $\mathrm{mg} / \mathrm{dL}$ for 9 days, before rising steadily above $300 \mathrm{mg} / \mathrm{dL}$ on day 13 . Hence, doubling the drug loading from $2 \%$ (Formulation OA-G-3) to 4\% (Formulation OA-G-4) extended the maintenance of normal blood glucose concentrations from 6 to 9 days, respectively. Moreover, the effect of insulin glargine was also seen on the body weights, which steadily increased as the blood glucose concentrations dropped. The body weights remained high and dropped slightly when the blood glucose concentrations began to rise steadily.

To explore the possibility of slower and longer duration of effect of insulin glargine in the ZDF rats, the polymer concentration in the gel formulation was increased 


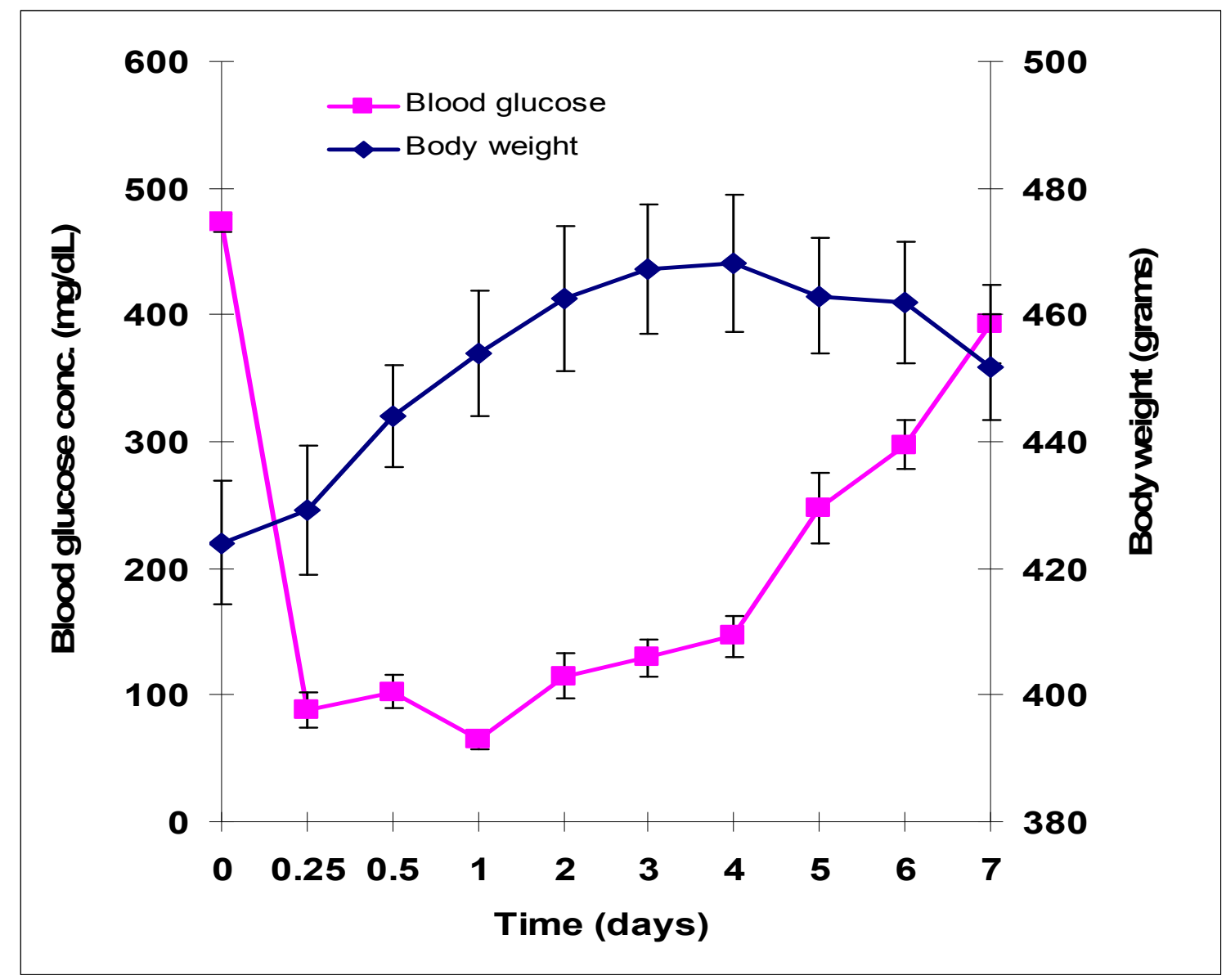

Figure 4-6 Changes in blood glucose concentrations and body weights of ZDF rats after subcutaneous injections of $4 \%$ suspension of insulin glargine particles in blends of ATEC:TEC (3:1) (Mean \pm SEM, $\mathrm{n}=5)$ 


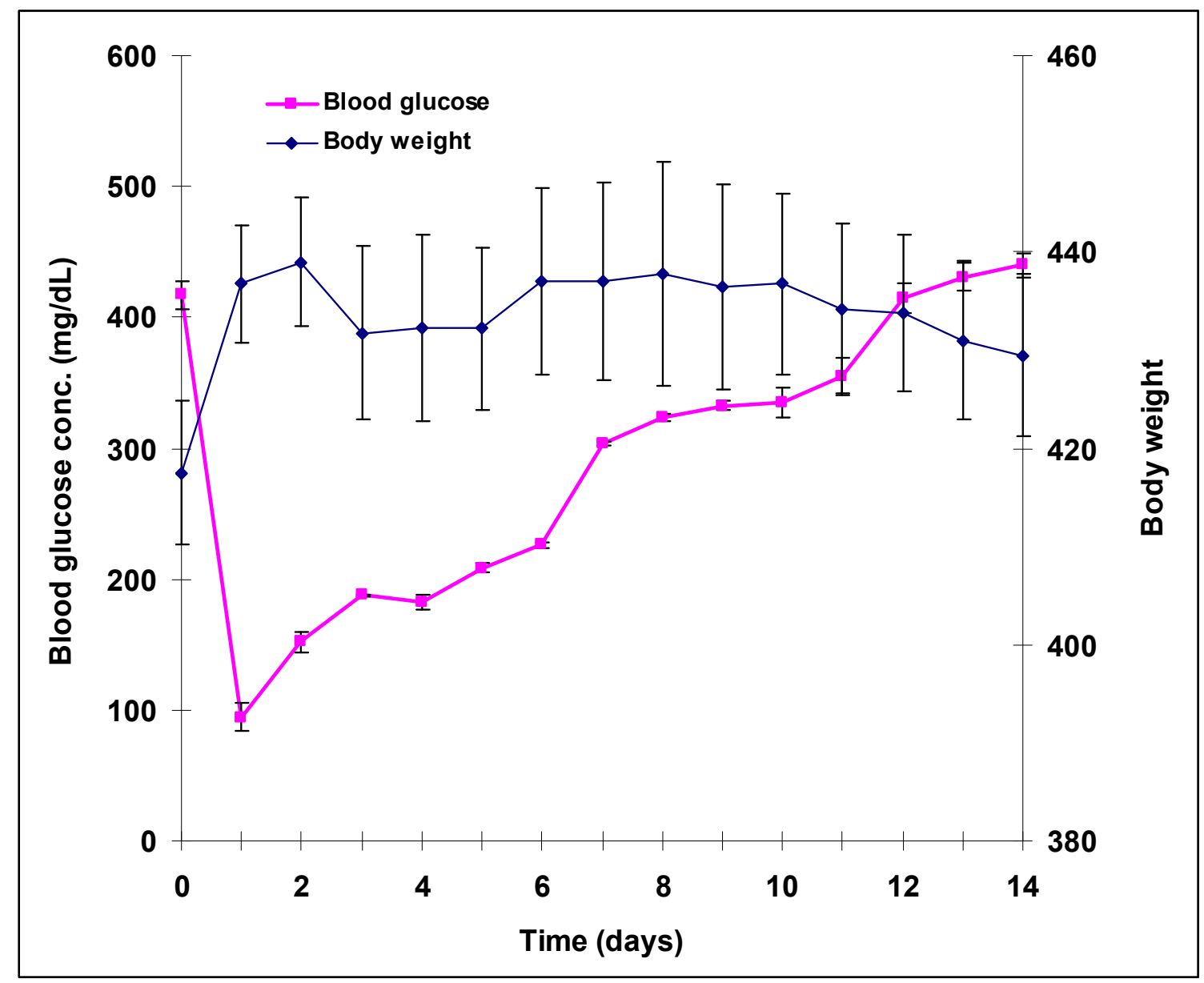

Figure 4-7 Changes in blood glucose concentrations and body weights of ZDF rats after a subcutaneous injection of gel (5\% PLGA) formulation loaded with $2 \%$ insulin glargine particles (Mean \pm SEM, $n=5$ ) 


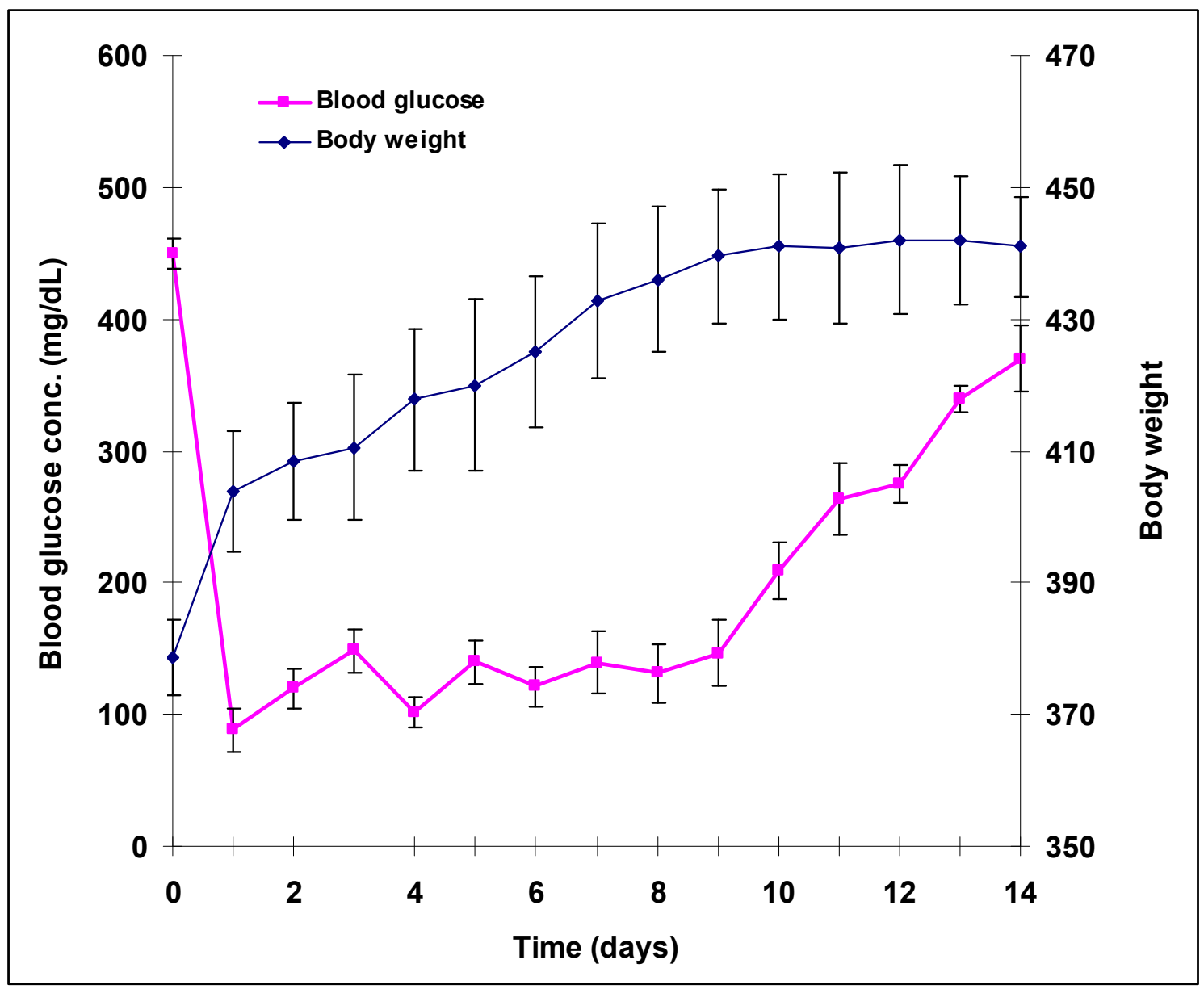

Figure 4-8 Changes in blood glucose concentrations and body weights of ZDF rats after a subcutaneous injection of gel (5\% PLGA) formulation loaded with $4 \%$ insulin glargine particles (Mean $\pm \mathrm{SEM}, \mathrm{n}=5$ ) 
from 5 to $10 \% \mathrm{w} / \mathrm{w}$. Figure 4-9 shows changes in blood glucose concentrations and body weights of ZDF rats after subcutaneous injection of PLGA gel (prepared with 10\% PLGA) formulation loaded with 4\% insulin glargine particles (Formulation OA-G-5) (Table 4-1).

The changes in blood glucose concentrations and body weights of ZDF rats were similar to Formulation OA-G-4 containing 5\% PLGA gel (Figure 4-8). Hence, increasing the PLGA from $5 \%$ to $10 \%$ did not increase the in vivo duration of activity of insulin in the ZDF rats. Figure 4-10 depicts the comparison of blood glucose concentrations in ZDF rats after subcutaneous injections of different formulations of insulin glargine with $4 \%$ loading with (Formulation OA-G-4) or without PLGA (Formulation OA-G-1 \& Formulation OA-G-2). There was a significant difference between AUEC after subcutaneous injection of formulation of insulin glargine with 4\% loading with PLGA (Formulation OA-G-4) and control at 95\% confidence level $(\mathrm{P}=0.00001)$ indicating that insulin glargine with 4\% loading with PLGA causes significantly greater reduction in blood glucose when compared to control. Furthermore, There was a significant difference between AUEC after subcutaneous injection of formulation of insulin glargine with 4\% loading with PLGA (Formulation OA-G-4) and formulation of insulin glargine with 4\% loading without PLGA (Formulation OA-G-1) at 95\% confidence level $(\mathrm{P}=0.0004)$ indicating that insulin glargine with $4 \%$ loading with PLGA causes significantly greater reduction in blood glucose concentrations, when compared to insulin glargine with $4 \%$ loading without PLGA.

Moreover, no significant difference was observed between AUEC after subcutaneous injection formulation of insulin glargine with $4 \%$ loading without PLGA i.e insulin glargine in PBS (Formulation OA-G-1) and insulin glargine in plasticizers (Formulation OA-G-2) ( $>>0.05)$ at 95\% confidence level indicating that the insulin glargine in plasticizers (Formulation OA-G-2) is no better than insulin glargine in PBS (Formulation OA-G-1), in reduction of the blood glucose concentrations. It is clear from the figure that addition of 5\% w/w PLGA not only slows down the lowering of blood glucose concentrations, but also increased the duration of action from 3 to 9 days. This extension in the blood glucose lowering effect of insulin glargine particles is due to presence of PLGA. This effect may be due to formation of a depot of insulin glargine particles encapsulated in the PLGA matrix. This PLGA matrix released the insulin slowly as it underwent erosion with time.

To study the concentration concentrations of glargine and its effect on blood glucose concentrations, Formulations OA-G-1 and OA-G-4 were injected into two different groups of $7 \mathrm{ZDF}$ rats. The blood glucose concentrations and concentrations of glargine in serum were determined. Figure 4-11 shows the concentrations of glargine in serum and blood glucose concentrations after a subcutaneous injection of Formulation OA-G-1. The concentration of glargine in serum of the ZDF rats increased very rapidly to $3563 \pm 1228 \mathrm{mIU} / \mathrm{L}$ within 6 hours after injection. The concentration of glargine remained above $1500 \mathrm{mIU} / \mathrm{L}$ until day 3 and then slowly declined below $100 \mathrm{mIU} / \mathrm{L}$ within 6 days after injection. The blood glucose concentrations dropped to a low concentration of $93 \pm 31 \mathrm{mg} / \mathrm{dL}$ within 6 hours after injection thus corresponding to the 


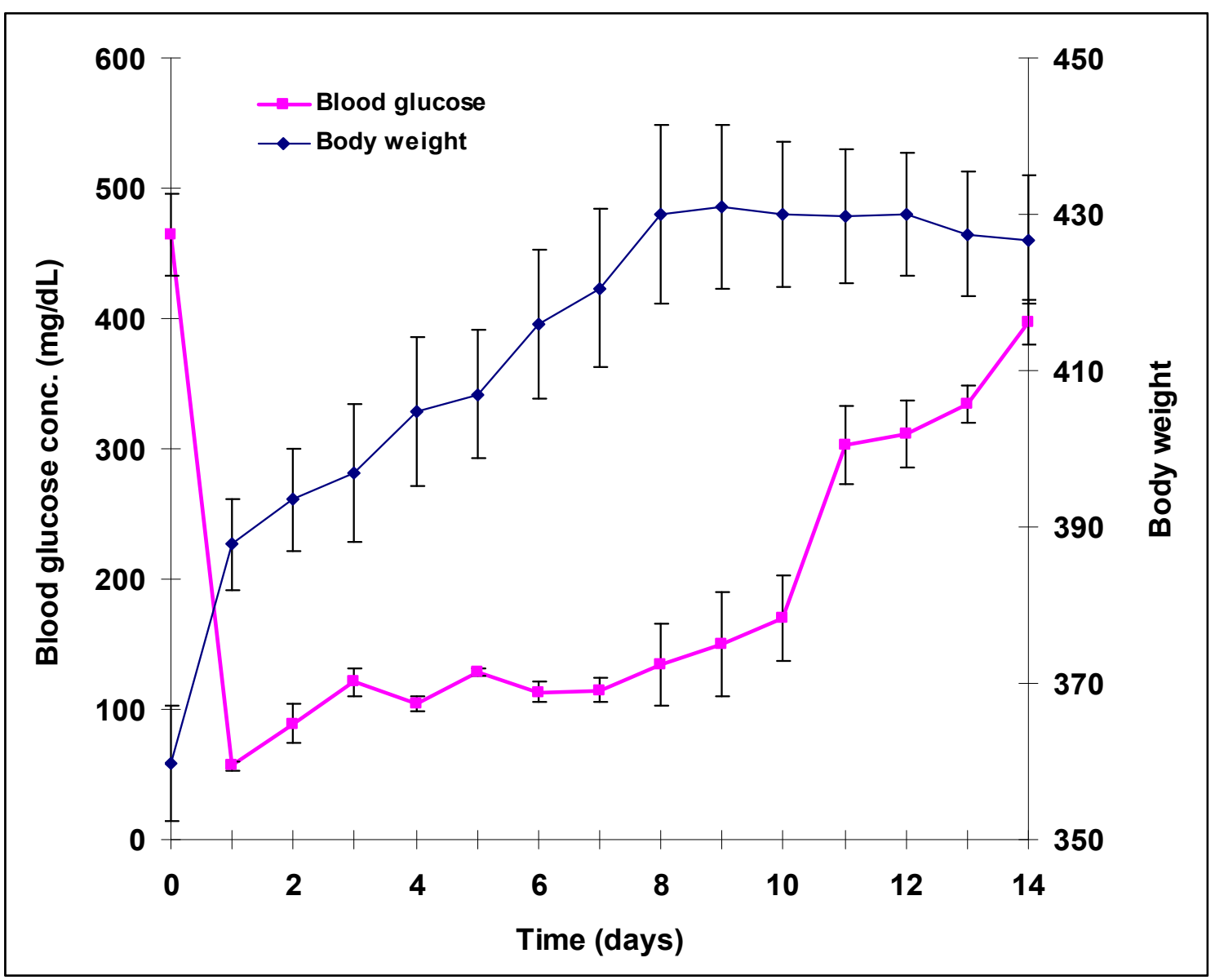

Figure 4-9 Changes in blood glucose concentrations and body weights of ZDF rats after subcutaneous injections of gel (10\%) formulations formulation loaded with $4 \%$ insulin glargine particles (Mean \pm SEM, $\mathrm{n}=5$ ) 


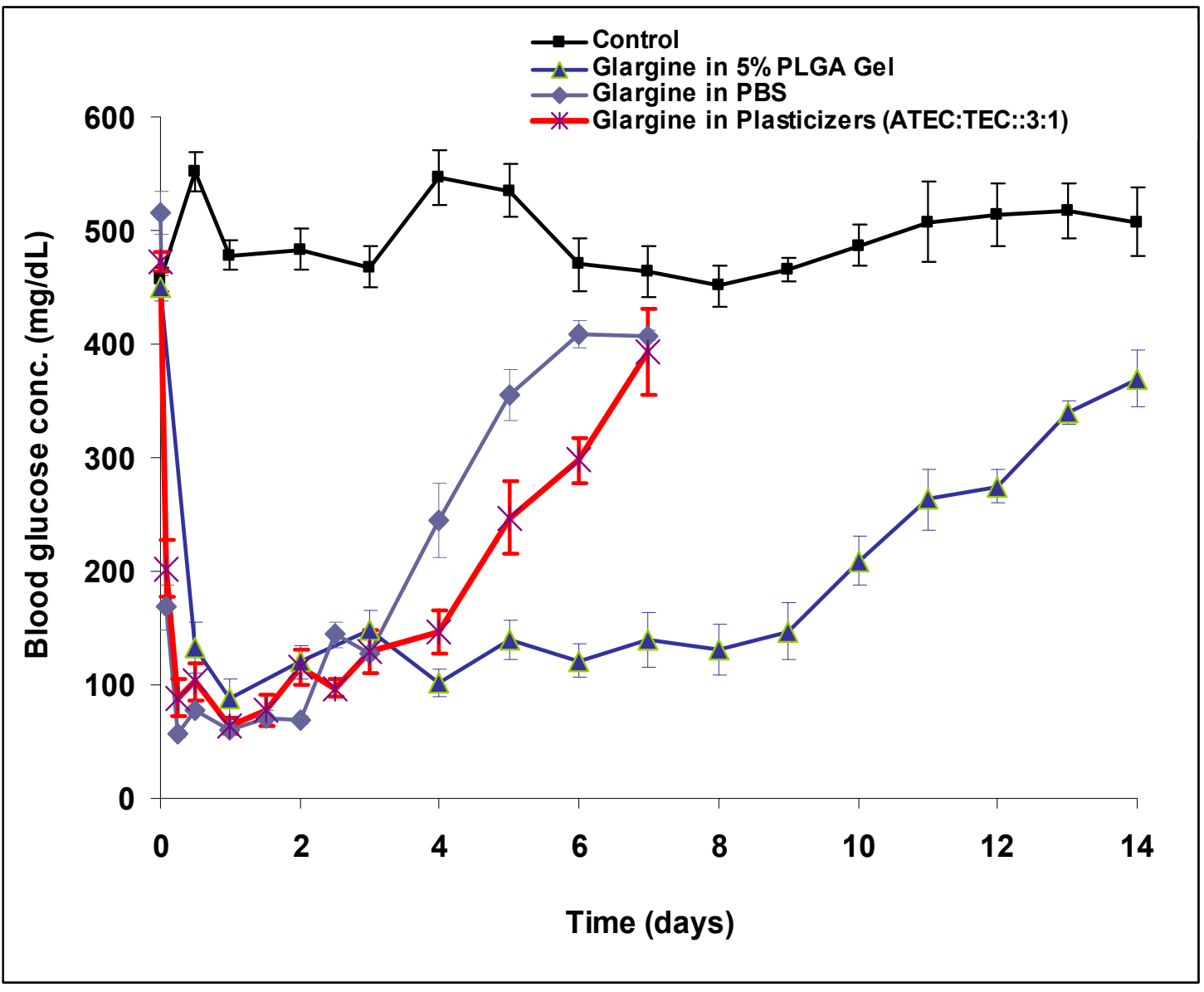

Figure 4-10 Comparison of blood glucose concentrations in ZDF rats after subcutaneous injections of insulin glargine (4\% loading) in different formulations (Mean $\pm \mathrm{SEM}, \mathrm{n}=5$ ) 


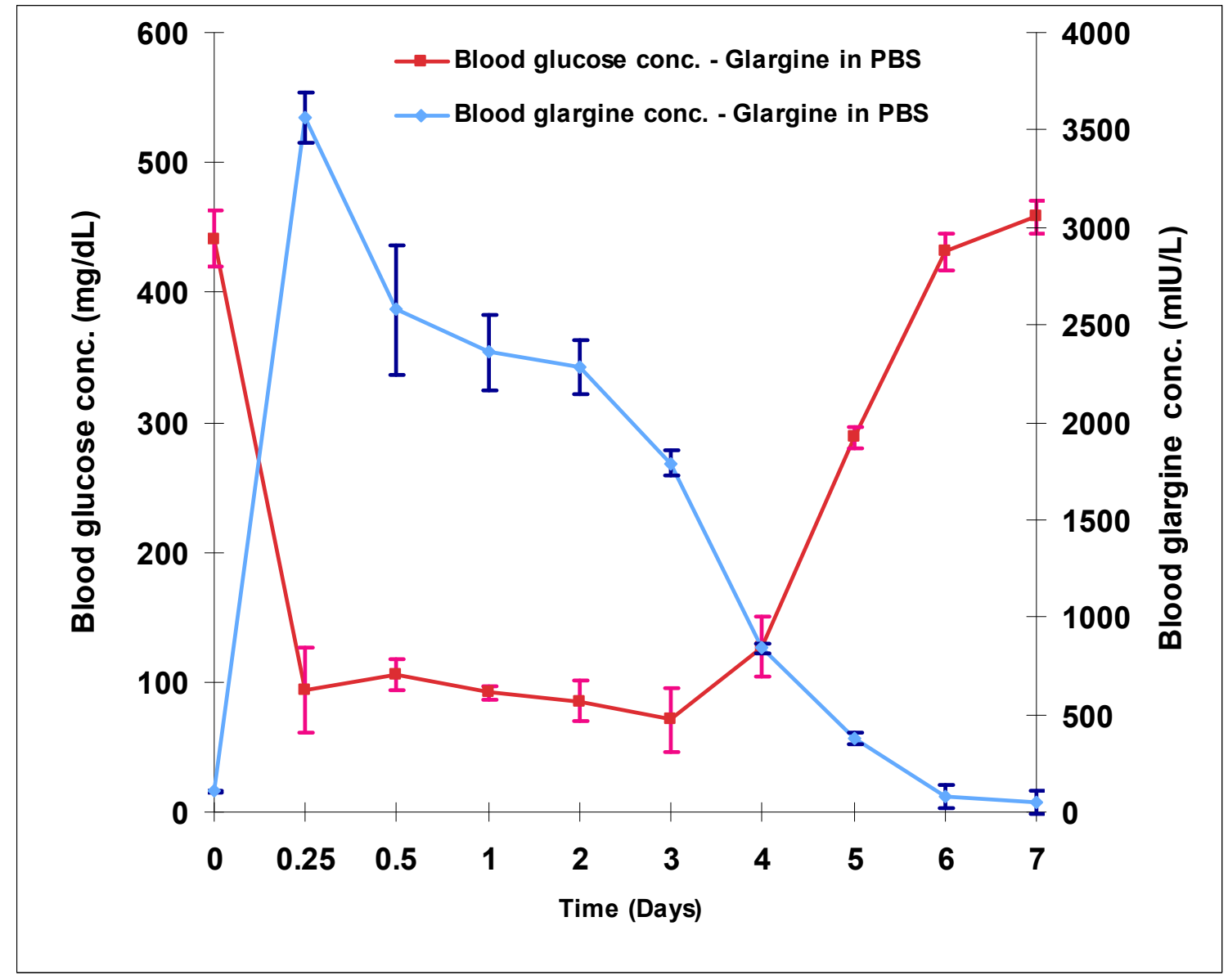

Figure 4-11 Blood glucose concentrations and concentrations of insulin glargine in serum of ZDF rats after a single subcutaneous injection of Formulation OA-G-1 (Mean \pm $\mathrm{SEM}, \mathrm{n}=7$ ) 
burst release of glargine from the formulation. The blood concentrations remained below $100 \mathrm{mg} / \mathrm{dL}$ for up to 4 days before it gradually went back up. The blood glucose concentrations went back up to more than $400 \mathrm{mg} / \mathrm{dL}$ on day 6 due to the decline in glargine serum concentrations.

Figure 4-12 shows the concentration of glargine in serum of the ZDF rats and blood glucose concentrations after a subcutaneous injection of gel Formulation OA-G-4. The concentration of glargine in serum of the ZDF rats increased to approximately 750 $\mathrm{mIU} / \mathrm{L}$ within 12 hours after injection, and then declined to approximately $500 \mathrm{mIU} / \mathrm{L}$ within 24 hours after injection. The concentration of glargine was then maintained between $260 \pm 134.9 \mathrm{mIU} / \mathrm{L}$ and $188 \pm 55.9 \mathrm{mIU} / \mathrm{L}$ until day 10 Glargine concentrations decreased to $61.5 \pm 16.5 \mathrm{mIU} / \mathrm{L}$ on day 11 and then gradually decreased further thereafter. The blood glucose concentrations were well correlated to the concentration of glargine in the serum. The blood glucose concentrations dropped to a low concentration $(75 \pm 14.5 \mathrm{mg} / \mathrm{dL})$ on the first day after injection and were maintained at the low concentration $(<200 \mathrm{mg} / \mathrm{dL})$ for 9 days before it gradually went back up. Blood glucose went back up to more than $400 \mathrm{mg} / \mathrm{dL}$ on day 13 and then was constantly maintained at normally high concentrations as seen before the injection.

Figure 4-13 shows a comparison of serum glargine concentrations in ZDF rats after subcutaneous injections of 4\% insulin glargine loaded) in PLGA (5\%) gel formulation (Formulation OA-G-4) and PBS (Formulation OA-G-1). There was a significant difference between serum glargine concentrations after subcutaneous injection of formulation of insulin glargine with 4\% loading with PLGA (Formulation OA-G-4) and formulation of insulin glargine with $4 \%$ loading without PLGA (Formulation OA-G1) at $95 \%$ confidence level $(\mathrm{P}=0.001)$ indicating that insulin glargine with $4 \%$ loading with PLGA causes significantly extension in serum glargine concentrations when compared to insulin glargine with $4 \%$ loading without PLGA. It is evident from the figure that the serum glargine concentrations rose rapidly after subcutaneous injection of formulation of insulin glargine with 4\% loading without PLGA (Formulation OA-G-1). The serum glargine concentrations remained above $500 \mathrm{mIU} / \mathrm{L}$ until day 4 . The serum glargine concentrations decreased to near zero levels on day six. However, the serum glargine concentrations rose slowly after subcutaneous injection of formulation of insulin glargine with 4\% loading with PLGA (Formulation OA-G-4). Furthermore, the serum glargine concentrations were above $2500 \mathrm{mIU} / \mathrm{L}$ until day 10.

To further study the effect of presence of zinc sulfate in the PLGA gels loaded with insulin glargine on the biological effect of these gels, three formulations at different concentrations of zinc sulfate were prepared. Figure 4-14 shows the comparison of blood glucose concentrations in ZDF rats after subcutaneous injections of different formulations loaded with $4 \%$ insulin glargine and various zinc sulfate concentrations. It is evident from the figure that addition of zinc sulfate in the formulations lowered the rate of blood glucose lowering effect. At the highest zinc sulfate concentration of $0.5 \%$ (Formulation OA-G-6), the blood glucose concentrations never dropped below $300 \mathrm{mg} / \mathrm{dL}$. Moreover, the blood glucose concentrations remained between 300 to $400 \mathrm{mg} / \mathrm{dL}$ for 14 days. 


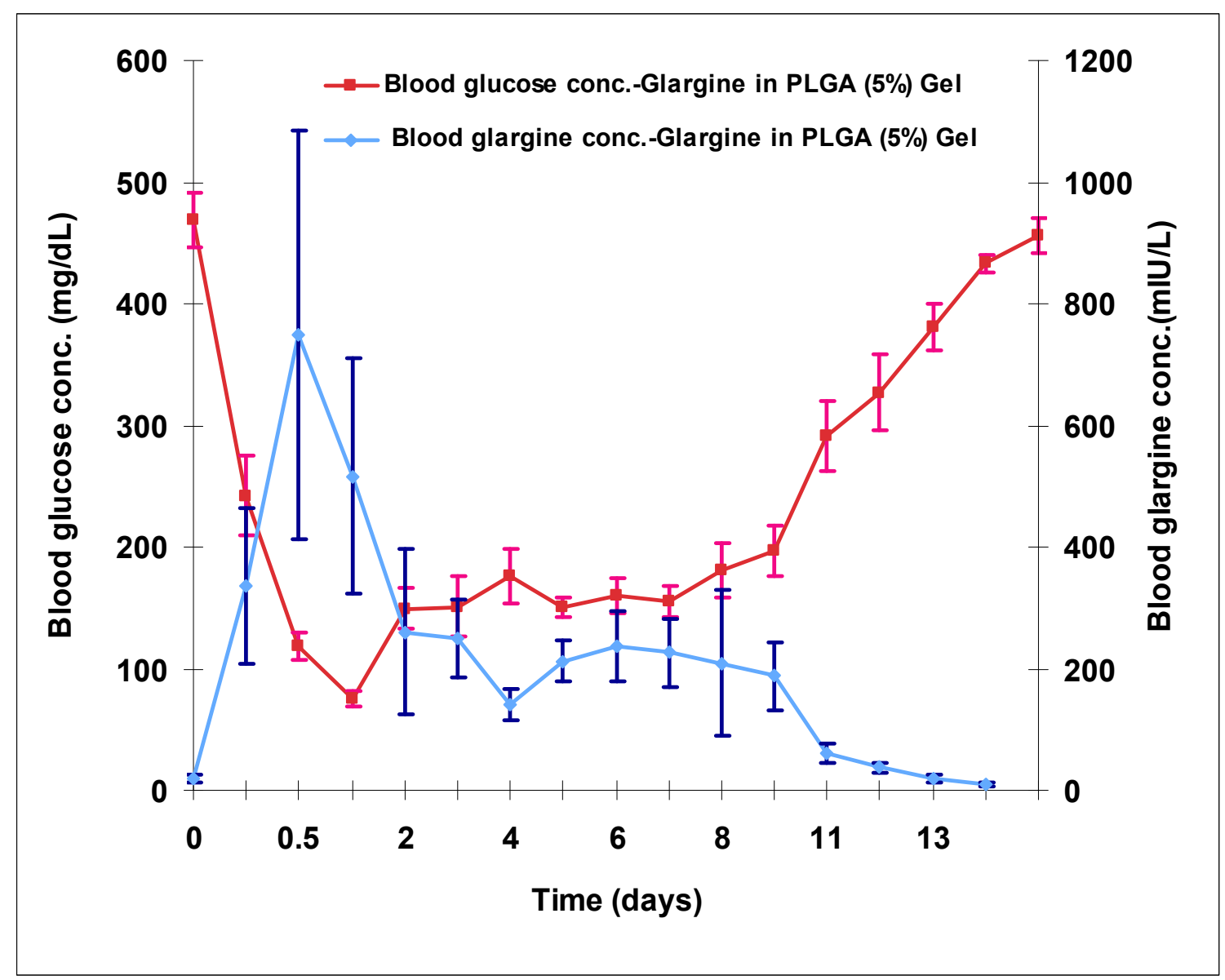

Figure 4-12 Blood glucose concentrations and concentrations of insulin glargine in serum of ZDF rats after a single subcutaneous injection of Formulation OA-G-4 (Mean \pm SEM, $\mathrm{n}=6$ ) 


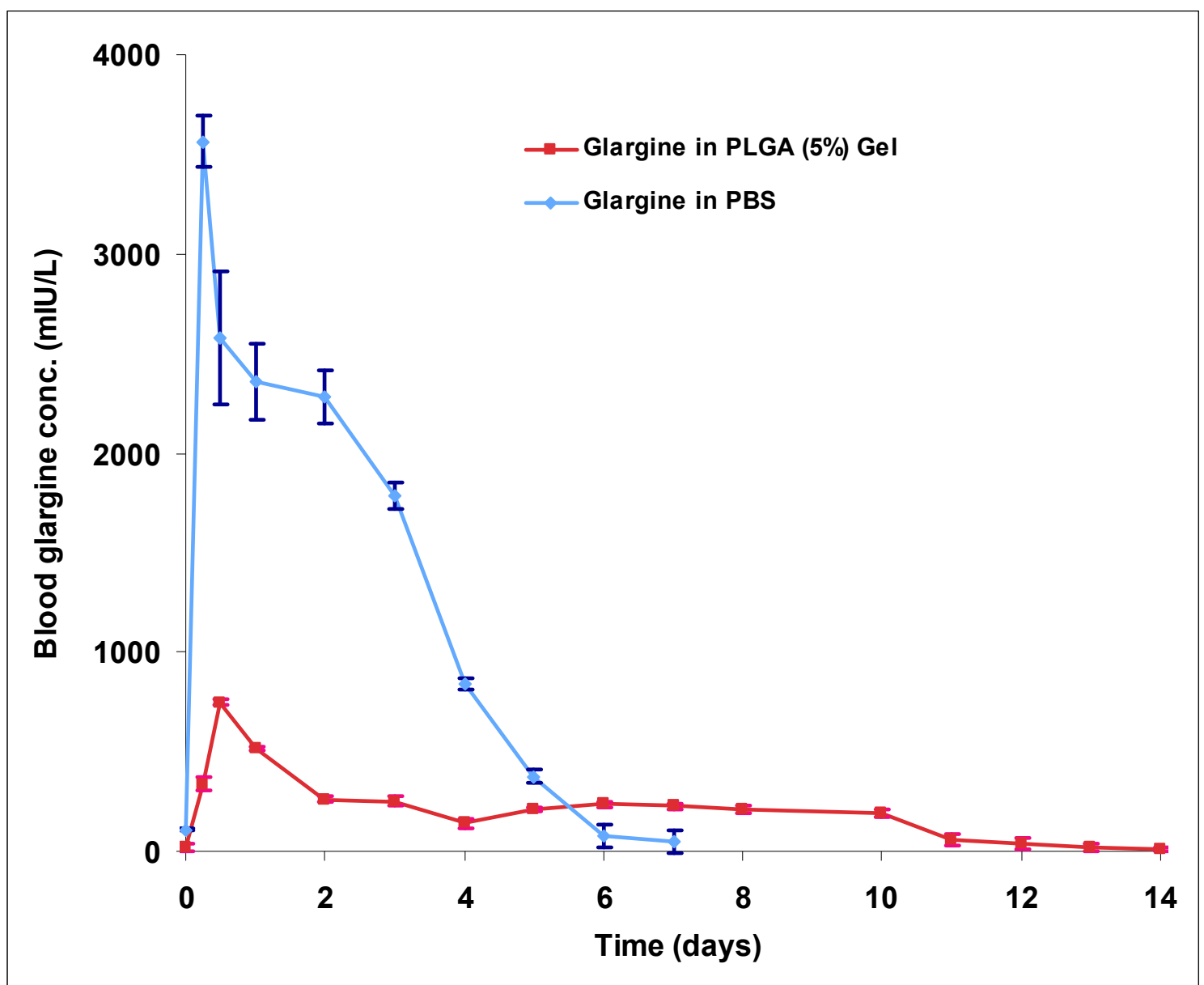

Figure 4-13 Comparison of serum glargine concentrations in ZDF rats after subcutaneous injections of 4\% insulin glargine loaded in PLGA (5\%) gel formulation (Formulation OA-G-4) and PBS 


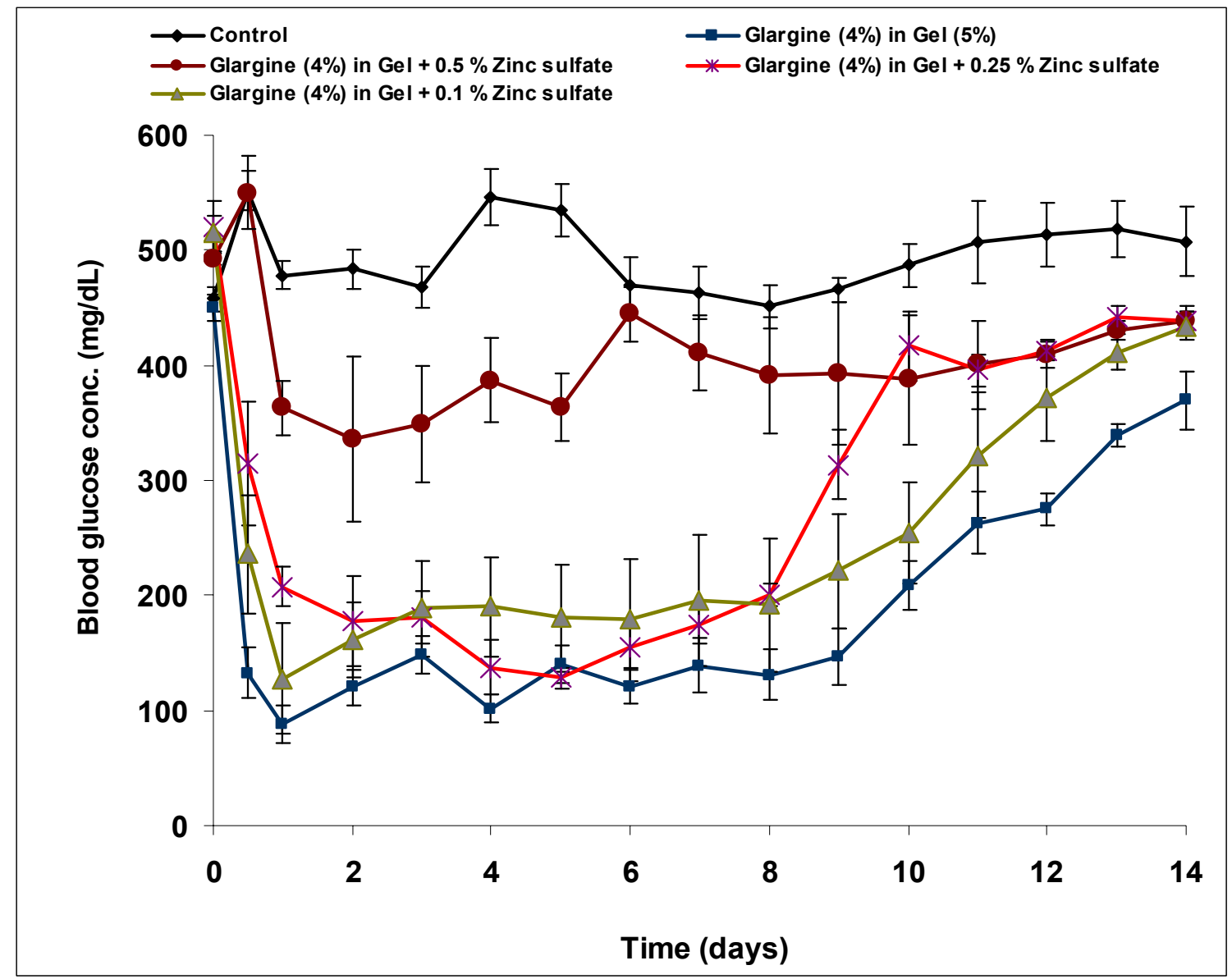

Figure 4-14 Comparison of blood glucose concentrations in ZDF rats after injections of insulin glargine (4\% loading) in different formulations with various zinc sulfate concentrations $($ Mean \pm SEM, $n=5)$ 
There was a significant difference between AUEC after subcutaneous injection of formulation of insulin glargine with $4 \%$ loading with PLGA and zinc sulfate concentration of $0.5 \%$ (Formulation OA-G-6) and control at 95\% confidence level ( $\mathrm{P}=$ 0.02 ) indicating that insulin glargine with $4 \%$ loading with PLGA and zinc sulfate concentration of $0.5 \%$ causes significantly greater reduction in blood glucose when compared to control. Furthermore, There was also a significant difference between AUEC after subcutaneous injection of formulation of insulin glargine with $4 \%$ loading with PLGA (Formulation OA-G-4) and formulation of insulin glargine with $4 \%$ loading with PLGA and zinc sulfate concentration of $0.5 \%$ (Formulation OA-G-6) at 95\% confidence level $(\mathrm{P}=0.002)$ indicating that insulin glargine with $4 \%$ loading with PLGA causes significantly greater reduction in blood glucose when compared to formulation of insulin glargine with $4 \%$ loading with PLGA and zinc sulfate concentration of $0.5 \%$ (Formulation OA-G-6).

As the zinc sulfate concentration was reduced from $0.5 \%$ to $0.25 \%$ (Formulation OA-G-7), the blood glucose concentrations dropped from $520 \mathrm{mg} / \mathrm{dL}$ to about $178 \mathrm{mg} / \mathrm{dL}$ in 2 days, and remained below $200 \mathrm{mg} / \mathrm{dL}$ for 8 days, before rising steadily above 400 $\mathrm{mg} / \mathrm{dL}$ on day 10 . As the zinc sulfate concentration was further reduced from $0.25 \%$ to $0.1 \%$ (Formulation OA-G-8), the blood glucose concentrations dropped from $515 \mathrm{mg} / \mathrm{dL}$ to about $128 \mathrm{mg} / \mathrm{dL}$ in 24 hours, and remained below $200 \mathrm{mg} / \mathrm{dL}$ for 8 days, before rising steadily above $400 \mathrm{mg} / \mathrm{dL}$ on day 13 .

Moreover, no significant difference was observed between AUEC after subcutaneous injection formulation of insulin glargine with 4\% loading with PLGA (Formulation OA-G-4) and formulation of insulin glargine with 4\% loading with PLGA and zinc sulfate concentration of $0.25 \%$ (Formulation OA-G-7) (P>0.05) and zinc sulfate concentration of $0.1 \%$ (Formulation OA-G-8) at $95 \%$ confidence level indicating that formulation of insulin glargine with 4\% loading with PLGA (Formulation OA-G-4) is no better than formulation of insulin glargine with $4 \%$ loading with PLGA and zinc sulfate concentration of $0.25 \%$ (Formulation OA-G-7) and zinc sulfate concentration of $0.1 \%$ (Formulation OA-G-8), in reduction of blood glucose concentrations. Hence, zinc sulfate could be used as a release modifier for insulin glargine in the gels. However, the amount of zinc sulfate in the gel needs to be optimized, so that it can provide a prolonged effect in vivo.

\subsection{Conclusions}

Insulin glargine particles were purified and then freeze-dried from commercial Lantus ${ }^{\circledR}$ formulation. The purity of the freeze-dried particles of insulin glargine was determined using HPLC, and was found to be $94.6 \%$. These insulin glargine particles were suspended in saline and subcutaneously injected in ZDF rats. The bioactivity, as measured by blood glucose lowering effect, was found to be similar to regular human insulin and the marketed formulation called Lantus ${ }^{\circledR}$ The PLGA gel formulations prepared with insulin glargine particles had duration of action of 10 days following a single subcutaneous injection. The concentration of insulin glargine was maintained 
between $260 \pm 134.9 \mathrm{mIU} / \mathrm{L}$ and $188 \pm 55.9 \mathrm{mIU} / \mathrm{L}$ until day 10 after single subcutaneous injection of some of these formulations and he corresponding blood glucose concentrations were suppressed from above $400 \mathrm{mg} / \mathrm{dL}$ to below $200 \mathrm{mg} / \mathrm{dL}$ from day 1 to day 10 post injection. The addition of zinc sulfate to the formulations prepared with purified insulin glargine particles further slowed down the drop in blood glucose concentrations. 


\section{LIST OF REFERENCES}

1. Definition, Diagnosis and Classification of Diabetes Mellitus and Its Complications. World Health Organization; 1999. http://www.diabetes.com.au/pdf/who_report.pdf. Last accessed: July 1st, 2008.

2. Colagiuri S, Borch-Johnsen K, Glumer C, Vistisen D. There really is an epidemic of type 2 diabetes. Diabetologia. 2005 Jul 9.

3. Krall LP, Levine R, Barnett D. The history of diabetes. In: Kahn CR, Weir GC, editors. Joslin's diabetes mellitus. 13th ed. Philadelphia: Lea and Febiger; 1994. p. 1-14.

4. Banting F, Best C. Pancreatic extracts. J lab Clin Med. 1922; 1990 (115):25472.

5. Ringertz JLNR. The Nobel Prize in Physiology or Medicine, 1901-2000. Nobelprizeorg. 26 June 2001

6. Graves PM, Eisenbarth GS. Pathogenesis, prediction and trials for the prevention of insulin-dependent (type 1) diabetes mellitus. Advanced Drug Delivery Reviews. 1999;35 (2,3):143-56.

7. Kaprio J, Tuomilehto J, Koskenvuo M, Romanov K, Reunanen A, Eriksson J, et al. Concordance for type 1 (insulin-dependent) and type 2 (non-insulindependent) diabetes mellitus in a population-based cohort of twins in Finland. Diabetologia. 1992; 35 (11):1060-7.

8. Kyvik KO, Green A, Beck-Nielsen H. Concordance rates of insulin dependent diabetes mellitus: a population based study of young Danish twins. BMJ (Clinical research ed). 1995; 311 (7010):913-7.

9. Rewers M, Bugawan TL, Norris JM, Blair A, Beaty B, Hoffman M, et al. Newborn screening for HLA markers associated with IDDM: diabetes autoimmunity study in the young (DAISY). Diabetologia. 1996; 39 (7):807-12.

10. Davies JL, Kawaguchi Y, Bennett ST, Copeman JB, Cordell HJ, Pritchard LE, et al. A genome-wide search for human type 1 diabetes susceptibility genes. Nature (London, United Kingdom). 1994;371 (6493):130-6.

11. Borch-Johnsen K, Joner G, Mandrup-Poulsen T, Christy M, ZachauChristiansen B, Kastrup K, et al. Relation between breast-feeding and incidence rates of insulin-dependent diabetes mellitus. A hypothesis. Lancet. 1984;2 (8411):1083-6. 
12. Graves PM, Norris JM, Pallansch MA, Gerling IC, Rewers M. The role of enteroviral infections in the development of IDDM: limitations of current approaches. Diabetes. 1997;46 (2):161-8.

13. Clements GB, Galbraith DN, Taylor KW. Coxsackie B virus infection and onset of childhood diabetes. Lancet. 1995;346 (8969):221-3.

14. Frisk G, Friman G, Tuvemo T, Fohlman J, Diderholm H. Coxsackie B virus IgM in children at onset of type 1 (insulin-dependent) diabetes mellitus: evidence for IgM induction by a recent or current infection. Diabetologia. 1992;35 (3):249-53.

15. Hyoty H, Hiltunen M, Knip M, Laakkonen M, Vahasalo P, Karjalainen J, et al. A prospective study of the role of coxsackie $\mathrm{B}$ and other enterovirus infections in the pathogenesis of IDDM. Childhood Diabetes in Finland (DiMe) Study Group. Diabetes. 1995;44 (6):652-7.

16. Eberhardt MS, Wagener DK, Orchard TJ, LaPorte RE, Cavender DE, Rabin BS, et al. HLA heterogeneity of insulin-dependent diabetes mellitus at diagnosis. The Pittsburgh IDDM study. Diabetes. 1985;34 (12):1247-52.

17. Akerblom HK, Savilahti E, Saukkonen TT, Paganus A, Virtanen SM, Teramo $\mathrm{K}$, et al. The case for elimination of cow's milk in early infancy in the prevention of type 1 diabetes: the Finnish experience. Diabetes Metab Rev 1993;9 (4):269-78.

18. Jun H-S, Bae HY, Lee BR, Koh KS, Kim YS, Lee KW, et al. Pathogenesis of non-insulin-dependent (type II) diabetes mellitus (NIDDM) - genetic predisposition and metabolic abnormalities. Advanced Drug Delivery Reviews. $1999 ; 35(2,3): 157-77$.

19. Bach JF. Insulin-dependent diabetes mellitus as a beta-cell targeted disease of immunoregulation. Journal of autoimmunity. 1995;8 (4):439-63.

20. Yki-Jarvinen H. Pathogenesis of non-insulin-dependent diabetes mellitus. Lancet. 1994;343 (8889):91-5.

21. DeFronzo RA. Lilly lecture 1987. The triumvirate: beta-cell, muscle, liver. A collusion responsible for NIDDM. Diabetes. 1988;37 (6):667-87.

22. Del Prato S, Matsuda M, Simonson DC, Groop LC, Sheehan P, Leonetti F, et al. Studies on the mass action effect of glucose in NIDDM and IDDM. Evidence for glucose resistance. Diabetologia. 1997;40 (6):687-97.

23. Bogardus C, Lillioja S, Howard BV, Reaven G, Mott D. Relationships between insulin secretion, insulin action, and fasting plasma glucose concentration in 
nondiabetic and noninsulin-dependent diabetic subjects. Journal of Clinical Investigation. 1984;74 (4):1238-46.

24. Firth R, Bell P, Rizza R. Insulin action in non-insulin-dependent diabetes mellitus: the relationship between hepatic and extrahepatic insulin resistance and obesity. Metabolism: clinical and experimental. 1987;36 (11):1091-5.

25. Consoli A, Nurjhan N, Capani F, Gerich J. Predominant role of gluconeogenesis in increased hepatic glucose production in NIDDM. Diabetes. 1989;38 (5):550-7.

26. Nurjhan N, Consoli A, Gerich J. Increased lipolysis and its consequences on gluconeogenesis in non-insulin-dependent diabetes mellitus. J Clin Invest. 1992;89 (1):169-75.

27. Tayek JA, Katz J. Glucose production, recycling, and gluconeogenesis in normals and diabetics: a mass isotopomer [U-13C]glucose study. American Journal of Physiology. 1996;270 (4, Pt. 1):E709-E17.

28. Murnaghan JH, Talalay P. John Jacob Abel and the crystallization of insulin. Perspect Biol Med. 1967 Spring;10 (3):334-80.

29. Scott DA, Fisher AM. The effect of zinc salts on the action of insulin. J Pharmacol. 1935;55:206-21.

30. Scott DA, Fisher AM. Crystalline insulin. Biochemical Journal. 1935;29:104854.

31. Scott DA. Crystalline insulin. Biochemical Journal. 1934;28:1592-602.

32. Hagedorn H, Jensen B, Krarup N, Wodstrup I. Protamine insulinate. JAMA. 1936;106:177-80.

33. Scott DA, Fisher AM, inventors; (Governors of The University of Toronto). assignee. Therapeutic composition containing insulin. US patent 2179384. 1939.

34. Scott DA, Fisher AM. The preparation of insulin. Proceedings and Transactions of the Royal Society of Canada. 1938;32, V:59-63.

35. Hallas-Moller K, Petersen K, Schlichtkrull J. Combinaciones cristalinas y amorfas de insulina-zinc de accion prolongada. El Dia medico. 1954;26 (29):720-3.

36. Hallas-Moller K, Jersild M, Petersen K, Schlicht-Krull J. Studies on new insulin preparations with retarded effect. Nordisk medicin. 1953;50 (27):949. 
37. Hallas M, Oller K, Jersild M, Petersen K, Schlichtkrull J. Zinc insulin preparations for single daily injection; clinical studies of new preparations with prolonged action. Journal of the American Medical Association. 1952;150 (17):1667-71.

38. Hallas M, Oller K, Petersen K, Schlichtkrull J. Crystalline and amorphous insulin-zinc compounds with prolonged action. Science (New York, NY). 1952;116 (3015):394-8.

39. Hallas-Moller K, Jersild M, Petersen K, Schlichtkrull J. Clinical studies on new insulin preparations with prolonged action; one daily injection with insulin zinc preparations. Ugeskrift for laeger. 1951;113 (52):1767-71.

40. Hallas-Moller K, Petersen K, Schlichtkrull J. Crystalline and amorphous insulin and zinc compounds with prolonged action. Ugeskrift for laeger. $1951 ; 113(52): 1761-7$.

41. Ryle AP, Sanger F, Smith LF, Kitai R. The disulphide bonds of insulin. Biochem Journal. 1955;60:541-56.

42. Sanger F. Chemistry of insulin: determination of the structure of insulin opens the way to greater understanding of life process. Science. 1959;129:1340-4.

43. Andersson BGMB. The Nobel Prize in Chemistry: The Development of Modern Chemistry. Nobelprizeorg. 2001.

44. Meienhofer J, Schnabel E, Bremer H, Brinkhoff O, Zabel R, Sroka W, et al. Synthesis of the insulin chain and the combination to insulin active preparation. Zeitschrift fuer Naturforschung. 1963;18b (12):1120-1.

45. Gillessen D, Schnabel E, Meienhofer J. Peptides. XXX. Synthesis of the insulin sequence B 13-30. Ann. 1963;667:164-71.

46. Meienhofer J, Brinkhof O. Preparation of an insulin A chain derivative with an intact intrachain disulfide bond. Nature (London, United Kingdom). 1963;199 (4898):1095-6.

47. Meienhofer J, Schnabel E, Bremer H, Brinkhoff O, Zabel R, Sroka W, et al. Synthesis of Insulin Chains and Their Combination to Insulin-Active Preparations. Zeitschrift fur Naturforschung Teil B: Chemie, Biochemie, Biophysik, Biologie. 1963;18:1120-1.

48. Katsoyannis PG, Tilak M. Insulin peptides. VIII. A synthetic heptadecapeptide derivative corresponding to the $\mathrm{C}$-terminal sequence of the B-chain of insulin. Journal of the American Chemical Society. 1963;85 (24):4028-31. 
49. Katsoyannis PG, Tometsko A, Fukuda K. Insulin peptides. IX. The synthesis of the A-chain of insulin and its combination with natural B-chain to generate insulin activity. Journal of the American Chemical Society. 1963;85 (18):28635.

50. Kung Y-Ti, et al. Preparation of crystalline insulin. Kexue Tongbao (Chinese Edition). 1966 (11):941-5.

51. Kung YT, Du YC, Huang WT, Chen CC, Ke LT. Total synthesis of crystalline insulin. Scientia Sinica. 1966;15 (4):544-61.

52. Niu CI, Kung YT, Huang WT, Ke LT, Chen CC. Synthesis of crystalline insulin from its natural A-chain and the synthetic B-chain. Scientia Sinica. $1966 ; 15$ (2):231-44.

53. Kung YT, Ke LT, Niu C. Synthesis of the peptide fragments of the B-chain of insulin. X. Synthesis of a derivative of the C-terminal docosapeptide of the Bchain of insulin. Scientia Sinica. 1966;15 (2):221-30.

54. Steiner DF, Cunningham D, Spigelman L, Aten B. Insulin biosynthesis: evidence for a precursor. Science (New York, NY). 1967;157 (789):697-700.

55. Steiner DF, Oyer PE. Biosynthesis of insulin and a probable precursor of insulin by a human islet-cell adenoma. Proceedings of the National Academy of Sciences of the United States of America. 1967;57 (2):473-80.

56. Adams MJ, Dodson GG, Dodson E, Hodgkin DC. Report on recent calculations on rhombohedral insulin crystals containing lead. Conform Biopolym, Pap Int Symp. 1967;1:9-16.

57. Morihara K, Oka T, Tsuzuki H, Inouye K, Sakakibara S. Semisynthesis of human insulin: trypsin-catalyzed replacement of alanine B30 by threonine in porcine insulin. Pept, Struct Biol Funct, Proc Am Pept Symp, 6th. 1979:61720.

58. Inouye K, Watanabe K, Morihara K, Tochino Y, Kanaya T, Emura J, et al. Enzyme-assisted semisynthesis of human insulin. Peptide Chemistry. 1979;16th:141-6.

59. Inouye K, Watanabe K, Morihara K, Tochino Y, Kanaya T, Emura J, et al. Enzyme-assisted semisynthesis of human insulin. Journal of the American Chemical Society. 1979;101 (3):751-2.

60. Goeddel DV, Kleid DG, Bolivar F, Heyneker HL, Yansura DG, Crea R, et al. Expression in Escherichia coli of chemically synthesized genes for human insulin. Proc Natl Acad Sci U S A. 1979 Jan;76 (1):106-10. 
61. Thayer A. The top pharmaceuticals that changed the world. Chem Eng News. $2005 ; 83(25)$.

62. Beals JM, Kovach PM. Insulin. In: Crommelin DJ, Sindelar RD, editors. Pharmaceutical Biotechnology: An introduction for pharmacists and pharmaceutical scientists. 1 ed. Amsterdam: Harwood Academic Publishers; 1997. p. 229-38.

63. Ciszak E, Beals JM, Frank BH, Baker JC, Carter ND, Smith GD. Role of Cterminal B-chain residues in insulin assembly: the structure of hexameric LysB28ProB29-human insulin. Structure. 1995 Jun 15;3 (6):615-22.

64. Pullen RA, Lindsay DG, Wood SP, Tickle IJ, Blundell TL, Wollmer A, et al. Receptor-binding region of insulin. Nature (London, United Kingdom). 1976;259 (5542):369-73.

65. Brandenburg D, Busse WD, Gattner HG, Zahn H, Wollmer A, Gliemann J, et al. Structure-function studies with chemically modified insulins. Pept, Proc Eur Pept Symp, 12th. 1973:270-83.

66. Derewenda U, Dodson GG. The structure and sequence of insulin. Mol Struct Biol. 1993:260-77.

67. Derewenda U, Derewenda Z, Dodson GG, Hubbard RE, Korber F. Molecular structure of insulin: the insulin monomer and its assembly. British Medical Bulletin. 1989;45 (1):4-18.

68. Bentley GA, Brange J, Derewenda Z, Dodson EJ, Dodson GG, Markussen J, et al. Role of B13 Glu in insulin assembly. The hexamer structure of recombinant mutant (B13 Glu -> Gln) insulin. Journal of Molecular Biology. 1992;228 (4):1163-76.

69. Derewenda U, Derewenda Z, Dodson GG, Hubbard RE, Korber F. Molecular structure of insulin: the insulin monomer and its assembly. British Medical Bulletin. 1989;45 (1):4-18.

70. Smith GD, Swenson DC, Derewenda Z, Dodson EJ, Dodson GG, Reynolds $\mathrm{CD}$, et al. The structure of a new hexameric form of human insulin. Pept: Chem Biol, Proc Am Pept Symp 10th. 1988:44-6.

71. Brange J, Langkjoer L. Insulin structure and stability. Pharmaceutical biotechnology. 1993;5:315-50.

72. Hassiepen U, Federwisch M, Mulders T, Wollmer A. The lifetime of insulin hexamers. Biophysical journal. 1999;77 (3):1638-54. 
73. Schlichtkrull J, Noring IM, inventors; (Novo Terapeutisk Laboratorium A/S). assignee. Crystallization of insulin using freeze-dried insulin as seeding material. US patent 2819999. 1958.

74. Olsen HB, Ludvigsen S, Kaarsholm NC. Solution Structure of an Engineered Insulin Monomer at Neutral pH. Biochemistry. 1996;35 (27):8836-45.

75. Kadima W. Role of Metal Ions in the T- To R-Allosteric Transition in the Insulin Hexamer. Biochemistry. 1999;38 (41):13443-52.

76. Derewenda U, Derewenda Z, Dodson EJ, Dodson GG, Reynolds CD, Smith GD, et al. Phenol stabilizes more helix in a new symmetrical zinc insulin hexamer. Nature. 1989 Apr 13;338 (6216):594-6.

77. Coffman FD, Dunn MF. Insulin-metal ion interactions: the binding of divalent cations to insulin hexamers and tetramers and the assembly of insulin hexamers. Biochemistry. 1988;27 (16):6179-87.

78. Kaarsholm NC, Ko HC, Dunn MF. Comparison of solution structural flexibility and zinc binding domains for insulin, proinsulin, and miniproinsulin. Biochemistry. 1989;28 (10):4427-35.

79. Schlichtkrull J, inventor (Novo Terapeutisk Laboratorium A/S). assignee. Insulin preparations. GB patent 860515. 1961.

80. Roy M, Lee RWK, Brange J, Dunn MF. Proton NMR spectrum of the native human insulin monomer. Evidence for conformational differences between the monomer and aggregated forms. Journal of Biological Chemistry. 1990;265 (10):5448-52.

81. Wollmer A, Rannefeld B, Johansen BR, Hejnaes KR, Balschmidt P, Hansen FB. Phenol-promoted structural transformation of insulin in solution. Biological Chemistry Hoppe-Seyler. 1987;368 (8):903-11.

82. Hallas-Moller K, Petersen K, Schlichtkrull J, inventors; (Novo Terapeutisk Laboratorium A/S). assignee. Injectable insulin with depot action. DE patent 950595. 1956.

83. Schlichtkrull J, Noring IM, inventors; (Novo Terapeutisk Laboratorium A/S). assignee. Crystallization of insulin using freeze-dried insulin as seeding material. DE patent 952208. 1956.

84. Schlichtkrull J. Insulin crystals. II. Shape of rhomboidal zinc-insulin crystals in relation to species and crystallization media. Acta Chemica Scandinavica. 1956;10:1459-64. 
85. Schlichtkrull J. Insulin crystals. I. The minimum mole fraction of metal in insulin crystals prepared with $\mathrm{Zn}++, \mathrm{Cd}++, \mathrm{Co}++, \mathrm{Ni}++, \mathrm{Cu}++, \mathrm{Mn}++$, or $\mathrm{Fe}++$. Acta Chemica Scandinavica. 1956;10:1455-8.

86. Blundell T, Dodson G, Hodgkin D, Mercola D. Insulin. Structure in the crystal and its reflection in chemistry and biology. Advances in Protein Chemistry. 1972;26:279-402.

87. Hallas-Moller K. Chemical, biological, and physiological background of the new insulin-zinc suspensions. Lancet. 1954;267:1029-34.

88. White MF. The IRS-signaling system: a network of docking proteins that mediate insulin and cytokine action. Recent Prog Horm Res. 1998;53:119-38.

89. White MF. The IRS-signalling system: a network of docking proteins that mediate insulin action. Mol Cell Biochem. 1998 May;182 (1-2):3-11.

90. Maturo JM, 3rd, Hollenberg MD. Insulin receptor: interaction with nonreceptor glycoprotein from liver cell membranes. Proc Natl Acad Sci U S A. 1978 Jul;75 (7):3070-4.

91. Etherton TD. The role of insulin-receptor interactions in regulation of nutrient utilization by skeletal muscle and adipose tissue: a review. J Anim Sci. 1982 Jan;54 (1):58-67.

92. Boute N, Pernet K, Issad T. Monitoring the activation state of the insulin receptor using bioluminescence resonance energy transfer. Mol Pharmacol. 2001 Oct;60 (4):640-5.

93. Duckworth WC, Bennett RG, Hamel FG. Insulin degradation: progress and potential. Endocr Rev. 1998 Oct;19 (5):608-24.

94. Morishima T, Pye S, Bradshaw C, Radziuk J. Posthepatic rate of appearance of insulin: measurement and validation in the nonsteady state. Am J Physiol. 1992 Oct;263 (4 Pt 1):E772-9.

95. Fawcett J, Rabkin R. Endosomes degrade insulin. Contrib Nephrol. 1993;101:61-5.

96. Sato H, Terasaki T, Mizuguchi H, Okumura K, Tsuji A. Receptor-recycling model of clearance and distribution of insulin in the perfused mouse liver. Diabetologia. 1991 Sep;34 (9):613-21.

97. Duckworth WC, Runyan KR, Wright RK, Halban PA, Solomon SS. Insulin degradation by hepatocytes in primary culture. Endocrinology. 1981 Apr;108 (4):1142-7. 
98. Duckworth WC, Hamel FG, Peavy DE. Hepatic metabolism of insulin. Am J Med. 1988 Nov 28;85 (5A):71-6.

99. Rabkin R, Kitaji J. Renal metabolism of peptide hormones. Miner Electrolyte Metab. 1983;9 (4-6):212-26.

100. Fawcett J, Rabkin R. Degradation of insulin by isolated rat renal cortical endosomes. Endocrinology. 1993 Oct;133 (4):1539-47.

101. Sato H, Yoshioka K, Terasaki T, Tsuji A. Receptor-mediated endocytosis of A14-125I-insulin by the nonfiltering perfused rat kidney. Biochim Biophys Acta. 1991 Apr 9;1073 (3):442-50.

102. Clot JP, Janicot M, Fouque F, Desbuquois B, Haumont PY, Lederer F. Characterization of insulin degradation products generated in liver endosomes: in vivo and in vitro studies. Mol Cell Endocrinol. 1990 Sep 10;72 (3):175-85.

103. Savoy LA, Jones RM, Pochon S, Davies JG, Muir AV, Offord RE, et al. Identification by fast atom bombardment mass spectrometry of insulin fragments produced by insulin proteinase. Biochem J. 1988 Jan 1;249 (1):21522.

104. Davies JG, Muir AV, Rose K, Offord RE. Identification of radioactive insulin fragments liberated by insulin proteinase during the degradation of semisynthetic [3H]GlyA1]insulin and [3H]PheB1]insulin. Biochem J. 1988 Jan 1;249 (1):209-14.

105. Davies JG, Muir AV, Offord RE. Identification of some cleavage sites of insulin by insulin proteinase. Biochem J. 1986 Dec 1;240 (2):609-12.

106. Muller D, Schulze C, Baumeister H, Buck F, Richter D. Rat insulin-degrading enzyme: cleavage pattern of the natriuretic peptide hormones ANP, BNP, and CNP revealed by HPLC and mass spectrometry. Biochemistry. 1992 Nov 17;31 (45):11138-43.

107. Stentz FB, Harris HL, Kitabchi AE. Characterization of insulin-degrading activity of intact and subcellular components of human fibroblasts. Endocrinology. 1985 Mar;116 (3):926-34.

108. Buffington CK, el-Shiekh T, Kitabchi AE, Matteri R. Phytohemagglutinin (PHA) activated human T-lymphocytes: concomitant appearance of insulin binding, degradation and insulin-mediated activation of pyruvate dehydrogenase (PDH). Biochem Biophys Res Commun. 1986 Jan 14;134 (1):412-9. 
109. Stentz FB, Kitabchi AE, Schilling JW, Schronk LR, Seyer JM. Identification of insulin intermediates and sites of cleavage of native insulin by insulin protease from human fibroblasts. J Biol Chem. 1989 Dec 5;264 (34):20275-82.

110. Duckworth WC, Heinemann MA, Kitabchi AE. Purification of insulin-specific protease by affinity chromatography. Proc Natl Acad Sci U S A. 1972 Dec;69 (12):3698-702.

111. Nuss JM, Wagman AS. Chapter 19. Recent advances in therapeutic approaches to type 2 diabetes. Annual Reports in Medicinal Chemistry. 2000;35:211-20.

112. Ahmann AJ, Riddle MC. Insulin therapy in type 2 diabetes mellitus. Insulin Therapy. 2002:113-25.

113. Chen Q. Controlled release of insulin from a novel biodegradable injectable gel system.2006. Doctoral Dissertation; Electronoc Resources http://sunzi1.lib.hku.hk. Last accessed: October 30th, 2007.

114. Garber AJ. Benefits of combination therapy of insulin and oral hypoglycemic agents. Archives of Internal Medicine. 2003;163 (15):1781-2.

115. Ratner R, Whitehouse F, Fineman MS, Strobel S, Shen L, Maggs DG, et al. Adjunctive therapy with pramlintide lowers $\mathrm{HbAlc}$ without concomitant weight gain and increased risk of severe hypoglycemia in patients with type 1 diabetes approaching glycemic targets. Experimental and Clinical Endocrinology \& Diabetes. 2005;113 (4):199-204.

116. Weyer C, Fineman MS, Strobel S, Shen L, Data J, Kolterman OG, et al. Properties of pramlintide and insulin upon mixing. American Journal of Health-System Pharmacy. 2005;62 (8):816-22.

117. Ceriello A, Piconi L, Quagliaro L, Wang Y, Schnabel CA, Ruggles JA, et al. Effects of pramlintide on postprandial glucose excursions and measures of oxidative stress in patients with type 1 diabetes. Diabetes Care. 2005;28 (3):632-7.

118. Goldstein DE, Little RR, Lorenz RA, Malone JI, Nathan DM, Peterson CM. Tests of glycemia in diabetes. Diabetes Care. 2004 Jan;27 Suppl 1:S91-3.

119. Rohlfing CL, Wiedmeyer H-M, Little RR, England JD, Tennill A, Goldstein DE. Defining the relationship between plasma glucose and HbAlc: Analysis of glucose profiles and $\mathrm{HbAlc}$ in the Diabetes Control and Complications Trial. Diabetes Care. 2002;25 (2):275-8.

120. Soran H, Younis N. Insulin detemir: a new basal insulin analogue. Diabetes, Obesity and Metabolism. 2006;8 (1):26-30. 
121. Barnett AH. A review of basal insulins. Diabetic Medicine. 2003;20 (11):87385.

122. Kurtzhals P, Schaffer L, Sorensen A, Kristensen C, Jonassen I, Schmid C, et al. Correlations of receptor binding and metabolic and mitogenic potencies of insulin analogs designed for clinical use. Diabetes. 2000;49 (6):999-1005.

123. Simpson D, McCormack PL, Keating GM, Lyseng-Williamson KA. Insulin lispro a review of its use in the management of diabetes mellitus. Drugs. 2007;67 (3):407-34.

124. Binder C, Brange J. Insulin chemistry and pharmacokinetics. In: Porte D, Roberts SS, Baron A, editors. Ellenbery \& Rifkin's Diabetes Mellitus. 6th ed: McGraw-Hill; 2003.

125. Birnbaum DT, Kilcomons MA, DeFelippis MR, Beals JM. Assembly and dissociation of human insulin and LysB28ProB29-insulin hexamers: a comparison study. Pharmaceutical research. 1997;14 (1):25-36.

126. Anonymous. Insulin aspart. Asp-B28, NovoRapid. Drugs in R\&D. 1999;2 (2):103-6.

127. Robinson DM, Wellington K. Insulin glulisine. Drugs. 2006;66 (6):861-9.

128. Levien TL, Baker DE, White JR, Jr., Campbell RK. Insulin glargine: A new basal insulin. Annals of Pharmacotherapy. 2002;36 (6):1019-27.

129. Gillies PS, Figgitt DP, Lamb HM. Insulin glargine. Drugs. 2000;59 (2):253-60.

130. Morales J. Defining the role of insulin detemir in basal insulin therapy. Drugs. 2007;67 (17):2557-84.

131. Jonassen I, Havelund S, Ribel U, Plum A, Loftager M, Hoeg-Jensen T, et al. Biochemical and Physiological Properties of a Novel Series of Long-Acting Insulin Analogs Obtained by Acylation with Cholic Acid Derivatives. Pharmaceutical Research. 2006;23 (1):49-55.

132. Kurtzhals P, Havelund S, Jonassen I, Markussen J. Effect of Fatty Acids and Selected Drugs on the Albumin Binding of a Long-Acting, Acylated Insulin Analog. Journal of Pharmaceutical Sciences. 1997;86 (12):1365-8.

133. Whittingham JL, Havelund S, Jonassen I. Crystal Structure of a ProlongedActing Insulin with Albumin-Binding Properties. Biochemistry. 1997;36 (10):2826-31. 
134. Kurtzhals P, Havelund S, Jonassen I, Markussen J. Effect of fatty acids and selected drugs on the albumin binding of a long-acting, acylated insulin analogue. Journal of pharmaceutical sciences. 1997;86 (12):1365-8.

135. Whittingham JL, Havelund S, Jonassen I. Crystal structure of a prolongedacting insulin with albumin-binding properties. Biochemistry. 1997;36 (10):2826-31.

136. Khafagy E-S, Morishita M, Onuki Y, Takayama K. Current challenges in noninvasive insulin delivery systems: A comparative review. Advanced Drug Delivery Reviews. 2007;59 (15):1521-46.

137. Gwinup G, Elias AN, Vaziri ND. A case for oral insulin therapy in the prevention of diabetic micro- and macroangiopathy. The International journal of artificial organs. 1990;13 (7):393-5.

138. Kennedy FP. Recent developments in insulin delivery techniques. Current status and future potential. Drugs. 1991;42 (2):213-27.

139. Owens DR. New horizons - alternative routes for insulin therapy. Nature Reviews Drug Discovery. 2002;1 (7):529-40.

140. Cefalu WT. Evolving strategies for insulin delivery and therapy. Drugs. 2004;64 (11):1149-61.

141. Torchilin VP, Lukyanov AN. Peptide and protein drug delivery to and into tumors: challenges and solutions. Drug Discovery Today. 2003;8 (6):259-66.

142. Marschutz MK, Bernkop-Schnurch A. Oral peptide drug delivery: polymerinhibitor conjugates protecting insulin from enzymatic degradation in vitro. Biomaterials. 2000;21 (14):1499-507.

143. Nakamura K, Murray RJ, Joseph JI, Peppas NA, Morishita M, Lowman AM. Oral insulin delivery using P(MAA-g-EG) hydrogels: effects of network morphology on insulin delivery characteristics. Journal of Controlled Release. 2004;95 (3):589-99.

144. Lane ME, O'Driscoll CM, Corrigan OI. Quantitative estimation of the effects of bile salt surfactant systems on insulin stability and permeability in the rat intestine using a mass balance model. Journal of Pharmacy and Pharmacology. 2005;57 (2):169-75.

145. Uchiyama T, Sugiyama T, Quan Y-S, Kotani A, Okada N, Fujita T, et al. Enhanced permeability of insulin across the rat intestinal membrane by various absorption enhancers: their intestinal mucosal toxicity and absorption- 
enhancing mechanism of n-lauryl-b-D-maltopyranoside. Journal of Pharmacy and Pharmacology. 1999;51 (11):1241-50.

146. Morishita M, Kajita M, Suzuki A, Takayama K, Chiba Y, Tokiwa S, et al. The dose-related hypoglycemic effects of insulin emulsions incorporating highly purified EPA and DHA. International Journal of Pharmaceutics. 2000;201 (2):175-85.

147. Morishita M, Morishita I, Takayama K, Machida Y, Nagai T. Site-dependent effect of aprotinin, sodium caprate, disodium-EDTA and sodium glycocholate on intestinal absorption of insulin. Biological \& Pharmaceutical Bulletin. 1993;16 (1):68-72.

148. Majuru S. Development of an oral insulin solid dosage formulation using Emisphere's eligen technology. Drug Delivery Technology. 2005;5 (9):74, 6-8.

149. Majuru S. Advances in the oral delivery of heparin from solid dosage forms using emisphere's eligen oral drug delivery technology. Drug Delivery Technology. 2004;4 (8):84,6-9.

150. Morishita M, Goto T, Nakamura K, Lowman AM, Takayama K, Peppas NA. Novel oral insulin delivery systems based on complexation polymer hydrogels: Single and multiple administration studies in type 1 and 2 diabetic rats. Journal of Controlled Release. 2006;110 (3):587-94.

151. Kim BY, Jeong JH, Park K, Kim JD. Bioadhesive interaction and hypoglycemic effect of insulin-loaded lectin-microparticle conjugates in oral insulin delivery system. J Control Release. 2005 Feb 16;102 (3):525-38.

152. Pan Y, Zheng J-M, Zhao H-Y, Li Y-J, Xu H, Wei G. Relationship between drug effects and particle size of insulin-loaded bioadhesive microspheres. Acta Pharmacologica Sinica. 2002;23 (11):1051-6.

153. Pan Y, Li Y-j, Zhao H-y, Zheng J-m, Xu H, Wei G, et al. Bioadhesive polysaccharide in protein delivery system: chitosan nanoparticles improve the intestinal absorption of insulin in vivo. International Journal of Pharmaceutics. 2002;249 (1-2):139-47.

154. Krauland AH, Guggi D, Bernkop-Schnurch A. Oral insulin delivery: the potential of thiolated chitosan-insulin tablets on non-diabetic rats. Journal of Controlled Release. 2004;95 (3):547-55.

155. Bernkop-Schnurch A, Clausen AE. Biomembrane permeability of peptides: strategies to improve their mucosal uptake. Mini-Reviews in Medicinal Chemistry. 2002;2 (4):295-305. 
156. Watnasirichaikul S, Rades T, Tucker IG, Davies NM. In-vitro release and oral bioactivity of insulin in diabetic rats using nanocapsules dispersed in biocompatible microemulsion. Journal of Pharmacy and Pharmacology. 2002;54 (4):473-80.

157. Kisel MA, Kulik LN, Tsybovsky IS, Vlasov AP, Vorob'yov MS, Kholodova EA, et al. Liposomes with phosphatidylethanol as a carrier for oral delivery of insulin: studies in the rat. International Journal of Pharmaceutics. 2001;216 (12):105-14.

158. Jones MC, Leroux JC. Polymeric micelles - a new generation of colloidal drug carriers. European Journal of Pharmaceutics and Biopharmaceutics. 1999;48 (2):101-11.

159. Toorisaka E, Hashida M, Kamiya N, Ono H, Kokazu Y, Goto M. An entericcoated dry emulsion formulation for oral insulin delivery. Journal of Controlled Release. 2005;107 (1):91-6.

160. Toorisaka E, Ono H, Arimori K, Kamiya N, Goto M. Hypoglycemic effect of surfactant-coated insulin solubilized in a novel solid-in-oil-in-water (S/O/W) emulsion. International Journal of Pharmaceutics. 2003;252 (1-2):271-4.

161. Cilek A, Celebi N, Tirnaksiz F, Tay A. A lecithin-based microemulsion of rhinsulin with aprotinin for oral administration: Investigation of hypoglycemic effects in non-diabetic and STZ-induced diabetic rats. International Journal of Pharmaceutics. 2005;298 (1):176-85.

162. Cilek A, Celebi N, Tirnaksiz F. Lecithin-Based Microemulsion of a Peptide for Oral Administration: Preparation, Characterization, and Physical Stability of the Formulation. Drug Delivery. 2006;13 (1):19-24.

163. Badwan AA, Al-Remawi M, El-Taher T, Elsayed A, inventors; (The Jordanian Pharmaceutical Manufacturing Co., Jordan; Terramark Markencreation $\mathrm{GmbH})$. assignee. Oral delivery of protein drug using microemulsion. Application: WO patent. 200720061103.

164. Zhang J, inventor (Zhang, Hao, Peop. Rep. China). assignee. Oral insulin emulsion or self-microemulsion and capsule with colon-specific release. Application: CN patent 200520040831.

165. Gore MP, inventor (USA). assignee. Oral pharmaceuticals based on jettable microemulsions containing oils and drugs and surfactants and aqueous solutions. Application: US patent 200520040419. 
166. Katayama K, Kato Y, Onishi H, Nagai T, Machida Y. Double Liposomes: Hypoglycemic Effects of Liposomal Insulin on Normal Rats. Drug Development and Industrial Pharmacy. 2003;29 (7):725-31.

167. Degim Z, Uenal N, Essiz D, Abbasoglu U. The effect of various liposome formulations on insulin penetration across Caco-2 cell monolayer. Life Sciences. 2004;75 (23):2819-27.

168. Goto T, Morishita M, Nishimura K, Nakanishi M, Kato A, Ehara J, et al. Novel Mucosal Insulin Delivery Systems Based on Fusogenic Liposomes. Pharmaceutical Research. 2006;23 (2):384-91.

169. Ye S, Wang C, Liu X, Tong Z, Ren B, Zeng F. New loading process and release properties of insulin from polysaccharide microcapsules fabricated through layer-by-layer assembly. Journal of Controlled Release. 2006;112 (1):79-87.

170. Sajeesh S, Sharma CP. Novel $\mathrm{pH}$ responsive polymethacrylic acid-chitosanpolyethylene glycol nanoparticles for oral peptide delivery. Journal of Biomedical Materials Research, Part B: Applied Biomaterials. 2006;76B (2):298-305.

171. Haupt S, Rubinstein A. The colon as a possible target for orally administered peptide and protein drugs. Critical Reviews in Therapeutic Drug Carrier Systems. 2002;19 (6):499-551.

172. Tozaki H, Nishioka J, Komoike J, Okada N, Fujita T, Muranishi S, et al. Enhanced absorption of insulin and $(\operatorname{Asu}(1,7)$ )eel-calcitonin using novel azopolymer-coated pellets for colon-specific drug delivery. Journal of pharmaceutical sciences. 2001;90 (1):89-97.

173. Joseph JW, Kalitsky J, St-Pierre S, Brubaker PL. Oral delivery of glucagonlike peptide-1 in a modified polymer preparation normalizes basal glycemia in diabetic db/db mice. Diabetologia. 2000;43 (10):1319-28.

174. Xia Q, Wang J, Shen W.C. Hypoglycemic Effect of Insulin-Transferrin Conjugate in Streptozotocin-Induced Diabetic Rats; 2000. 295 (2):594-600.

175. Sudhakar Y, Kuotsu K, Bandyopadhyay AK. Buccal bioadhesive drug delivery. Journal of Controlled Release. 2006;114 (1):15-40.

176. Veuillez F, Kalia YN, Jacques Y, Deshusses J, Buri P. Factors and strategies for improving buccal absorption of peptides. European Journal of Pharmaceutics and Biopharmaceutics. 2001;51 (2):93-109. 
177. Morishita M, Barichello JM, Takayama K, Chiba Y, Tokiwa S, Nagai T. Pluronic F-127 gels incorporating highly purified unsaturated fatty acids for buccal delivery of insulin. International Journal of Pharmaceutics. 2001;212 (2):289-93.

178. Guevara-Aguirre J, Guevara-Aguirre M, Saavedra J, Bernstein G, Rosenbloom AL. Comparison of oral insulin spray and subcutaneous regular insulin at mealtime in type 1 diabetes. Diabetes Technology \& Therapeutics. 2007;9 (4):372-6.

179. Bernstein G. The buccal delivery of insulin. Pharm Tech Japan. 2006;22 (2):249-51.

180. Yang T-Z, Wang X-T, Yan X-Y, Zhang Q. Phospholipid deformable vesicles for buccal delivery of insulin. Chemical \& Pharmaceutical Bulletin. 2002;50 (6):749-53.

181. Ishida M, Machida Y, Nambu N, Nagai T. Pharmaceutical interactions in dosage forms and processing. Part XXI. New mucosal dosage form of insulin. Chemical \& Pharmaceutical Bulletin. 1981;29 (3):810-16.

182. Venugopalan P, Sapre A, Venkatesan N, Vyas SP. Pelleted bioadhesive polymeric nanoparticles for buccal delivery of insulin: preparation and characterization. Pharmazie. 2001;56 (3):217-9.

183. Xu H-b, Huang K-x, Zhu Y-s, Gao Q-h, Wu Q-z, Tian W-q, et al. Hypoglycemic effect of a novel insulin buccal formulation on rabbits. Pharmacological Research. 2002;46 (5):459-67.

184. Hinchcliffe M, Illum L. Intranasal insulin delivery and therapy. Advanced Drug Delivery Reviews. 1999;35 (2,3):199-234.

185. Zhang Y, Jiang X-G, Yao J. Nasal absorption enhancement of insulin by sodium deoxycholate in combination with cyclodextrins. Acta Pharmacologica Sinica. 2001;22 (11):1051-6.

186. Pillion DJ, Bartlett JD, Meezan E, Yang M, Crain RJ, Grizzle WE. Systemic absorption of insulin delivered topically to the rat eye. Invest Ophthalmol Vis Sci. 1991 Nov;32 (12):3021-7.

187. Shao Z, Mitra AK. Nasal membrane and intracellular protein and enzyme release by bile salts and bile salt-fatty acid mixed micelles: correlation with facilitated drug transport. Pharmaceutical Research. 1992;9 (9):1184-9. 
188. Soane RJ, Frier M, Perkins AC, Jones NS, Davis SS, Illum L. Evaluation of the clearance characteristics of bioadhesive systems in humans. International Journal of Pharmaceutics. 1999; 178 (1):55-65.

189. Soane RJ, Hinchcliffe M, Davis SS, Illum L. Clearance characteristics of chitosan based formulations in the sheep nasal cavity. International Journal of Pharmaceutics. 2001;217 (1-2):183-91.

190. Varshosaz J, Sadrai H, Heidari A. Nasal Delivery of Insulin Using Bioadhesive Chitosan Gels. Drug Delivery. 2006;13 (1):31-8.

191. Dyer AM, Hinchcliffe M, Watts P, Castile J, Jabbal-Gill I, Nankervis R, et al. Nasal Delivery of Insulin Using Novel Chitosan Based Formulations: A Comparative Study in Two Animal Models Between Simple Chitosan Formulations and Chitosan Nanoparticles. Pharmaceutical Research. 2002;19 (7):998-1008.

192. Varshosaz J, Sadrai H, Alinagari R. Nasal delivery of insulin using chitosan microspheres. J Microencapsul. 2004 Nov;21 (7):761-74.

193. Kwon J-H, Lee B-H, Lee J-J, Kim C-W. Insulin microcrystal suspension as a long-acting formulation for pulmonary delivery. European Journal of Pharmaceutical Sciences. 2004;22 (2-3):107-16.

194. Surendrakumar K, Martyn GP, Hodgers ECM, Jansen M, Blair JA. Sustained release of insulin from sodium hyaluronate based dry powder formulations after pulmonary delivery to beagle dogs. Journal of Controlled Release. 2003;91 (3):385-94.

195. Mitra R, Pezron I, Li Y, Mitra AK. Enhanced pulmonary delivery of insulin by lung lavage fluid and phospholipids. International Journal of Pharmaceutics. 2001;217 (1-2):25-31.

196. Todo H, Okamoto H, Iida K, Danjo K. Effect of additives on insulin absorption from intratracheally administered dry powders in rats. International Journal of Pharmaceutics. 2001;220 (1-2):101-10.

197. Hussain A, Yang T, Zaghloul A-A, Ahsan F. Pulmonary Absorption of Insulin Mediated by Tetradecyl-b-Maltoside and Dimethyl-b-Cyclodextrin. Pharmaceutical Research. 2003;20 (10):1551-7.

198. Kawashima Y, Yamamoto H, Takeuchi H, Fujioka S, Hino T. Pulmonary delivery of insulin with nebulized dl-lactide/glycolide copolymer (PLGA) nanospheres to prolong hypoglycemic effect. Journal of Controlled Release. 1999;62 (1-2):279-87. 
199. Morlock M, Koll H, Winter G, Kissel T. Microencapsulation of rherythropoietin, using biodegradable poly(D, L-lactide-co-glycolide). Protein stability and the effects of stabilizing excipients. European Journal of Pharmaceutics and Biopharmaceutics. 1997;43 (1):29-36.

200. Murillo M, Goni MM, Irache JM, Arangoa MA, Blasco JM, Gamazo C. Modulation of the cellular immune response after oral or subcutaneous immunization with microparticles containing Brucella ovis antigens. Journal of Controlled Release. 2002;85 (1-3):237-46.

201. DeFronzo RA, Bergenstal RM, Cefalu WT, Pullman J, Lerman S, Bode BW, et al. Efficacy of inhaled insulin in patients with type 2 diabetes not controlled with diet and exercise: a 12-week, randomized, comparative trial. Diabetes Care. 2005;28 (8):1922-8.

202. Rosenstock J, Zinman B, Murphy LJ, Clement SC, Moore P, Bowering CK, et al. Inhaled insulin improves glycemic control when substituted for or added to oral combination therapy in type 2 diabetes a randomized, controlled trial. Annals of Internal Medicine. 2005;143 (8):549-58.

203. Skyler JS, Weinstock RS, Raskin P, Yale J-F, Barrett E, Gerich JE, et al. Use of inhaled insulin in a basal/bolus insulin regimen in type 1 diabetic subjects; A 6-month, randomized, comparative trial. Diabetes Care. 2005;28 (7):1630-5.

204. Gale EA. Two cheers for inhaled insulin. Lancet. 2001;357 (9253):324-5.

205. Stoever JA, Palmer JP. Inhaled insulin and insulin antibodies: a new twist to an old debate. Diabetes Technology \& Therapeutics. 2002;4 (2):157-61.

206. Yamamoto A, Luo AM, Dodda-Kashi S, Lee VHL. The ocular route for systemic insulin delivery in the albino rabbit. Journal of Pharmacology and Experimental Therapeutics. 1989;249 (1):249-55.

207. Bartlett JD, Turner-Henson A, Atchison JA, Woolley TW, Pillion DJ. Insulin administration to the eyes of normoglycemic human volunteers. Journal of Ocular Pharmacology. 1994;10 (4):683-90.

208. Soni V, Singh R, Srinivasan R, Jain SK. Pulsatile insulin delivery through the ocular route. Drug Delivery. 1998;5 (1):47-51.

209. Srinivasan R, Jain SK. Insulin delivery through the ocular route. Drug Delivery. 1998;5 (1):53-5.

210. Hosny EA. Relative hypoglycemia of rectal insulin suppositories containing deoxycholic acid, sodium taurocholate, polycarbophil, and their combinations 
in diabetic rabbits. Drug Development and Industrial Pharmacy. 1999;25 (6):745-52.

211. Adikwu MU. Evaluation of snail mucin motifs as rectal absorption enhancer for insulin in non-diabetic rat models. Biological \& Pharmaceutical Bulletin. 2005;28 (9):1801-4.

212. Yun M, Choi H, Jung J, Kim C. Development of a thermo-reversible insulin liquid suppository with bioavailability enhancement. Int J Pharm. 1999 Nov 5;189 (2):137-45.

213. Bertram R, Sherman A, Satin LS. Metabolic and electrical oscillations: partners in controlling pulsatile insulin secretion. American Journal of Physiology. 2007;293 (4, Pt. 1):E890-E900.

214. Steil GM, Panteleon AE, Rebrin K. Closed-loop insulin delivery-the path to physiological glucose control. Adv Drug Deliv Rev. 2004 Feb 10;56 (2):12544.

215. Zhang K, Wu XY. Modulated insulin permeation across a glucose-sensitive polymeric composite membrane. J Control Release. 2002;80 (1-3):169-78.

216. Traitel T, Cohen Y, Kost J. Characterization of glucose-sensitive insulin release systems in simulated in vivo conditions. Biomaterials. 2000;21 (16):1679-87.

217. Burgess DJ, Crommelin DJ, Hussain AS, Chen ML. Assuring quality and performance of sustained and controlled released parenterals. Eur J Pharm Sci. 2004 Apr;21 (5):679-90.

218. Shao PG, Bailey LC. Stabilization of $\mathrm{pH}$-induced degradation of porcine insulin in biodegradable polyester microspheres. Pharm Dev Technol. 1999;4 (4):633-42.

219. Shao PG, Bailey LC. Porcine insulin biodegradable polyester microspheres: stability and in vitro release characteristics. Pharm Dev Technol. $2000 ; 5$ (1):19.

220. Shenoy DB, D'Souza RJ, Tiwari SB, Udupa N. Potential applications of polymeric microsphere suspension as subcutaneous depot for insulin. Drug Dev Ind Pharm. 2003 May;29 (5):555-63.

221. Uchida T, Yagi A, Oda Y, Nakada Y, Goto S. Instability of bovine insulin in poly(lactide-co-glycolide) (PLGA) microspheres. Chem Pharm Bull (Tokyo). 1996 Jan;44 (1):235-6. 
222. Yamaguchi Y, Takenaga M, Kitagawa A, Ogawa Y, Mizushima Y, Igarashi R. Insulin-loaded biodegradable PLGA microcapsules: initial burst release controlled by hydrophilic additives. J Control Release. 2002 Jun 17;81 (3):23549.

223. Kwon YM, Kim SW. Biodegradable triblock copolymer microspheres based on thermosensitive sol-gel transition. Pharm Res. 2004 Feb;21 (2):339-43.

224. Creque HM, Langer R, Folkman J. One month of sustained release of insulin from a polymer implant. Diabetes. 1980 Jan;29 (1):37-40.

225. Davis BK. Control of diabetes with polyacrylamide implants containing insulin. Experientia. 1972 Mar 15;28 (3):348.

226. Li J, Kamath K, Dwivedi C. Gellan film as an implant for insulin delivery. J Biomater Appl. 2001 Apr;15 (4):321-43.

227. Miyazaki S, Yokouchi C, Takada M. External control of drug release: controlled release of insulin from a hydrophilic polymer implant by ultrasound irradiation in diabetic rats. J Pharm Pharmacol. 1988 Oct;40 (10):716-7.

228. Wang PY. Prolonged release of insulin by cholesterol-matrix implant. Diabetes. 1987 Sep;36 (9):1068-72.

229. Wang PY. Palmitic acid as an excipient in implants for sustained release of insulin. Biomaterials. 1991 Jan; 12 (1):57-62.

230. Surini S, Akiyama H, Morishita M, Nagai T, Takayama K. Release phenomena of insulin from an implantable device composed of a polyion complex of chitosan and sodium hyaluronate. J Control Release. 2003 Jul 31;90 (3):291301.

231. Yamakawa I, Kawahara M, Watanabe S, Miyake Y. Sustained release of insulin by double-layered implant using poly(D,L-lactic acid). J Pharm Sci. 1990 Jun;79 (6):505-9.

232. Yamakawa I, Watanabe S, Matsuno Y, Kuzuya M. Controlled release of insulin from plasma-irradiated sandwitch device using poly-DL-lactic acid. Biol Pharm Bull. 1993 Feb;16 (2):182-7.

233. Hall DA, Kadish AH, inventors; (Beckman Instruments, Inc.). assignee. Measurement of glucose concentration in blood. DE patent 1598079. 1972.

234. Srinivasan R, Kadish AH, Sridhar R. Mathematical model for the control mechanism of free fatty acid-glucose metabolism in normal humans. Computers and Biomedical Research. 1970;3 (2):146-65. 
235. Kadish AH. Automation Control of Blood Sugar. I. a Servomechanism for Glucose Monitoring and Control. Am J Med Electron. 1964;3:82-6.

236. Barichello JM, Morishita M, Takayama K, Nagai T. Absorption of insulin from pluronic F-127 gels following subcutaneous administration in rats. Int $\mathrm{J}$ Pharm. 1999 Jul 20;184 (2):189-98.

237. Kim YJ, Choi S, Koh JJ, Lee M, Ko KS, Kim SW. Controlled release of insulin from injectable biodegradable triblock copolymer. Pharm Res. 2001 Apr;18 (4):548-50.

238. Brange J. Stability of insulin - physical and chemical. Dansk Kemi. 1995;76 (8):10-2, 4-5.

239. Jersild M, Hallas-Moller K, Petersen K, Schlichtkrull J. Clinical investigations on new preparations of prolonged action insulin; zinc-insulin preparation for daily injection. Sem Med. 1954;105 (3):110-4.

240. Xiao B, Dodson GG, Dodson EJ, Clarkson J, Turkenburg J, Derewenda U, et al. Mutant insulin crystal packing. Journal of Crystal Growth. 1992;122 (14):144-51.

241. Brange J, Langkjaer L. Chemical stability of insulin. 3. Influence of excipients, formulation, and pH. Acta Pharm Nord. 1992;4 (3):149-58.

242. Harrison L, Kay T, Colman P, Coventry J. In: Turtle J, Osato S, editors. Diabetes in the New Millennium. Sydney: The Endocrinology and Diabetes Research Foundation of the University of Sydney; 1999. p. 85-100.

243. Alberti G, Zimmet P, Shaw J, Bloomgarden Z, Kaufman F, Silink M. Type 2 diabetes in the young: the evolving epidemic: the international diabetes federation consensus workshop. Diabetes Care. 2004 Jul;27 (7):1798-811.

244. Zimmet P, Alberti KG, Shaw J. Global and societal implications of the diabetes epidemic. Nature. 2001 Dec 13;414 (6865):782-7.

245. Uversky VN, Garriques LN, Millett IS, Frokjaer S, Brange J, Doniach S, et al. Prediction of the association state of insulin using spectral parameters. Journal of Pharmaceutical Sciences. 2003;92 (4):847-58.

246. Lakeshore Biomaterials, http://www.lakeshorebiomaterials.com/productsstock-polymers.html, Last accessed: October 25th, 2007.

247. Choi S, Kim SW. Controlled release of insulin from injectable biodegradable triblock copolymer depot in ZDF rats. Pharm Res. 2003 Dec;20 (12):2008-10. 
248. Saemann A, Muehlhauser I, Bender R, Hunger-Dathe W, Kloos C, Mueller UA. Flexible intensive insulin therapy in adults with type 1 diabetes and high risk for severe hypoglycemia and diabetic ketoacidosis. Diabetes Care. 2006;29 (10):2196-9.

249. Wang F, Carabino JM, Vergara CM. Insulin glargine: a systematic review of a long-acting insulin analogue. Clin Ther. 2003 Jun;25 (6):1541-77, discussion $39-40$.

250. Home PD, Ashwell SG. An overview of insulin glargine. Diabetes/Metabolism Research and Reviews. 2002;18 (Suppl. 3):S57-S63.

251. Owens DR, Griffiths S. Insulin glargine (Lantus). International Journal of Clinical Practice. 2002;56 (6):460-6. 


\section{VITA}

Mr. Om Anand, was born on 10 June 1976, in a village near Delhi, India. He was admitted to the College of Pharmacy, Delhi University, Delhi in August 1993. Mr. Om Anand graduated with a Bachelor of Pharmacy degree from Delhi University in July 1997. He was admitted to the Department of Pharmaceutical Sciences, Punjabi

University, Patiala, Punjab, India in September 1997 and graduated with a Masters in Pharmaceutics degree in February 2000.

He joined Ranbaxy Research Laboratories as a Research Associate in March 2000, where he worked in the Department of Novel Drug Delivery Systems (NDDS) for formulation development of ANDAs and NDAs.

Mr. Om Anand was enrolled in the Doctor of Philosophy program at the University of Tennessee Health Science Center in August 2003 and received a Graduate Teaching Assistantship. He joined Dr. Atul J. Shukla's group in Department of Pharmaceutical Sciences in November 2004.

Mr. Om Anand is a member of the Rho Chi Pharmaceutical Honor Society, the American Association of Pharmaceutical Scientists and the Controlled Release Society. 